\title{
Mental Models of Behavior Change: Can Mindfulness and Spirituality support Sustainable Food Consumption?
}

\author{
Dissertation \\ to attain the doctoral degree (Dr. sc. agr.) \\ at the Faculty of Agricultural Sciences, \\ Georg-August-University Goettingen, Germany
}

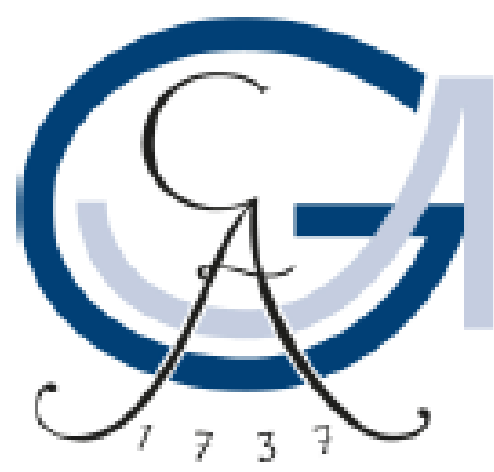

Submitted by

Aspasia Werner

Born in Leonberg, Germany

August 2020 
Supervision and examination committee

First supervisor: $\quad$ Prof. Dr. Achim Spiller

Second supervisor: $\quad$ Prof. Dr. Yasemin Boztuğ

Third supervisor: $\quad$ Prof. Dr. Meike Wollni

Date of submission: $\quad 10.08 .2020$

Date of defense: $\quad 08.10 .2020$ 
"Emancipate yourself from mental slavery

none but ourselves can free our mind.

$$
\text { (...) }
$$

Won't you help to sing

these songs of freedom?"

Bob Marley; Redemption Song 


\section{Table of Contents}

Table of Contents ___ iv

Acknowledgements___ vi

List of Abbreviations ___ vii

Glossary of Pali Terms___ viii

List of Tables ___ ix

List of Figures___ xi

Chapter 1 General Introduction ___ 1

1.1 Food Consumption and Sustainability _____ 1

1.2 Mental Models, Shifting Paradigms, and the rise of Spiritual Values in the Western Society ___ 2

1.3 The Role of Mindfulness in the Construction of Mental Models __ 4

1.4 Common Theories of Consumption Behavior ___ 5

1.5 Study Aims and Guiding Questions ___ 7

1.6 Summaries of the three Essays_____ 8

1.6.1 Essay 1: What it takes to Change: Adoption and Maintenance of Alternative dietary Lifestyles

1.6.2 Essay 2: The Yoga of Sustainable Diets: Exploring Consumers Mind and Spirit.__ 9

1.6.3 Essay 3: Towards a new Approach of addressing Mindfulness into Sustainable Food Consumption Behaviors 10

1.7 General Conclusion _______________ 11

1.7.1 Tying up loose Ends: Thoughts and Implications ___ 12

1.7.2 Limitation of the presented Research___ 16

Chapter 2 What it takes to change: Adoption and Maintenance of Alternative dietary Lifestyles 19

2.1 Introduction ___ 19

2.2 Methods 22

2.2.1 Activity-based in-depth Interviews __ 22

2.2.2 Participants 24

2.2.3 Qualitative Content Analysis___ 25

2.2.4 Analysis of activity based Material __ 25

2.2.5 Sample Description __ 26

2.3 Results _____________________ 29

2.3.1 General Motivations for dietary Lifestyle Changes _ 29

2.3.2 Mentalities of pertinent Dietary Changes___ 30

$\mathbf{2 . 4}$ Discussion and Conclusion ___ 34

Chapter 3 The Yoga of Sustainable Diets: Exploring Consumers Mind and Spirit __ 39

3.1 Introduction __ 40

3.2 Literature research ___ 41

3.2.1 Theoretical Considerations __ 41 
3.2.2 The Role of Spirituality in Food Choice and Diet ___ 43

3.2.3 The Role of Mindfulness in Food Choice and Diet __ 46

3.2.4 Study Aims _ـ 48

3.3 Methods 40

3.3.1 The Questionnaire __ 49

3.3.2 Data Analysis __

3.4 Results ________________ 53

3.4.1 Sample Description _ 53

3.4.2 Factor Structures derived from the EFA 54

3.4.3 The Effect of Spirituality and Mindfulness on Food Choice and Diet __ 59

3.5 Discussion _______ 63

3.5.1 The Value of Spirituality and Mindfulness for Sustainable Food Consumption Research _ 63

3.5.2 Effects of SPIRIT I, SPIRIT II, MIND I, and MIND II on Consumer Food Choice and Diet __ 65

3.6 Conclusion _______________ 68

3.7 Additional Tables _____________ 69

Chapter 4 Towards a new Approach of addressing mindfulness in Sustainable Food

Consumption Behaviors

4.1 Introduction

4.2 Current Mindfulness Approaches applied to addressing Food Choice and Dietary

change Behavior of Consumers

4.2.1 What is Mindfulness? A Western Approach of an Eastern Concept 74

4.2.2 Western Mindfulness as a Catalyst for stimulating sustainability-relevant Food Consumption Behaviors $\quad 76$

4.2.3 Commonly used Mindfulness Scales for exploring Food Choice and Diet___ 77

4.2.4 Inconsistencies in the Mindfulness-Sustainability Relationship: between Theory and Empirical Research

4.3 Towards a Holistic understanding of Mindfulness: reintroducing Eastern Concepts_83

4.3.1 The Eastern Concept of Mindfulness __ 83

4.3.2 Why it is Important to cultivate Mindfulness skillfully___ 86

4.3.3 Eastern Mindfulness and its Connection to Buddhist Spirituality __ 90

4.3.4 The Role of Ethics and Morality in the Cultivation of Right Mindfulness __ 91

\subsection{Towards an integrated Understanding and Assessment of Mindfulness in}

Sustainable Food Consumption _________________________ 93

4.4.1 Conceptualization and Integration of Mindfulness into the Sustainability Framework _ 94

4.4.2 Important Terms and Definitions__ 96

4.4.3 Implications for developing a Scale to assess Mindfulness __ 101

4.5 Final Remarks ___________ 103

Appendices _ـ 105

Bibliography _ 136

Authors Contributions___ 154

Declaration ___ 155 


\section{Acknowledgements}

The financial support received from the German Research Foundation (DFG) and the Namaste+ program for implementing this research is gratefully acknowledged.

I want to express gratitude to my mentor Prof. Dr. Achim Spiller. Thank you for guiding me through the experience of being a PhD student. Prof. Dr. Yasemin Boztuğ, thank you for having taken on the role as a second referee. Furthermore, I am grateful for the third referee Prof. Dr. Meike Wollni.

I want to thank all members of GlobalFood for enriching my professional life throughout these past three years. Each of you has been a source of inspiration for me. Furthermore, being affiliated with the Chair of Food Marketing, I had the chance to collaborate with great individuals, amongst them I want to express special thanks to my supervisor Dr. Antje Risius. Being able to witness your sharp mind at work impressed and motivated me every time we met and I am deeply grateful for this.

Furthermore, I want to thank Prof. Dr. Mahesh Deokar, Prof. Dr. Rupert Gethin, and the Department of Pali and Buddhist Thought at the Savitribai Phule Pune University for hosting me and supporting my studies.

Few words are able to express the gratitude I feel towards my family members, house mates, friends, and companions. How lucky I am having you in my life! Thank you for the various moments where you supported me without even being aware of how significant your presence was.

"If you have never wept bitter tears because a wonderful story has come to an end and

you must take your leave of the characters with whom you have shared so many adventures, whom you have loved and admired, for whom you have hoped and feared, and without whose company life seems empty and meaningless.

If such things have not been part of your own experience, you probably won't understand what Bastian did next." 


\section{List of Abbreviations}

\begin{tabular}{ll} 
ABC & Attitude-Behavior-Context \\
APF & Animal-Protein based Food \\
BC & Before Christ \\
CD & Convenience Diet \\
CHIME & Comprehensive Inventory of Mindfulness Experiences \\
CSA & Community Supported Agriculture \\
DA & Dietary Awareness \\
EFA & Exploratory Factor Analysis \\
EUR & Euro \\
FFMQ & Five Facet Mindfulness Questionnaire \\
FV & Fruits and Vegetables \\
IFC & Informed Food Choice \\
INR & Indian Rupee \\
MAAS & Mindful Attention Awareness Scale \\
MAR & Missing At Random \\
MBSR & Mindfulness Based Stress Reduction \\
MBCT & Mindfulness Based Cognitive Therapy \\
MCAR & Missing Completely At Random \\
MICE & Multiple Imputation by Chained Equations \\
MIND I & Affirmative mind \\
MIND II & Scattered mind \\
N & Number \\
PCE & Perceived Consumer Effectiveness \\
PEB & Pro-Environmental Behavior \\
PEBS & Pro-Environmental Behavior Scale \\
PPF & Plant-Protein based Food \\
SAIL & Spiritual Attitude and Involvement List \\
SDG & Sustainable Development Goals \\
SPIRIT I & Supernatural spirituality \\
SPIRIT II & Natural spirituality \\
TCM & Traditional Chinese Medicine \\
TEMS & The Eating Motivation Survey \\
USA & United States of America \\
USD & United States-Dollar \\
VBN & Value-Belief-Norm \\
VIVO & Vital Impact Assessment Scale \\
& \\
\hline
\end{tabular}




\section{Glossary of Pali Terms}

Ariyo atthangiko maggo

The Buddhist Noble Eight-factored Path

Dhamma law, teaching, ultimate constituent of reality

Jhâna

Saññā

State of absorption in meditation

Sati

Apperception

Satipaţ̧hāna

Mindfulness

Establishing of mindfulness 


\section{List of Tables}

Table 2.1: The demographic profile of the 17 interviewees participating in the alternative dietary lifestyle motivation study. Data were collected through activity-based, in-depth interviews in Germany from July to October 2019.

Table 2.2: Overview of the main dietary characteristics for each type of diet sampled throughout the survey from August to October 2019. 28

Table 3.1: The summary chart of the socio-demographic profile of the respondents from India and the United States of America (USA) in spring 2018. Per annum income level: India: high $=$ more than $1,200.000 \mathrm{INR}$, middle $=120.000-1,200.000 \mathrm{INR}$, low $=$ less than 120.000 INR; USA: high= more than 100.000 USD, middle $=35.000-100.000$ USD, low = less than 35.000 USD; $1,000,00 €$ equal $80.654,00$ INR equal 1,174,11 USD, based on the official course rate (09.07.2018); education level: India: high = master's and higher, medium = senior school and bachelor's, low = less than senior school, USA: high = master's and higher, medium = associate's and bachelor's, low = less than college. 54

Table 3.2: Summary of the results of the two Exploratory Factor Analyses performed for selfreported food choice and dietary behavior $(n=1012)$ and trait spirituality and trait mindfulness. Data was collected through online surveys from India and the United States in spring 2018. 55

Table 3.3: List of the item loadings of the four factors (SPIRIT I, SPIRIT II, MIND I, and MIND II) derived during EFA procedure for trait spirituality and trait mindfulness based on 1012 observations. Data was collected through online surveys from India and the United States in spring 2018. 57

Table 3.4: Regression results for various food choice and dietary behavior factors in dependence to trait spirituality and trait mindfulness factors (SPIRIT I, SPIRIT II, MIND I, and MIND II) and four socio-demographic traits (age, gender, education, and income) of Indian consumers. Level of significance at ${ }^{*} p<0.05$, ${ }^{* *} p<0.01$, and ${ }^{* * *} p<0.001$. This table does not include all traits considered within the analysis. Detailed tables, including the socio-demographic measures are given in chapter 3.7. 60

Table 3.5: Regression results for various food choice and dietary behavior factors in dependence to trait spirituality and trait mindfulness factors (SPIRIT I, SPIRIT II, MIND I, and MIND II) and four socio-demographic traits (age, gender, education, and income) of US consumers. Level of significance at ${ }^{*} p<0.05$, ${ }^{* *} p<0.01$, and ${ }^{* * *} p<0.001$. This table does not include all traits considered within the analysis. Detailed tables, including the socio-demographic measures are given in chapter 3.7 . 60

Table 3.6: Regression results for various food choice and dietary behavior factors in dependence to trait spirituality and trait mindfulness factors (SPIRIT I, SPIRIT II, MIND I, and MIND II) and four socio-demographic traits (age, gender, education, and income) of Indian consumers. Level of significance at ${ }^{*} p<0.05,{ }^{* *} p<0.01$, and ${ }^{* \star *} p<0.001$. 69 
Table 3.7: Regression results for various food choice and dietary behavior factors in dependence to trait spirituality and trait mindfulness factors (SPIRIT I, SPIRIT II, MIND I, and MIND II) and four socio-demographic traits (age, gender, education, and income) of US-American consumers. Level of significance at ${ }^{*} p<0.05,{ }^{* *} p<0.01$, and ${ }^{* * *} p<0.001$ 70 


\section{List of Figures}

Figure 1.1: A simplified model of the mental processes involved in motivating behavioral response.

Straight line = direct effect; dashed line $=$ indirect effect

Figure 1.2: The influence of mindfulness on the mental processes involved in motivating behavioral response. Straight line $=$ direct effect; dashed line $=$ indirect effect.

Figure 1.3: The Alphabet Theory for explaining food purchase behavior according to ZEPEDA AND DEAL 2009. Straight line = direct effect; dashed line = indirect effect.

Figure 2.1: The sheet provided for activity 2. The 30 statement stickers, derived from the VIVO questionnaire, were arranged in a Lickert-scale manner based on the correctness of the statement for the participants' characters.

Figure 2.2: The three recurring patterns found when transitioning to a new dietary pattern. Results are based on interview data collected from participants following different alternative dietary lifestyles.

Figure 4.1: The integrative brain model for behavior change through mindfulness, taken and adapted from Tang and colleagues (2015).

Figure 4.2: The four essential functions of mindfulness according to Kuan 2008 (modified). 85

Figure 4.3: The elements of the Buddhist Noble Eight-factored Path to liberation 87

Figure 4.4: The position of right mindfulness in the process of mental liberation. 89

Figure 4.5: The position of right mindfulness within the process of behavior formation. 94

Figure 4.6: A proposed theoretical model for measuring right mindfulness in sustainability sciences. 102 


\section{Chapter 1 General Introduction}

This dissertation explores the relationship between sustainable food consumerism and mental models. The following subchapters provide a general overview on the topic by providing a problem statement, the general theoretical background, as well as summaries of the three essays presented in this dissertation. Furthermore, based on the knowledge acquired through the essays general implications are discussed at the end of this chapter.

\subsection{Food Consumption and Sustainability}

During the last century global food habits have changed, resulting in considerable increases in the consumption of animal protein, saturated fat and sugar (ATKINS AND BOWLER 2001, Broglia AND KAPEL 2011). This nutrition transition, also known as westernization of diets, is a result of rising economic welfare, global trade, technology, and increasing urbanization (BELAHSEN 2014). Since this development adversely affects the environment, the society, and the economy, issues of how to regulate and navigate food consumption behavior towards a reduced negative eco-social impact, have, therefore, received considerable attention within the national and international community (REISCH et al. 2013). However, modern consumption societies are characterized by personal comfort, convenience, and freedom of choice (LIM 2017). This poses additional challenges to the adoption of sustainable food consumption practices as they are largely navigated through habit and automation (SPAARGAREN et al. 2013). The challenge of shifting food consumption behaviors towards greater sustainability, therefore, needs to be addressed through an extended framework of strategic guidance. While political tools of the past tended to rationalize consumerism, nowadays more and more emphasis is placed on the intrinsic motivational and mental aspects stimulating positive consumption behavior change (SORON 2010). The concepts of worldview (VAN EGMOND AND DE VRIES 2011, HeDLUND-DE WITT et al. 2014) and mindfulness (FISCHER et al. 2017) are considered to have a significant effect on the sustainability of consumption behaviors since they build the foundations of human sense-making, thought, and action. Despite their promising implications for sustainable development, however, research on these concepts is still relatively novel and requires further 
investigation in order to draw a clearer picture on their magnitude of effect on sustainable food consumerism.

\subsection{Mental Models, Shifting Paradigms, and the rise of Spiritual Values in the Western Society}

The human mind is designed for efficiency (DEMETRIOU AND SPANOUDIS 2018). Therefore, high amounts of internal and external stimuli are translated into coherent frames of reference or mental models (TENENBAUM et al. 2011). These mental models can be defined as selective toolkits motivating behavior (HEMFORTH AND KONIECZNY 2006). Figure 1.1 shows a simplified model of the mental structures involved with information processing.

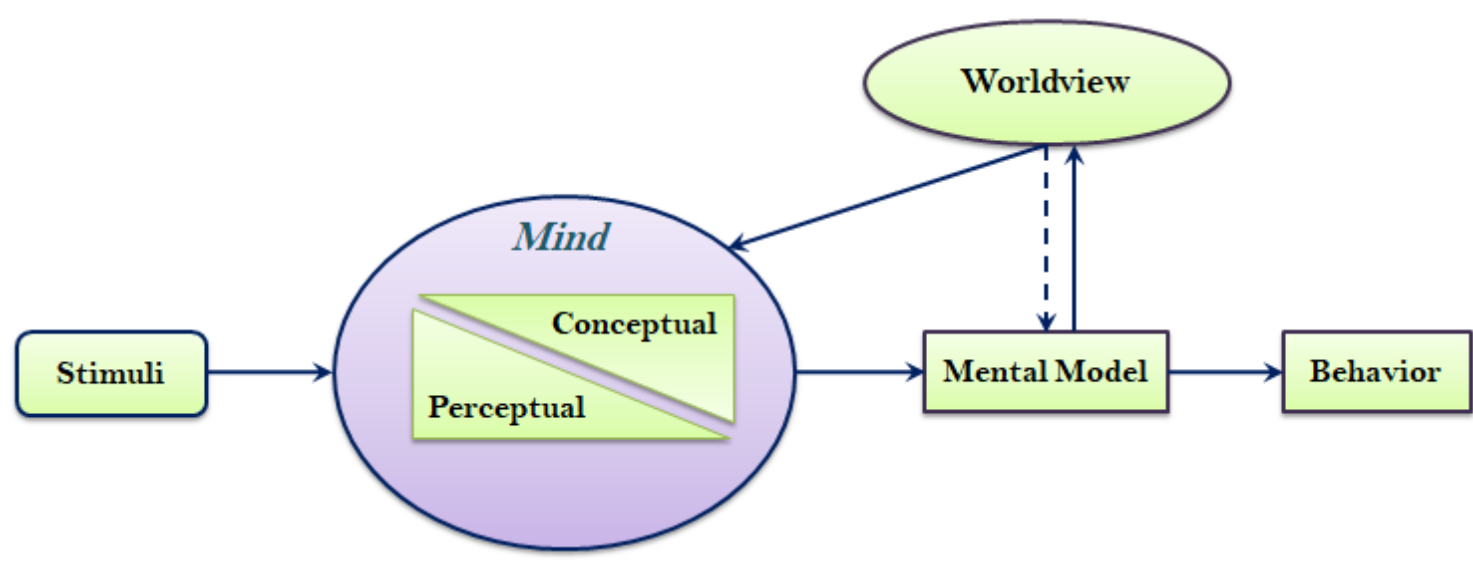

Figure 1.1: A simplified model of the mental processes involved in motivating behavioral response. Straight line $=$ direct effect; dashed line $=$ indirect effect.

For the processing of external and internal stimuli, the mind operates in two modes: (1) perceptual and (2) conceptual (WILLIAMS 2010). The first mode refers to the sensory-based processing of stimuli including vision, scent, sound, taste, and touch. The second mode is more verbally inclined. It comprises mental processes like thought, memory, judgment, analysis, rumination, planning, and comparison. Through this, incoming stimuli are processed based on subjective experience and knowledge (WERHANE et al. 2011). To navigate through everyday life, humans are heavily dependent on the conceptual mental mode as it enables them to filter and translate incoming information into simplified mental models of the real world (WILLIAMS 2010). The totality of mental models applied to understand reality is considered as a person's 
worldview (BALDWIN AND BLOM 2010). It is the overarching concept linking mental models. The worldview, in turn, indirectly reinforces specific mental models as it guides the minds' attention towards collecting concurrent evidence. However, due to the subjectivity of personal experience, mental models are considered incomplete and biased representation of the world (WERHANE et al. 2011). Hence, mental models do not necessarily reflect reality but rather one's personal perception of reality. Based on the active mental models, response behavior is stimulated.

Mental models are considered prone to habit and rather difficult to change (BALDWIN AND BLOM 2010). However, change may still be possible as the perceptual mode of the mind constantly evaluates if incoming stimuli match with the established mental models. Changes in mental models can then be induced through cognitive dissonance (OLSEN et al 1992, WERHANE et al. 2011). Cognitive dissonance can get triggered by internal or external stimuli (OLSEN et al 1992). The former describes an experience-based personal insight. It occurs in cases where enough information to reject the formerly held mental model has accumulated. The latter occurs when commonly shared worldviews don't match real conditions.

Even though worldviews may vary greatly amongst individuals, there are notable trends that are shared by communities, societies, and cultures (AERTS et al. 1994). In fact, without these communalities the development of social structures and collective problem resolution would be difficult (KLEIN 2017). During past decades, however, significant changes in human worldview have been noticed especially within Western societies (TISCHLER 1999, HUSEMANN AND ECKHARDT 2019). BOURNE (2009) characterizes the main elements of this paradigm shift as an increasing believe in: (1) a conscious and coherent universe, (2) a multidimensional reality, (3) a collective consciousness, (4) the complementarity of sciences and spirituality, (5) intuitive and visionary forms of knowledge, (6) a consciousness that has a causal effect, and (7) natural ethics based on authenticity rather than cultural norms. In sum, these elements point to a rise in non-denominational spiritual values amongst the members of society. 
With growing scientific interest, the exploration of the relationship between spirituality and consumerism grew (e.g. BALL et al. 2001, REDDEN 2016). These research encounters mainly focus on the exploration of consumers religiousness and spirituality (e.g. ARNOULD et al. 1999, MARMOR-LAVIE AND Stout 2016), spiritual branding (e.g. HeElas et al. 2005, RinAlLO et al. 2013), and on spiritual trends in consumerism (e.g. O'GUINN AND BELK 1989, RAHMAN 2012, Buchanan-Olivier AND SCHAU 2013, JOSHI 2017). Furthermore, various scholars agree that this paradigm shift is an important driver of sustainable development (e.g. Schumacher 1995, OrR 2002, HedLund-de Witt 2013, De LA SienRa SERVIN et al. 2017). However, so far, little research has focused on the intrinsic relationship between spiritual values and sustainable food consumption or food lifestyle change. As a result of this, spirituality and spirituality-related themes are still under-represented in public debates around sustainable development (VER BEEK 2000).

\subsection{The Role of Mindfulness in the Construction of Mental Models}

Mindfulness is commonly defined as a state of mind that is aware of the present moment, including everything that happens within the individual as well as the surrounding environment (ERICSON et al. 2014). A detailed background on the history, development, and conceptualization of mindfulness for sustainability sciences will be given in essays two and three. This subchapter focuses on explaining the relationship between mindfulness and mental processing.

The relevance of mindfulness in respect to the concept of mental models stems from its ability to alter mental functioning (VAGO et al. 2018). As shown in figure 1.2, mindfulness is assumed to affect and balance the processing of external stimuli and the interplay between the perceptual and conceptual mental modes. Furthermore, it enables to realize the moment when the mind switches between these two modes (WILLIAMS 2010). Through this, greater awareness of situational experience is generated (WILLIAMS 2010) which affects all following mental processes, resulting in new means of behavior. 


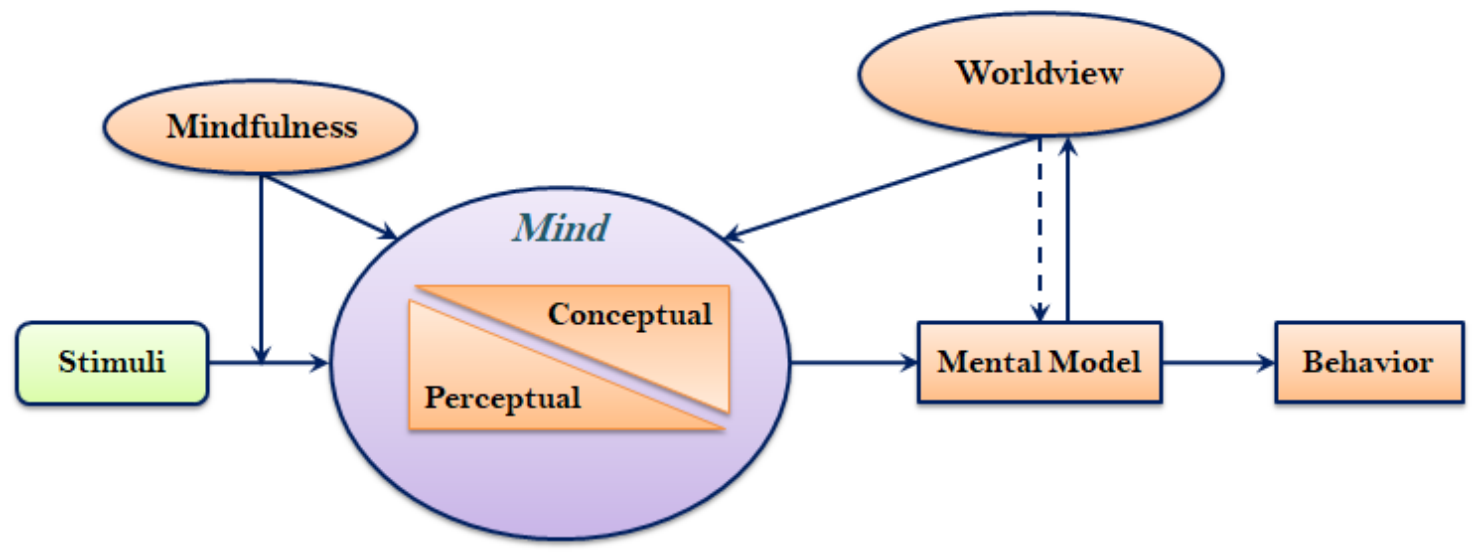

Figure 1.2: The influence of mindfulness on the mental processes involved in motivating behavioral response. Straight line $=$ direct effect; dashed line $=$ indirect effect.

Therefore, the concept of mindfulness may be seen as agent for sustainable behavior with various positive implications on the human psyche. As discussed in more detail in essays one and three, mindfulness is considered to increase altruism and pro-environmental behavior and to reduce materialism and egoism (ROSENBERG 2004, FISCHER et al. 2017). Furthermore, mindfulness can play a crucial role in disrupting habitual behavior also in regard to food consumption (FISCHER et al. 2017).

\subsection{Common Theories of Consumption Behavior}

Consumer sciences moved away from the, until then, common assumption of consumers behaving as rational units that make choices based on their individual utility maximization (SIMON 1955). Especially, STERN's Value-BeliefNorm (VBN) theory (2000) was frequently utilized to explain proenvironmentalism. This theory suggests that pro-environmental behavior is stimulated by three core values which are of either: (1) altruistic, (2) egoistic, or (3) biospheric origin. Altruistic values (1) are oriented towards societal wellbeing whereas egoistic values (2) are based on individual well-being. Issues regarding environmental well-being are called biospheric values (3). According to STERN's implications, individuals are likely to take responsible actions if their values are endangered to be violated. 
In regard to food consumption behavior, important scientific theories include the Attitude-Behavior-Context (ABC) theory (GUAGNANO et al. 1995). According to this theory, food consumption behavior is a context-dependent interactive process based on personal attitudes. This means, that personal attitudes only navigate behavior if the surrounding context, e.g. the financial or social condition, is neutral. However, food consumption behavior is strongly driven by routine which imposes an inherent resistance to change even if surrounding conditions prove neutral (SPAARGAREN et al. 2013). For this reason, ZEPEDA AND DEAL (2009) introduced an extended framework to the ABC theory. Their Alphabet theory aims at explaining food purchase behavior. Its explanatory fundament is built upon the VBN theory, ABC theory, and four additional factors, namely: (1) habit, (2) knowledge, (3) information seeking, and (4) demographic data (see figure 1.3)

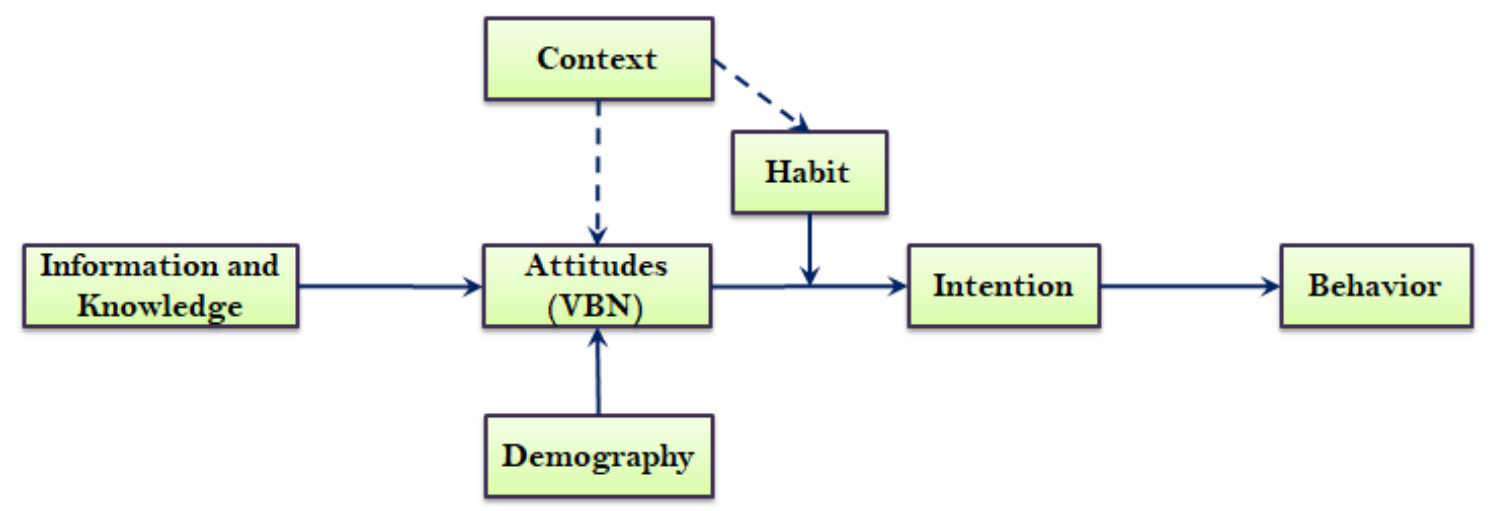

Figure 1.3: The Alphabet Theory for explaining food purchase behavior according to ZEPEDA AND DEAL 2009. Straight line = direct effect; dashed line = indirect effect.

This theory proposes that attitudes are formed through VBN, demography, as well as through knowledge and information seeking. The translation of attitudes into context dependent behavior is mainly mediated through habit. In fact, habits play a key role for expressing food choice and dietary behavior, especially, when external conditions are stable (ZePEDA AND DEAL 2009, SaINSBURY et al. 2018). The extent to which habit influences intention and hence behavior, is determined by the degree of behavioral mastery and the perceived control over the performed behavior (HALL AND FONG 2007). This means, if there are no changes in contextual parameters as well as a sufficient amount of perceived mastery and control over food choice and diet, changes in behavior may only be 
initiated through enforced changes in habitual behavior (SAINSBURY et al. 2018) or intrinsic motivational drivers (VermeIeR AND VerbeKe 2006). In this regard mental models can play a crucial role for guiding food consumption behavior change as they are closely connected to motivation (KLEIN 2017).

\subsection{Study Aims and Guiding Questions}

Different scholastic opinions on how to achieve sustainable food consumerism exist. The presented dissertation explores the role of spiritual mental models and mind techniques for promoting long-lasting sustainable transformation within the society. In order to be more efficient at addressing issues of unsustainable consumerism, a thorough understanding of the mental structures undermining decision making must be established. This dissertation therefore aims at exploring the mental models motivating sustainable food choice and dietary lifestyle. A special focus is placed on the concepts of spirituality and mindfulness as they inform and alter the mental processing of stimuli. The guiding study questions are:

$\infty$ Which mental models and intrinsic motivations lead to permanent shifts in dietary lifestyles?

$\infty$ What are the effects of traits spirituality and mindfulness on food choice and dietary behavior?

$\infty$ How can mental development techniques such as mindfulness, be utilized more efficiently in sustainable consumer sciences?

The presented essays approach mental models and their potential as sustainability agents through diverse means and perspectives. Accordingly, essay one qualitatively explores key mental models motivating strongly alternative dietary lifestyles. Essay two quantifies the effect of the traits spirituality and mindfulness on selected food choice and dietary behaviors. Lastly, essay three extends on the insights gained in essay two by presenting a conceptual suggestion for more refined conceptualization and measurement of mindfulness to better fit the specifics of sustainable consumer sciences. A summary of the three essays is presented in the proceeding subchapter. 


\subsection{Summaries of the three Essays}

\subsubsection{Essay 1: What it takes to Change: Adoption and Maintenance of Alternative dietary Lifestyles}

The first essay draws a clearer picture of the motivational drivers of food lifestyle changes in Germany by investigating why consumers adopt and maintain extreme alternative diets. Qualitative interviews were conducted with 17 participants of whom twelve were female, four were male and one was diverse gendered. The average age was 36.6 years. Furthermore, all participants had received at least upper secondary education. The mean duration of following the corresponding alternative food lifestyle was 7.6 years. In total, eight different alternative dietary lifestyles were represented. These were: (1) Veganism, (2) macrobiotic diet, (3) Five Elements diet, (4) Freeganism, (5) intuitive diet, (6) Anthroposophic diet, (7) Yogic diet, and (8) Chrono diet. Reasons for the adoption and continuation of the respective alternative diets as well as perceived challenges were explored through semistructured, activity-based interviews. Data analysis revealed three factors to be most relevant in motivating the adoption of an alternative dietary lifestyle. These were: (1) key moment, (2) knowledge aquiration, and (3) health concern. The first factor, key moment, represents a sudden insight that changed the mindset of the interviewee. The knowledge factor (2) is based on the accumulation of information on an issue over time which motivated the respondent to shift dietary behavior. The last factor, health concern (3), comprised health issues and/or health concerns. The maintenance of the alternative dietary lifestyles was predominatly guided by three mindsets: (1) the self-reflective mind, (2) the responsible mind, and (3) the interconnected mind. The self-reflective mind (1) comprised an elevated physical and mental well-being and a feeling of being more authentic due to greater self-reflection, self-awareness, and selfresponsibility. In regard to the responsible mindset (2) respondents were driven by a strong sense of responsibility towards others or the environment. The interconnected mind (3) was involved with the feeling of community or communion with others, nature, and/or above. Over all, these findings point to the multidimensional nature of the mental models underpinning alternative food lifestyle choices. They provide valuable suggestions on how to support 
consumers in shifting dietary choices as they draw a clearer picture of the inspirations and obstacles of maintaining an alternative dietary lifestyle. In this regard, self-reflection, social support and inclusion revealed to be of importance for the long-term adoption of alternative food lifestyles. Personality development, public awareness, and acceptance seem to be crucial in order to move towards greater sustainability in eating behavior.

\subsubsection{Essay 2: The Yoga of Sustainable Diets: Exploring Consumers Mind and Spirit.}

The second essay represents a quantitative approach to estimate the influence of new age worldviews on food consumerism. Since spiritual values and mental models are a crucial characteristic of the new paradigm, trait "spirituality" and trait "mindfulness" were chosen to predict changes in food choice and dietary intake. The concrete study questions are: (1) Are there factorial interactions between the spirituality trait and trait mindfulness? (2) What is the effect of these factorial interactions on consumer food choice and dietary behavior? Data was collected through the means of an online self-report questionnaire. In order to account for cultural, economic, and ecological differences, population samples were drawn from India and the USA. A total number of 1.243 questionnaires were collected of which 1.012 observations (482 from India and 530 from the USA) qualified for further data analysis. The analysis process comprised imputation modeling, exploratory factor analysis, and multiple regression analysis. In opposition to the factor analysis, which was performed on the combined data set, imputations were carried out separately for each country. Through this, the calculation of missing data of each data set was based on country specific parameters before the data sets were utilized in the exploratory factor analysis. The factor analysis resulted in four applicable factor themes. These are: (1) supernatural spirituality, (2) natural spirituality, (3) affirmative mindset and (4) scattered mindset. The first factor, supernatural spirituality (1) is closely related to religious beliefs or a more general belief in higher forces and deities. Natural spirituality (2) is centered on the experience of spirituality in relation to the environment and society. An affirmative mindset (3) was expressed through items that referred to a person's ability to be compassionate, supportive, and self-aware while the last factor, scattered 
mindset (4) was found to be inattentive and judgmental. Like the imputations, regression analysis was conducted separately for each country to learn how the factor model translates different socio-cultural and environmental influences. However, certain similarities were noticed when comparing the country specific results with each other. Especially, the latent variables natural spirituality and affirmative mindset revealed to be strong predictors for some of the investigated food consumption variables. Natural spirituality significantly increased fruits and vegetable consumption and decreased the consumption of convenience foods. An affirmative mindset was significantly related to increase proenvironmentalism and dietary awareness. Furthermore, a scattered mindset increased the consumption of convenience foods in both country data sets significantly. The predictive qualities of the variable supernatural spirituality were mostly marginal. In opposition to the Indian dataset, the variable natural spirituality reflected comparably stronger on food choice behavior as compared to the dietary variables. Over all, in regard to factorial relations of trait mindfulness and trait spirituality, this study confirms the independence of both constructs. At the same time an adjusted perspective on important subthemes within the constructs is provided. These subthemes draw a clearer picture on the aspects of traits spirituality and mindfulness that prove most effective in navigating food choice and dietary behaviors like dietary awareness, proenvironmentalism, and fruit and vegetable consumption. However, inconsistencies in regard to the expected effect on certain sustainability measures (e.g. animal protein based diet) were noticed. Further research would therefore benefit from a deeper scientific exploration of the spirituality and mindfulness traits and sustainable food consumerism.

\subsubsection{Essay 3: Towards a new Approach of addressing Mindfulness into Sustainable Food Consumption Behaviors}

In sustainable consumer sciences, the existing accounts conceptualizing and assessing mindfulness fail to resolve the contradiction between theoretical implications and behavioral outcome. While theories suggest that mindful consumers behave more sustainable (e.g. shopping, diet, waste disposal) studies evaluating these relationships show mixed results. Essay three, therefore, addresses the question of how to optimize currently employed 
mindfulness concepts and scales within sustainable consumer sciences. In regard to conceptualization, this essay draws on additional aspects of traditional Indian Buddhism and introduces the term "right" mindfulness and the four essential functions of mindfulness. Mindfulness is defined as "full awareness" comprising the four essential functions: (1) simple awareness, (2) protective awareness, (3) introspective awareness, and (4) deliberately forming conceptions. Through this, the currently applied Western concept of mindfulness get enriched by aspects of wisdom based ethics and moral frameworks which are also considered important as drivers of sustainable consumption. Furthermore, terms and definitions that are frequently used in respect with the concept of mindfulness are clarified, including, amongst others, attention, awareness, non-judgment, and non-reactivity. Based on this more complete understanding of mindfulness, its potential and applicability as sustainability agent is demonstrated. The essay closes by presenting points of improvement in mindfulness scale development especially in regard to selfreport questionnaires. Implications on the interpretation of the commonly utilized mindfulness scales within sustainable consumer sciences are provided. In regard to the development at scale the utilization of methods that stimulate respondents on various levels of cognition are suggested. Since mindfulness is connected to the skillful navigation of emotions and feelings, using visual tools, triggering different mental structures, may prove helpful for evaluating mindfulness. Moreover, the introduction of different response modes like open questions or buzzwords is suggested as well. Through this, essay three provides valuable points of consideration for the further development and refinement of current mindfulness definitions and scales used within sustainable consumer sciences.

\subsection{General Conclusion}

Starting in the second half of the twentieth century, a new, more spiritually inclined, worldview emerged that has expanded ever since (CAPRA 1983, BOURNE 2009). However, until recently, spiritual values and mind development techniques have received little attention within the field of sustainability sciences. The presented dissertation is one amongst very few looking into 
relevant mental structures mediating change in sustainability behaviors and food lifestyles. The guiding questions were: (1) which mental models and intrinsic motivations lead to permanent shifts in dietary lifestyles? (2) What are the effects of traits spirituality and mindfulness on food choice and dietary behavior? And (3) how can mental development techniques such as mindfulness, be utilized more efficiently in sustainable consumer sciences? The proceeding subchapters provide answers to these questions by drawing general implications from the presented essays. Furthermore, study limitations and suggestions for further research encounters are provided.

\subsubsection{Tying up loose Ends: Thoughts and Implications}

A common mental theme, found in essays one and two, is personal development. In essay one self-reflective behavior in regard to dietary decision making was triggered through the confrontation with an impactful intrinsic or extrinsic stimulus. On the one hand, these stimuli challenged the formally held mental models guiding dietary decisions. On the other hand, new mental models based on greater self-awareness, self-recognition, and integrity were established to inform future dietary behavior. Similar implications on the importance of self-development were present in the factor construct "affirmative mindset" (MIND I) presented in essay two. Its constituents of self-awareness, self-reflection, and compassion revealed to be a relatively strong predictor of pro-environmental behavior including food choice, transportation and resource use. Furthermore, an affirmative mindset increased dietary awareness thereby supporting a more balanced nutrition and a better understanding of the underlying physical and emotional needs motivating food choice and dietary habits. Based on the results of essay one and two, it seems as if greater levels of self-development in terms of reflexivity and awareness lead to more balanced dietary decisions. These results align with implications given by FRANK and colleagues (2019) who emphasize the importance of self-development parameters to stimulate sustainable consumption. Exploring the parameter of self-reflection, they find that greater levels of introspection motivate sustainable consumerism. This increase in sustainability stems from an increased ability to actively connect to acquired knowledge and personal virtue before consumption 
decisions are made. Hence, self-development characteristics are driving forces shaping mental models as they are directly involved in information processing.

Therefore, a better integration of issues of self-development into public and political debates may therefore prove valuable for long-lasting sustainable development. However, currently employed public strategies to guide sustainable food consumerism mainly focus on, or at least prioritize, restrictive, regulative, or supportive frames (SPILLER et al. 2017) but largely neglect concepts of experiential self-development (He and Greenberg 2008). Through this, sustainable development is largely promoted by extrinsic motivations only. This may prove insufficient in cultivating long-term behavioral change as externally motivated behavior tends to terminate as soon as the frameworks basing them dissolve or prove faulty (DEYOUNG 1993). Further research, therefore, should focus on the deeper understanding of the relationship between personal development and food consumption behavior change. This comprises not only a thorough exploration of the mental models involved with food choice and dietary lifestyle but also how these can be promoted within the broader society.

A more comprehensive understanding of the factors shaping mental models is necessary, too. The concept of mindfulness offers a rich knowledgebase on mental development techniques which hold the potential to reorganize subjective perceptions about the world, others, and the self (ROSENBERG 2004, KUAN 2008). Despite these promising implications, studies relating mindfulness to dietary change behavior (e.g. TAPPER et al. 2009, KEARNEY et al. 2012, JORDAN et al. 2014) or sustainable consumption (e.g. AMEL et al. 2009, ARMSTRONG 2012, GeIGER et al. 2019, Werner et al. 2020) show mixed results. The inconsistencies make it difficult to estimate the true potential of mindfulness techniques on behavior change. A thorough integration of mindfulness into sustainable consumer sciences may, therefore, prove helpful in order to understand how it can be efficiently utilized, cultivated, and assessed. Essay three suggested approaching the relationship between mindfulness and sustainable consumption behavior through frameworks that include aspects of insight, ethics, and morality. These extensions to the currently utilized 
mindfulness framework were drawn from traditional literature on Buddhist mindfulness. Similar suggestions of reintroducing ethics and morality into mindfulness were proposed by GRABOVAC and colleagues (2011), PURSER AND Milillo (2015), and Monteiro and colleagues (2015). Grabovac and colleagues (2011) argue that ethical frameworks decrease doubt, worry, and guilt which lead to a reduction of mental proliferation. Mindfulness then can be understood as a tool for aligning personal ethics and behavior. PURSER AND MILILLO (2015) point out, that the contemporary mindfulness framework is incomplete and can even prove counterproductive, if ethics and morality are not considered, as they reduce the ability to critically engage, discern, and reflect on situations and circumstances. In their article on traditional and contemporary mindfulness, MONTEIRO and colleagues (2015) raise similar concerns in regard to the limited scope of contemporary mindfulness concepts. They see major shortcomings especially in regard to the sustainability of treatment outcomes claiming that, without ethical guidelines only symptomatic relief from distress is provided. Both articles, PuRSER AND Millillo (2015) and Monteiro and colleagues (2015), emphasize the utilization of right mindfulness in Western mindfulness sciences. Accordingly, essay three also builds on the concept of right mindfulness, as it provides useful implications on ethics, morality, and insight. Furthermore, the four essential functions of mindfulness (simple, protective, and introspective awareness and deliberately forming conceptions) are introduced as reference points for scale development. Therefore, the third essay may provide a good starting point for the design of integrated mindfulness concepts into sustainable consumer sciences. Further research encounters should aim at developing scales that capture the aforementioned aspects of mindfulness reliably.

Aspects of ethics and morality were also of importance in the first and second essay. In essay one, animal and environmental ethics revealed to be of importance for food consumption decisions. Ethics including social interactions were considered but of comparably lesser relevance for food consumption decisions. Essay two implied similar tendencies but factor items pointed to an ethical component related to the responsibility towards others. Even though items on animal ethics were not captured in essay two, in sum, these results 
seem to reinforce the close connection between ethics and sustainable food consumerism. Ethically informed political and economic frameworks, like political consumerism or ecological citizenship, may therefore be of crucial importance to the establishment of long term sustainability as consumption decisions are made based on ethical aspects like pro-environmentalism and social justice. This should be further addressed.

In conclusion, this dissertation contributes significantly to a better understanding of the mental processes concerned with sustainable food consumerism. The mayor takeaways are:

(1) Self-reflective mental models encouraging responsibility and authenticity hold the potential to cultivate long-lasting fundamental changes in food consumption behavior. Interestingly, external factors proofed to be important catalysts of such characteristics. For example, key moments in experience often triggered self-reflective behaviors and increased the personal interest in diet and dietary lifestyles. Social parameters like tolerance and acceptance can strengthen dietary confidence and provide reassurance.

(2) Traits spirituality and mindfulness were found to be reliable, conceptually independent, predictors for specific sustainability relevant food choices (pro-environmental behavior and dietary awareness) and dietary (convenience and fruits and vegetables) behaviors. However, results proofed suspicious in regard to other parameters (e.g. animal-protein based foods) reinforcing the significance of properly established measurement frameworks for these traits.

(3) Thoroughly integrated theoretical approaches for exploring mental mechanisms in sustainable consumerism are, therefore, necessary in order to establish a concise understanding of the application, scope, and relevance of abstract mental concepts for behavioral change. For example, commonly applied frameworks for scaling mindfulness usually neglect aspects of insight, ethics, and morality which are traditionally associated with the construct. 
Through this, the presented findings are useful not only to gain insight into the mental models and mind development techniques mediating behavior change, but imply that these traits may be reliable predictors for estimating changes in food consumption behavior and, through that, sustainable development.

\subsubsection{Limitation of the presented Research}

The first essay builds on a qualitative approach to understand motivational drivers and mental models of alternative dietary lifestyles. Qualitative data were collected through face-to-face interviews which required both interviewee and interviewer to meet in the same space. Through this, recruitment methods were limited to a comparably narrow geographical scope, comprising three federal states only. Furthermore, participants were predominately German females over age thirty. This implies that the results are limited in generalizability. However, it should be noted that the study carried out in essay one did not aim at generalizability but on the cultivation of a first understanding of the diverse values, motivations, and inspirations guiding alternative dietary decisions. Future studies are advised to cover additional population subgroups if similar topics are investigated. Through this, a more complete picture of the motivational and mental dynamics informing alternative food choice and dietary behavior is created. Knowledge on the strength of relationship between the identified mental models and diet could be gained through structural equation modeling or other suitable quantitative means.

The data utilized in Essay two were collected online through a research panel provider. While these channels for data collection offer various advantages, like cost efficiency and time saving, they also pose limitations on the results. For example, sampling issues in regard to participation bias were encountered for both country survey sets. In India, low education and income classes could not be sufficiently covered leading to an orientation towards highly educated and comparably wealthy respondents. Within the US sample, somewhat similar issues with education levels were faced. However, in opposition to the Indian sample, the oversampling favored participants with comparably lower education. These issues limit the generalization of the presented findings to a certain degree as some segments of society, besides those who are illiterate or 
don't have access to an internet device, remain unconsidered. Participation bias is a common phenomenon in quote sampling (HEIERVANG AND GoOdMAN 2011). One possible approach for stabilizing the datasets could have been the random elimination of observations from overrepresented subpopulations until representativeness is reached. However, this approach was rejected since it would have led to a major reduction in sample size of the Indian data set where highly educated respondents accounted for $81.3 \%$ instead of $6.7 \%$ of the observations. Through this, study two may be limited in general representativeness but provides valuable information about otherwise underrepresented subpopulations.

The studies presented in essay one and two examined behavior based on selfreport measures (essay 1: interview; essay 2: online questionnaire). For the exploration of this relatively novel study topic these measures were suitable for gaining first understandings on the mental mechanisms and models informing behavioral tendencies. However, in order to verify indicated food consumption behavior with true dietary intake, the inclusion of behavioral measures is required, as self-reported measures are prone to be overstated (KORMOS AND GIFFORD 2014). Suitable behavioral measures for dietary behavior are, amongst others, food frequency questionnaires, food records, and 24-hour recalls (JOHNSON 2002).

Lastly, the concept of spirituality and its justification for scientific exploration is controversially discussed amongst scholars, as it challenges core scientific values (HAYEs 1984, Powell 2001, BouRne 2009). Nevertheless essay two clearly indicates that a greater integration of spiritual values into sustainability sciences can prove insightful for resolving current challenges in consumerism. Trait spirituality, especially the aspect of spirituality that is involved with nature and society (SPIRIT II), showed to have a relatively large positive effect on the consumption of fruits and vegetables and reduced the intake of convenience foods. Furthermore, SPIRIT II significantly reduced the consumption of animalprotein based foods within the Indian subsample whereas a plant-protein based diet was significantly promoted amongst respondents from the USA. Similar mental structures in regard to the relationship with nature and society were also 
found when analyzing the qualitative interviews conducted in essay one. Here, the relationship to the environment and others was based on respect, care, and responsibility. Moreover, for many respondents the connection to nature was experience based rather than intellectual. Such mental models can be considered a part of the spiritual worldview (HEINTZMAN 2009) even though, people not necessarily identify as being spiritual or, perhaps, don't want to be identified as spiritual. However, this rejection of spiritual values may prove faulty as it fails to draw a complete picture on the totality of factors informing decision making and behavior. Furthermore, this strict demarcation between science and spirituality may even prevent or confuse scientific insight PURSER AND MILLILLO (2015). As presented in essay three, in the case of mindfulness, spiritual values were intentionally omitted in order to reach greater acceptance. However, through that important ethical and moral implications remained, and largely continue to remain, unconsidered within Western concepts of mindfulness. Essay three, therefore, contributes to thorough understanding of the concept of mindfulness, including spiritual values. Through that, relevant implications about its potential magnitude of effect as catalyst for behavior change were demonstrated. BOURNE (2009) already pointed to the trend in worldview that connects spirituality with sciences. This openness towards the exploration of spiritual values becomes more and more prevalent also in sustainability sciences. However, research still needs to build a common fundament for the applicable and meaningful integration of both concepts that seem to be fundamentally different in how they approach phenomena. Perhaps, one pillar may be the mutual desire to know more about the world. 


\title{
Chapter 2 What it takes to change: Adoption and Maintenance of Alternative dietary Lifestyles
}

\author{
With DR. ANTJE RISIUS
}

This article has been submitted to Appetite in November 2020

\begin{abstract}
For Western culture, changes in food lifestyles of consumers, in particular reducing the share of animal-based foods within the diet, are of great importance for sustainable lifestyles. However, changes in diet are generally considered difficult to make as eating behavior is strongly interlinked with habit and identity. This study aims at investigating the motivations and mindsets involved with adopting alternative dietary lifestyles to better understand the driving forces guiding change in eating patterns. Qualitative methods were used to collect data on the food-related motivations and mental dynamics of 17 longterm 'alternative dieters'. Analysis of the material revealed three factors as being particularly relevant for changing usually followed eating patterns into new ones. These were the experience of (1) a key moment, the accumulation of (2) knowledge, and (3) health concerns. While the first factor, key moments, catalyzed an immediate behavioral response, change processes of the second and third factors appeared to be more organized. Interestingly, the aspect of information acquisition was important in each process of change. With regard to the mindsets that reinforced these new eating patterns into long-lasting alternative diets, three further characteristics were found. These were the selfreflective (1), responsible (2), or interconnected (3) mindset. Overall, the results provide a first glance at the diverse intrinsic dynamics involved with alternative dieting. Through this lens, a different perspective on change behavior in food consumerism is presented that needs further investigation in order to grasp its potential to stimulate sustainable transformations in food consumption behavior.
\end{abstract}

\subsection{Introduction}

To ensure the well-being of future generations, fulfilling the UN's sustainable development goals (SDGs) remains high on national and international policy agendas. Nutrition is crosscutting through all most of the SDGs either directly or 
indirectly. For the Western world, food lifestyle changes emphasizing plantbased diets have received considerable attention, since the production of meat for human consumption represents a major burden on the environment (Pelletier And Tyedmers 2010, Hedenus et al. 2014). Within the last two years the number of German citizens classifying themselves as vegetarian has increased by 400,000, reaching around 6.1 million in 2019 (PAWLIK 2020a). Germans self-classifying as vegan increased by $13.1 \%$ from 2017 to 2019 (PAWLIK 2020b). Scientific interest in dietary behavior change has been growing over recent decades, reinforcing the crucial role that diets play for sustainable development and human well-being (ALEKSANDROWICZ et al. 2016, WiLLETT et al. 2019). However, a long-lasting shift in food lifestyle calls for behavioral change on many levels, routines, and practices since food demand and supply are closely interwoven.

Within food choice decisions, consumers are influenced by a host of external and internal factors, revealing that food consumption behavior itself results not only from individual preferences and biological predetermination, but also from micro- and macroeconomic circumstances as well as sociocultural influences which make this behavior relatively hard to change (VERMEIR AND VERBEKE 2006, Story et al. 2008). However, research has revealed that changes in behavioral patterns are possible since food choices and diet are also strongly influenced by motivation (VERMEIR AND VERBEKE 2006). Therefore, understanding the motivational structures that lead to changes in dietary behavior is important for successfully implementing sustainable processes of transformation within the food system. Even though food consumption-related motivations have been studied by various researchers (for example, RENNER et al. 2012, PhAn AND Chambers 2016) they have hitherto been limited in their applicability for exploring dietary change behavior. Since food consumption behavior is strongly driven by routine (SPAARGAREN et al. 2013), and is therefore especially hard to overcome, exploring the motivational structures that underpin successful behavior change motivations, may be especially fruitful for understanding how long-lasting changes in dietary behavior, happen. In this regard alternative dietary lifestyles may be of interest in understanding how such changes could be facilitated. Even though they are not necessarily more 
sustainable, alternative dieting is promising in understanding how food paradigms can be transformed as the means to establish and maintain such diets on a daily basis is more difficult as compared to conventional eating behaviors (BATAT et al. 2016). Specifically, it is of interest to look at consumers' motivation forces and how these relate to the establishment of new dietary habits. Further study questions are: What are the main determinants catalyzing change? Are those motivation solely bound to an ideological concept? What other drivers play a role in reinforcing new dietary habits? Which effects do sustainability and general health have?

So far, few studies have been conducted to better understand the intrinsic mechanisms motivating these diets. Furthermore, common theories on changes in health behavior propose behavioral change to be linear and accordingly view it as a step-by-step journey. Nevertheless, non-linear systemic approaches have been put forward to reflect lifestyle changes more accurately (RESNICOW AND VAUGhan 2006, Cerin et al. 2009, Boudreaux et al. 2012).

In this regard, psychological and structural processes that underlie alternative food lifestyles may prove valuable in gaining deeper insights into the issue of dietary behavior change. The present study therefore focuses on the factors that have motivated consumers to change and maintain an alternative food lifestyle. By this approach, the authors attempt to foster a more nuanced understanding of the components involved in making extreme changes in dietary behavior. Knowledge of these factors may provide interesting insights that could be transferred to the sector of sustainable development in diets as it may point to the fundamental mindsets necessary to support transformation processes in dietary behavior. Our specific research questions are therefore: (1) what motivated consumers to adopt alternative styles of nutrition? And (2) what were the mindsets reinforcing these changes over a prolonged period of time? The overall aim is to understand which intrinsic motivational drivers are involved with long-lasting dietary lifestyle changes and how these lifestyle shifts have been undertaken. Based on the outcomes of this study we intend to recommend possibilities for an enhanced understanding of such motivational structures for policy makers, nutritionists and society in general. 


\subsection{Methods}

\subsubsection{Activity-based in-depth Interviews}

Apart from veganism, which has been gaining growing attention within the interdisciplinary research community, very little research has been done on the motivational drivers of alternative food practices and the implication for transitions to wide-ranging sustainable food practices. According to MIHAS (2016), qualitative methods are suitable for addressing research questions if a less explored field of research is entered and literature is scarce. Furthermore, open questioning allows the researcher to gather new knowledge and thereby fosters a better understanding of diverse personal and social interactions, modes of meaning, and behavior compared to the use of more standardized, closed empirical formats (TeddLIE AND TASHAKKORI 2009, TRACY 2019). This study aims to investigate the subjective perceptions and intrinsic psychological mechanisms common to followers of alternative diets. In order to stimulate different psychogenic planes, the interviews were enriched with activities. Each session followed the same structure: introductory conversation - activity 1 discussion about activity 1 - conversation topic - activity 2 - discussion about activity 2 - conversation with final remarks and questions.

Each interview began with an explanation of the research purpose as well as an assurance of data privacy protection. Conversations were based on guiding questions. These were framed around two conceptual themes: (a) the motivational drivers for adapting a particular food lifestyle, and (b) major influences that led the subject to maintain their chosen alternative food lifestyle. However, the order and specific phrasing of the questions varied according to participant and interview flow.

Both activities consisted of the arrangement of pre-written statement stickers on a paper sheet. Participants were encouraged to arrange the statements according to their own preference in the absence of the interviewer. Furthermore, self-written statements as a supplement to the pre-written statements could be added by the participants if necessary. The first activity focused on individual eating motivations. The motivations used were derived from The Eating Motivation Survey (TEMS) conducted by RENNER and 
colleagues (2012). This questionnaire proved to appropriately reflect the fundamental eating motivations common in German society (SPROESSER et al. 2018). The 15 motivations were: (1) liking, (2) habits, (3) need and hunger, (4) health, (5) convenience, (6) pleasure, (7) traditional eating, (8) natural concerns, (9) sociability, (10) price, (11) visual appeal, (12) weight control, (13) affect regulation, (14) social norms, and (15) social image. These motivations were arranged on a blank sheet of paper. The second activity focused on the exploration of identity and worldview characteristics in order to gain deeper insight into the mindsets maintaining long-term dietary changes in the participants. For this activity, statements of the Vital Impact Assessment Scale (VIVO) conceptualized by PÉREZ-SALES and colleagues (2012) were used. This scale was selected as it accounts for a diverse range of experiential response mechanisms and fundamental beliefs shaping mentality. In order to randomize the 30 statements from the original 116-item VIVO questionnaire, a random value formula provided by the program Excel 2010 was applied. Nevertheless, all interviews covered the following ten conceptual areas: (1) worldviews, (2) attitude towards the world, (3) view of human beings, (4) coping, (5) impact of past situations, (6) emotions, (7) telling the experience, (8) consequences, (9) social support, and (10) identity. Participants were given a sheet of paper with an arrow drawn on it (see figure 2.1). The 30 statements were arranged in a Likert-scale manner based on their correctness for the participants' characters.

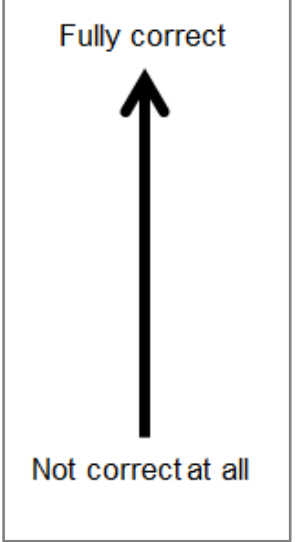

Figure 2.1: The sheet provided for activity 2. The 30 statement stickers, derived from the VIVO questionnaire, were arranged in a Lickert-scale manner based on the correctness of the statement for the participants' characters. 
For both activities, explanatory sheets were available in order to resolve any confusion with regard to the meaning of the motivations or statements. The arrangement of all motivations and statements was advised but not mandatory. An example of the activities is given in appendices A.2 and A.3.

After the activities had been accomplished, they were discussed in the following part of the interview to further understand the arrangement of statements as well as the personal interpretation and meaning of the statements. If participants chose to dismiss some of the given statements, these were neither discussed nor used in further analysis.

After the in-depth interview a short questionnaire, capturing socio-demographic data and specific dietary habits (purchasing behavior and components of diet), was filled out by each participant. Furthermore, after each interview, a short protocol of the environment in which the interview took place as well as a subjective assessment of the situation (for instance, mood of interviewee and interviewer, personality characteristics, and body language) was obtained.

The interview duration was estimated for a scheduled time of 90-100 minutes. However, the average time for an interview was 119 minutes, with the shortest interview taking 92 minutes and the longest interview lasting 134 minutes. Data collection took place between July and October 2019 in the Federal States of Lower Saxony, Hessen, Baden-Württemberg, and Bavaria. Interviews were conducted in German. Participants were rewarded with a financial compensation of $€ 20$ per person (\$22.19 as of 20.12.2019). All interviews were recorded and professionally transcribed before further data analysis.

\subsubsection{Participants}

The target group comprised people who had consistently followed an alternative dietary lifestyle for at least one year. Since there is no commonly agreed upon definition for the term, we use the concept provided by the NATIONAL RESEARCH COUNCIL (US) in 1982. An alternative diet is defined as "any diet used by an identifiable group of people larger than a family". This definition was further extended by aspects provided by BATAT and colleagues (2016) comprising the 
presence of shared values and common ideologies like food literacy, environmental concerns, or food social justice.

Participants for this study were recruited through three different means, namely (1) announcements on social media platforms and in public spaces like health food stores or universities, (2) flyers in the offices of nutritionists, alternative practitioners, and left-leaning political groups, and (3) 'snowball sampling' and word of mouth. All participants were introduced to the ethical procedures of the data collection and gave their written and oral consent.

\subsubsection{Qualitative Content Analysis}

The qualitative content analysis of the transcribed interviews followed a system proposed by PERCY and colleagues (2015). The 11 steps applied were: (1) defining relevant material per participant, (2) identification of relevant information, (3) review of data and exclusion of irrelevant information, (4) coding of data, (5) clustering of data/identification of patterns, (6) further identification of data that fit the pattern, (7) combining related patterns into themes, (8) arrangement of themes in a matrix, (9) detailed description of themes, (10) identification of themes common to all participants' data, and (11) synthesis of data to answer the research question.

Analysis was conducted using the statistical software program MAX QDA.

\subsubsection{Analysis of activity based Material}

After each activity, the collected sheets were discussed with the participants to ensure the proper interpretation of the material gathered. The 15 motivations collected in activity 1 were ranked according to importance. However, each rank could be shared by various motivations. If the ranking was not clearly indicated on the sheet, the corresponding interview material was evaluated for further clarification. Activity 2 was analyzed by extracting the three to five most prominent characteristics on each end of the arrow. Where necessary, the corresponding interview material was used to further specify the derived characteristics. 
Each analysis was performed independently by two people. Results were matched, clarified, and merged in a follow up process. Microsoft's Excel was used to analyze the results.

\subsubsection{Sample Description}

Following grounded theory to qualitative research, data collection was carried out until data saturation was reached (BODDY 2016). The material was constantly reviewed and matched. After completing eight interviews preliminary content analysis was conducted. The results pointed to the necessity to collect further material in order to capture the complexity of the issue under observation. Therefore, further interviews were conducted and simultaneously analyzed. After 17 interviews the material revealed to have reached the point of data saturation. The demographic profiles of the participants are depicted in table 2.1.

Table 2.1: The demographic profile of the 17 interviewees participating in the alternative dietary lifestyle motivation study. Data were collected through activity-based, in-depth interviews in Germany from July to October 2019.

\begin{tabular}{llll}
\hline \multicolumn{3}{c}{ Demographic profile of the interviewees $(\boldsymbol{n}=\mathbf{1 7})$} \\
\hline \multicolumn{1}{c}{ Age } & \multicolumn{1}{c}{ Income } \\
$<26$ years & 2 & $<1000 €$ & 2 \\
26 - 35 years & 9 & $1000-2000 €$ & 4 \\
36 - 55 years & 4 & $2001-3500 €$ & 5 \\
$>55$ years & 2 & $3501-5000 €$ & 2 \\
& & $>5000 €$ & 1 \\
& & N/A & 3 \\
\multicolumn{1}{c}{ Gender } & & Education & \\
diverse & 1 & Upper secondary & 4 \\
female & 12 & A-level & 13 \\
male & 4 & & \\
\hline
\end{tabular}

Of the 17 people interviewed, four were male and one diverse gendered. The remaining 12 participants were female. All but one participant were of German nationality. The average age of respondents was 36.6 years, with three participants younger than 30 and three participants older than 50. All 
participants had received higher education, either upper secondary education (Fachhochschulreife; $n=4$ ) or an advanced level (Abitur; $n=13$ ). With regard to their monthly income, six participants earned up to $€ 2000$ or less ( $€ 1000$ equals $\$ 1108$ as of 20.12.2019). Five participants earned between $€ 2001$ and $€ 3500$ per month and three participants had a monthly income of more than $€ 3500$. The remaining three participants where either unsure about their monthly income or chose not to respond. Many participants worked in areas related to health or the environment. Seven participants were either nutritionists or worked in fields closely related to nutrition (for instance as a health food chef). One reason for this may lie in the mode of sampling. Amongst others, flyers for display were distributed to offices of nutritionists and appeared to catch the interest of the practitioners as well.

In total, eight alternative dietary lifestyles were captured, namely: (1) Vegan diet $(n=5)$, (2) Five Elements diet derived from Traditional Chinese Medicine (TCM; $n=3)$, (3) Freegan diet $(n=2)$, (4) Macrobiotic diet $(n=2)$, (5) Intuitive diet $(n=$ 2), (6) Anthroposophic diet $(n=1),(7)$ Yogic diet $(n=1)$, and (8) Chrono diet ( $n$ $=1$ ). Table 2.2 provides an overview of the main characteristics of each alternative diet sampled. 
Eastern philosophies. However, participants following the vegan and freegan food lifestyle $(n=7)$ reported they were atheistic or agnostic.

The mean average of how long participants had been following the corresponding alternative food lifestyle was 7.6 years; the median value was five years. The longest duration for following an alternative diet was 22 years and the shortest two years.

\subsection{Results}

\subsubsection{General Motivations for dietary Lifestyle Changes}

Almost all participants had followed a vegetarian diet before transitioning to their current dietary lifestyle $(n=14)$. For most of these participants the change from a meat-inclusive diet to a vegetarian one was linked to ethical issues concerning the consumption of meat. These issues included the suffering involved in producing animal foodstuffs as well as environmental concerns. Of the 14 interviewees, two grew up vegetarian and had never eaten meat products in their lives. When analyzing the textual material, three different recurring phenomena were identified that accompany the transition process from usual (whether vegetarian or meat-inclusive) eating pattern to alternative diet. The three different transitioning processes are depicted in figure 2.2.

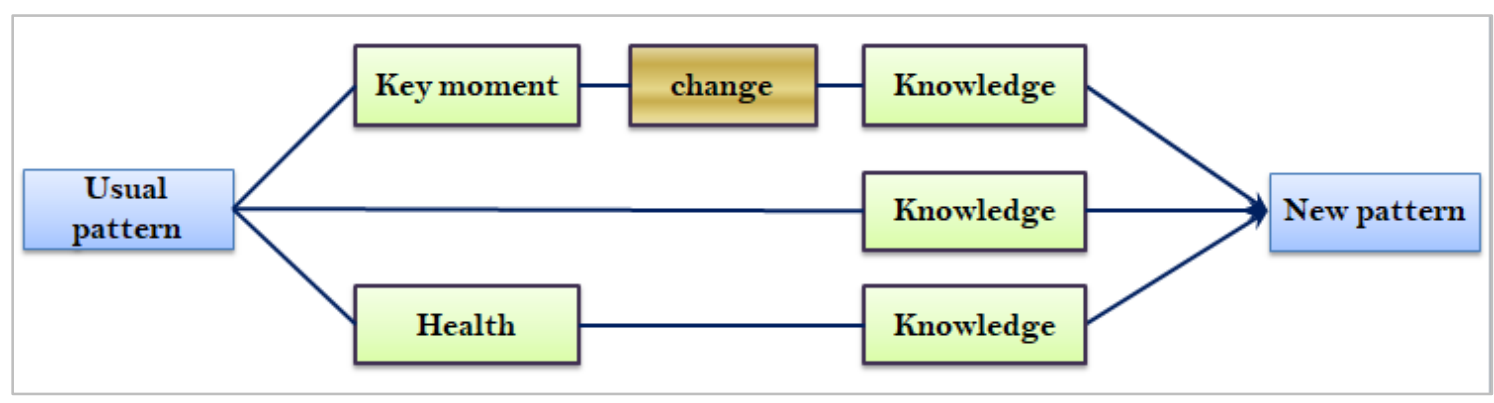

Figure 2.2: The three recurring patterns found when transitioning to a new dietary pattern. Results are based on interview data collected from participants following different alternative dietary lifestyles.

For most participants the experiencing of a key moment (1) appeared to be particularly relevant for food lifestyle transitions. Here, change was induced by a sudden insight to the mind. Key moments comprised experiences that triggered a strongly affective response, like great admiration for or disgust towards a 
person, structural system, or social group. Such key moments caused the participant to implement immediate and specific change - for example, the immediate exclusion of a certain food product or type of processing from their diet. In most cases additional information on nutrition in general or on a specific alternative diet in particular was sourced before participants transitioned to their new dietary pattern. In contrast to the first, experience-based route, the second process was mostly built on knowledge (2). In this case, participants changed dietary lifestyles after they had accumulated enough evidence to logically support their transition towards an alternative lifestyle. Often information was gathered and absorbed passively at first before it turned into an active collection of additional knowledge about a specific dietary lifestyle. Changes in eating patterns were mostly timed based on a specific transition period or on the final consumption of already stocked food items that did not fit participants' new dietary style. The third type of change related to health concerns (3). These concerns comprised personal diagnosis (discovering one has diabetes, for instance) as well as health issues within their close family circle. These concerns motivated participants to reconsider their personal dietary lifestyles and source knowledge regarding alternatives types of diet before changing their previously followed eating patterns.

\subsubsection{Mentalities of pertinent Dietary Changes}

Analysis of activities 1 and 2 enabled identification of those mindsets that contributed towards transforming newly established dietary patterns into longlasting alternative food lifestyles. Three commonly shared themes were noted. These were (1) self-reflexivity, (2) responsibility, and (3) interconnectedness.

\subsubsection{The self-reflexive Mindset}

Compared to their former eating behaviors, interviewees described a considerable increase in well-being and vitality, both physically and mentally, taking place soon after they had changed their food lifestyle. When asked for more details, they referred to an increase in energy levels and an ability to focus as well as the absence of strong appetite or feelings of heaviness. Furthermore, many participants reported that conventional Western food habits and/or combinations, for example the consumption of highly processed foodstuffs, 
animal products, or ready to eat foods, no longer satisfied them, did not taste good, or even led to feelings of malaise.

"For the quality of life alone [..]. It gives me the freedom to feel the way I want to feel. [...] It was always my desire to feel comfortable, happy, vital, and balanced. [...] And then I noticed that I've become more balanced and stronger on all levels of health, simply much more stable, and I reached that through changing my diet." (A08)

Alongside this state of elevated physical and mental well-being, it seems as if the continuous setting and defending of dietary boundaries was perceived as encouraging and supporting participants' feelings of self-worth. As a result, interviewees felt more authentic and a stronger sense of connection to the self. Participants often mentioned that the necessity of communicating the changes in their lifestyles to family, friends, and wider circles of contacts made them more courageous, not just with regard to their food lifestyles but also with respect to other areas of their lives.

"I used to feel obligated to do or eat certain things but I became more mature somehow.

[...] I know it's my decision. I don't have to do anybody a favor." (A09)

Many participants reported that they had started to express the behavior they would like to receive themselves, such as showing respectfulness and appreciation for individual wishes and having fewer expectations concerning how others should be or what they should eat. Others reported a greater sense of self-reflection which motivated them not only to spend a great deal of their time reflecting upon personal ethics, beliefs, and values, but also to find solutions to perceived difficulties.

\footnotetext{
"At the beginning you'll have trouble finding the right food items. You'll spend a lot of time in the supermarket [...]. I've walked this same path before. I know how difficult it is. If I see you, then I feel it's on me to help you. If I see you and I realize you need help, it's my responsibility to reach out to you. It's not a matter of a question for me, it's something I have to do." (A15)

When I realize that I'm stuck and my circumstances can't be changed, then I sit down and I focus on the parts within this circumstance that I can change." (A17)
} 


\subsubsection{The responsible mindset}

The majority of participants exhibited a strong sense of responsibility that they had developed towards others or the environment. In some cases, this heightened sense of responsibility led the participant to feel as if the burdens of the world lay only on their shoulders. This was especially true for vegan and freegan interviewees.

In terms of social responsibility, there was a clear idea of where responsibility begins and what this should require from society as a whole. Many participants perceived themselves as role models, sources of motivation for others, or rebels in bringing forward a new food-conscious society based on alternative values. This self-created image was highly associated with the motivation to maintain their respective food lifestyle no matter how difficult the circumstances were, so as to not concede defeat to themselves or others.

"Also I'm a role model for the children, for the sick children, when I work with them so that they see that I'm a healthy person myself. [...] I can treat the children that way and I embody what I speak of. (A07)

Generally, respondents were knowledgeable in sustainability and environmentally related challenges and complexities. In many cases, they had clear opinions on the societal and structural steps necessary for resolving environmental problems. Yet, in response to this knowledge, some interviewees had developed a strong sense of personal responsibility towards nature and the environment which reinforced their choice of alternative dietary lifestyle. Here, nature was perceived as being in urgent need of preservation and care.

"People in themselves are the problem and, accordingly, [...] it's one thing to say that, as a human being, you try to reduce your own consumption as much as possible but, viewed from another perspective, every person living frugally still has a larger $\mathrm{CO}_{2}$ footprint than every person who does not live that way." (A05)

\subsubsection{The interconnected Mindset}

In contrast to the responsible mindset where the motivation to maintain the alternative diet stems out of an idea of accountability, the interconnected 
mindset was more affectively biased towards an idea of unity with all living things.

With respect to nature and the environment, experience-driven emotional connections were mainly described by participants who were somehow connected to a farm (for instance, their grandparents lived on one), who themselves grew up on a farm or were raised in rural areas, at least for parts of their childhood. Nature was described as being nurturing, calming, or harmonizing in general. Participants expressing this kind of mindset predominantly followed dietary lifestyles that emphasized harmonizing and aligning the body with spiritual or natural forces (for example, TCM, Yogic diet, and Chrono diet).

"And we had a farm, in Poland, and every time we went there I felt very happy, like in these movies where the kids play with the goats and feed the chickens and run through the open fields. [...] During this part of my childhood I felt very content, more myself somehow." (A15)

The role of society as a whole and personal social networks in particular appear to be important driving forces for specific changes to food lifestyle. While TEMS motivations provided by "social norms" and "social image" were of low importance or even strongly rejected, the aspects of social support and social inclusion were considered important, motivating, and desirable. Approximately half the participants referred to social support from friends, family members, and specific social groups, as a major inspiration for continuing their alternative food lifestyle. Furthermore, while most participants felt supported by their closest contact networks (family and close friends), almost all interviewees mentioned the wish for greater tolerance, respect, openness, and acceptance from society as a whole, and especially at work.

"Especially from those who knew me, I expected more tolerance. [...] From the society I cannot ask this, of course it would be great, but strangers mostly don't want to understand." (A11) 


\subsection{Discussion and Conclusion}

This empirical qualitative study has aimed to explore the motivational drivers and mindsets inducing and reinforcing changes in dietary patterns. As far as the authors are aware, this paper is one of the first to investigate this phenomenon by using a sample of people who have followed diverse alternative food lifestyles over a prolonged period. The findings imply the existence of common characteristics involved in adopting and maintaining alternative diets. Furthermore, viewed through a wider lens, they point to existing structural and social barriers that may hinder consumers from abandoning their usual patterns of dietary consumption.

With regard to the adoption of alternative food lifestyles, this paper found three factors to be particularly important. These were (1) the experience of key moments, (2) the accumulation of knowledge, and (3) health concerns. Changes in dietary behavior were most often instigated by eye-opening moments and insights which greatly affected the way participants perceived their personal diet or the system of food production as a whole. These results align with findings on non-linear behavior change made by RESNICOW AND VAUGHAN (2006). However, it should be noted that while there was an initial shift within participants' dietary habits, this was often accompanied by a period of modification where further knowledge was acquired before people settled into a new, alternative, dietary pattern. It is interesting to note that gathering knowledge represented an essential driver in all three transition processes presented in this article, however, different pathways of motivation were driving this. Nevertheless, factor of knowledge hence seems to be a key factor in guiding people onto different dietary pathways.

This finding could be especially relevant for pathways for enhanced sustainability within the society. In a study of more than 4,000 consumers from four European countries, GRUNERT and colleagues (2014) explored the general understanding of sustainability information for different foods. Even though most participants had a general idea of the meaning of sustainability, there was widespread confusion surrounding the overwhelming number and variety of sustainability labels on food items; similar findings have been reported by 
HANSS AND BÖHM (2012). Both studies point to the importance of proper contextual understanding of sustainability before purchase behavior shifts towards more sustainable choices. These implications align with the findings reported here. All respondents invested time in acquiring proper information before adopting new dietary patterns. However, all participants were also highly educated. The ability to acquire, analyze, retrieve, and organize information may therefore be more pronounced when compared to people with lower educational levels. Hence, alongside the need to provide a proper information framework to consumers seeking to change their diet, it may be equally crucial to design material (for example, fair trade sourcing, environmentally friendly packaging and advertising) that is comprehensible, precise, and easy to access. Furthermore, through the purposeful cueing of information about sustainable food consumption, consumers may be stimulated to rethink their usual dietary patterns (CORNELISSEN et al. 2008).

Three different mindsets (self-reflective, responsible, and interconnected) have been revealed to be important for bridging new dietary habits into long-lasting dietary lifestyles. The majority of interviewees showed evidence of having established a self-reflective mindset. Based on this outcome, the authors propose that if people are more in tune with themselves they appear to be more capable of making independent decisions concerning their diet. However, the issues of self-awareness, authenticity, and integrity are rarely discussed in the current literature around food lifestyles. When they are discussed, the focus is often on a self-imposed personality that the dieters want to align with. Hence, if people do not stick to their particular food lifestyles they feel less authentic (see, for example, CHERRY 2015 and ROSENFELD 2019). While various studies have reinforced this view, this paper implies that certain levels of self-recognition and awareness may be necessary prerequisites to recognize, evaluate, and defend personal value structures and beliefs surrounding dietary decisions. These results align with findings identified by FRANK and colleagues (2019), who emphasize the importance of self-development parameters to encourage patterns of sustainable consumption. In their study on the influence of mindfulness training on sustainable consumption behavior they found that greater levels of introspective awareness motivate sustainable consumption. 
This increase in sustainability stems from a heightened ability to make active connections to acquired knowledge and personal virtue before consumption decisions are made. Therefore, a better integration of issues of selfdevelopment into public and political debates may therefore prove valuable for long-lasting sustainable development in food systems. Current public strategies for guiding sustainable food consumption mainly focus on (or at least prioritize) restrictive, regulatory, or supportive frameworks (Spiller et al. 2017) but largely neglect concepts of experiential self-development (He and Greenberg 2008). In this conception, sustainable development is largely promoted by extrinsic motivations only. This may prove insufficient for cultivating long-term behavioral change as externally motivated behaviors tend to stop as soon as the frameworks supporting them dissolve or prove inadequate (DEYOUNG 1993). Further research should therefore focus on more deeply understanding the relationship between parameters that govern personal development and changes in food consumption behavior. This would not only entail a thorough exploration of the mindsets involved in food choice and dietary lifestyle but would also include suggestions for how these mindsets can be more broadly fostered within society.

With respect to the 'responsible' and 'interconnected mindset', an interesting distinction between followers of alternative diets was noted. The responsible mindset was mostly evident in participants following vegan or freegan dietary lifestyles. Dietary lifestyles like TCM, Yogic diet, Chrono diet, Intuitive diet, and Anthroposophic diet were more inclined to exhibit interconnected mindsets. A reason for this may lie in ideological differences between the respective dietary lifestyles. While diets connected to the responsible mind place a focus on mitigating environmental, social, and ethical issues around food consumption problems (Pietrzyk 2006), diets of participants expressing interconnected mindsets appear to be more inwardly focused at balancing the individual's body and mind (see also LeITZMANN et al. 2005). Strong connections between ideology and food choice have been reported by various researchers (POLLARD et al. 2002, GIFFORD AND CHEN 2017). As a psycho-social factor, ideas about food are comparably easier to alter than socio-demographic aspects and this makes the various ideas important catalysts for changing food consumption 
behavior (CONNER AND NORMAN 2005). Therefore, they are also important promoters of transition processes in diets when it comes to sustainability.

However, aligning behavior towards greater sustainability cannot be built on mental resources alone but needs to be supported through structural changes as well. Almost all participants ranked the motivations "social norm" and "social image" low, if not the lowest, and stated a desire to disassociate with the common social conduct around food and diet. Yet, aspects of social appreciation and support revealed themselves to be important inspirations for adopting and maintaining a chosen dietary lifestyle as manifested through the interconnected mindset. Similar findings on the importance of social networks for diet and dietary change behavior were identified by various researchers (Cherry 2006, Delormier et al. 2009, Vaterlaus et al. 2015) and are reinforced by the findings from our study. Furthermore, current food supply structures pose great barriers to the adoption and maintenance of alternative diets in everyday life (COLE AND Morgan 2011, Bresnahan et al. 2016). The establishment of large-scale sustainable food structures (involving spatial and geographical planning or law making, for instance) is therefore a necessary prerequisite for supporting consumers in transitioning towards greater sustainability. However, such structural changes must include frameworks that promote sustainable values in relation to mutual understanding and personality development in order not just to reduce barriers but to promote behavioral change.

In using a comprehensive qualitative study framework, our results have been based on a selective number of participants $(n=17)$. Even though the number of participants seems relatively small, it was based on a grounded theory qualitative research framework. Within this framework data are collected until saturation is reached regardless of the sample size (BODDY 2016). Amongst the participants, seven were engaging in nutrition focused professions. Therefore, the question of analytical bias may be of concern as these professions are likely to have a better understanding of nutrition (MCCONNON et al. 2004). However, the main research interest of this study was to disentangle the facets and drivers for long-lasting dietary behavior change in consumer's systems 
approach taking into account facets of ideology, health, sustainability as well as communication. This circumstance was therefore not considered problematic for the exploration of this relatively novel research area. In fact, these variables were appropriate for adapting existing theories and hypothesis. It suited to elaborate key drivers of long-lasting change in dietary behavior as well as into the mindsets underpinning such change. Nevertheless, the studys' findings should be validated in quantitative follow-up work. 


\title{
Chapter 3 The Yoga of Sustainable Diets: Exploring Consumers Mind and Spirit
}

\author{
With Prof. Dr. Achim Spiller and Dr. Stephan G.H. Meyerding \\ This article has been published in the Journal of Cleaner Production, 2020, \\ volume 243, pp. 118473.
}

\begin{abstract}
Unsustainable food choice and dietary behaviors put pressure on ecosystems and supply chain structures worldwide. Knowledge on the intrinsic values driving food consumption behavior are, therefore, essential in order to foster sustainability on a global, national and community based level. This study aims at exploring the relationship between different food consumption related behaviors and the intrinsic value shaping constructs of spirituality and mindfulness. Online questionnaire data on self-reported dietary behavior, selfreported food choice behavior, spirituality, and mindfulness were drawn from a population subsample in India and the United States of America. Factorial relationships between spirituality and mindfulness traits were explored using exploratory factor analysis (EFA). Furthermore, multiple regression analysis was performed to estimate the effect of the derived factor structures on different food choice and dietary behavior variables. The EFA resulted in 4 factors, of which two each frame spirituality and mindfulness. These factors are: (1) metaphysical spirituality, (2) physical spirituality, (3) affirmative mindset, and (4) scattered mindset. In both country data sets, factors 2 and 3 had significant adverse effects on the consumption of convenience foods and a positive effect on the consumers' dietary awareness. Even though ambiguities exist concerning important dietary sustainability measures, e.g. the consumption of animal-protein based foods; this research reinforces the value of spirituality and spiritual practice measures for sustainability research, especially for consumer sciences. Further elaboration and application of these concepts may prove valuable for various stakeholders involved as they point to a more integral aspect of human psychology and behavior.
\end{abstract}




\subsection{Introduction}

Stakeholders agree that the shift in global dietary patterns towards increased demand of resource intensive food inflicts with the aim of a sustainable transition of the global food systems (MASON AND LANG 2017). As a response, food consumption behavior has received considerable attention within the national and international community (SPAARGAREN et al. 2013, AlSAFFAR 2016, Evans et al. 2017, MeYBECK AND GITZ 2017). Research has been applied in various fields to better understand the diverse factors shaping human diets (e.g. SANNE 2002, DREWNOWSKI 2009). In regard to consumption behavior, modern approaches have shifted away from classical rational choice models by including concepts derived from different scientific disciplines such as sociology, anthropology, psychology, and human geography resulting in more complex, value based approaches (POWER AND MONT 2010). In this context, research on spirituality and spiritual practice, and in particular mindfulness practice, have been increasingly explored concerning their potential to foster sustainability. Though the concept of spirituality contradicts with core scientific principles and is therefore viewed with great skepticism (POWELL 2001, HAYES 1984), research on spirituality and spiritual practices in the scientific fields of psychology, sociology, and medical health care (e.g. ISAAC et al. 2016, MASON et al. 2016, TANG 2017, HAWKINS et al. 2010) has shown its effect on changing human behavior. Yet, the application of spirituality and spiritual practice within the field of sustainable consumer behavior is still relatively new, thus exhibiting limitations concerning applicability, conceptualization and operationalization (FISCHER et al. 2017).

Limitations relating to the applicability mainly comprise the subjects under investigation. Most studies focus on very specific population sub-samples such as students, obese people, or clinical patients (e.g. CHAIRY 2012, MASON et al. 2016, HaRizAN AND RAHMAN 2017). Another limitation is the conceptualization of the spirituality construct. While the conceptualization of mindfulness is clear and widely agreed upon, difficulties regarding its operationalization remain prevalent. 
This study is an expansion on the topic of worldviews and intents to explore consumers' personal spirituality and the practice of mindfulness and how these two reflect on food related behaviors. Measures are based on population samples drawn from two culturally, economically and environmentally different countries, India and the USA. A factor analytical approach is used to extract applicable structures for measuring spirituality and mindfulness. The four extracted factors are: metaphysical spirituality, physical spirituality, affirmative mind and scattered mind. Furthermore, implications on the effects of the established constructs on different sustainability relevant food choice (informed food choice, pro-environmentalism, and dietary awareness) and eating behaviors (convenience, animal-protein based, plant-protein based, fruits and vegetables) of consumers are estimated. Through this, we are able to point to the core aspects of spirituality and mindfulness that are promising in regard to the evaluation of different food choice and dietary behaviors.

Furthermore, this study adds to the body of consumer studies currently emerging around worldviews, especially concerning the issue of spirituality and its meaning for sustainable development within the food domain. Our results provide valuable knowledge on the intrinsic motivational dynamics shaping sustainable food consumption and are therefore relevant for scientists within the field of sustainable consumer research. Moreover, the findings provide implications for stakeholders, showing what dynamics may prove supportive in fostering a long-lasting sustainable dietary change within the broader society. Before introducing our study we first provide a brief background on how spirituality and mindfulness relate to relevant food choice and dietary behavior issues and point to interesting studies already applied in this research field.

\subsection{Literature research}

\subsubsection{Theoretical Considerations}

Nowadays, most scientists agree that individual food consumption behavior, including shopping, diet, and food waste management, cannot be separated from the cultural, psychological, and social environment the individual is exposed to (MINTON et al. 2015). This seems to make a shift towards sustainable practices more difficult since deeply conditioned dynamics, such as 
the cultural background, may be difficult to change. While some food consumption behaviors may be based upon rigid factors like social norms, personal health and economic offset, changes towards more sustainable eating practices are possible since there are also many motivations involved in making food consumption choices (VERMEIR AND VERBEKE 2006). The understanding of these dynamics especially in regard to sustainability related food behaviors has been and remains a growing field of research. Over time, classic economic and marketing theories were refined and translated into other models including determinants of habit, emotion, social context, and moral aspects (e.g. SCHWARTZ 1977, AJZEN 1991, STERN 2000). Consequently, the relationship of more subtle factors and sustainable consumption behavior has started to receive growing attention. Especially, the Norm Activation Model (ScHWARTZ 1977) and the Value-Belief-Norm Theory (VBN) (STERN 2000) offer insightful theoretical frameworks for the understanding of how intrinsic dynamics translate into behavioral outcomes. SCHWARTZ (1977) proposes that behavior originates from two characteristics, namely (1) a personal consciousness and (2) a feeling of responsibility for the consequences that a certain behavior will have for the environment. STERNS VBN Theory (2000) ties in with these implications by suggesting that behavior isstimulated by three core values which are of either (1) altruistic, (2) egoistic, or (3) biospheric origin. Altruistic values are oriented towards societal well-being whereas egoistic values are based on individual well-being. Issues regarding environmental well-being are called biospheric values. According to Stern's implications, individuals are likely to take responsible actions if their values are endangered to be violated.

Originally, both theories were proposed to explain pro-environmental consumption behaviors. Hence, their scope to explain food choice and dietary behavior is limited. For these behaviors the Attitude-Behavior-Context Theory (GUAGNANO et al. 1995) provides a more adequate theoretical base. According to this theory, behavior is a context dependent interactive process based on personal attitudes. That is, behavior is only activated by personal attitudes if the external conditions like financial, social, and legal situation are neutral. 
Within the context of sustainable food choice and dietary behavior this theory may explain why different behaviors are expressed even though it does not align with the personal attitude towards sustainability, e.g. when green products are not purchased due to a high price even though the individual has a positive attitude towards them. A further extension to these theories was developed by ZEPEDA AND DEAL (2009). Their Alphabet theory aims at explaining food purchase behavior. Its explanatory fundament is built upon VBN theory, ABC Theory and four additional factors, namely (1) habit, (2) knowledge, (3) information seeking, and (4) demographic data. According to this theory, attitudes are formed through VBN as well as through knowledge and information seeking. The translation of these attitudes into context dependent behavior is mediated through habit. In fact, habits play a key role for expressing food choice and dietary behavior, especially, when external conditions are stable (Zepeda AND Deal 2009, Sainsbury et al. 2018). The extent to which habit predicts behavior is determined by the degree of mastery and the perceived control over the performed behavior (HALL AND FONG 2007). This implies that, if there are no changes in contextual parameters as well as a sufficient amount of perceived mastery and control over food choice and diet, changes in behavior may be initiated through changes in habitual behavior (SAINSBURY et al. 2018) or motivational drivers (VERMEIER AND VERBEKE 2006).

Both spirituality and mindfulness are promising driving forces in shaping and reshaping intrinsic value structures. Mindfulness is considered important for the deviation of customary practices and habits whereas spirituality affects intrinsic motivational structures. In regard to food consumption behavior both constructs may support scientists, businessmen and politicians in (better) understanding issues of consumer food choice and dietary behavior but also in navigating consumption behavior towards more sustainable practices, e.g. reduced meat consumption, environmentally-friendly produced food products, and food waste management.

\subsubsection{The Role of Spirituality in Food Choice and Diet}

Although initially sneered as strictly esoteric practices, spirituality and mindfulness have become of interest to researchers of various scientific 
disciplines. This development, starting almost 100 years ago, is a result of a fundamental shift in the societal and scientific understanding of psychology (POWELL 2001). Over time, spirituality and mindfulness became important measures for human behavior, especially in the therapeutic, sociological and psychological context. In consumer sciences, the importance of both measures has also been gaining growing attention, with more and more studies focusing on the various benefits and limitations of these two concepts for determining consumer behavior (FISCHER et al. 2017, RODRIGUEZ-RAD AND RAMOS-HIDALGO 2018). This is especially true for the concept of spirituality which, until recently, has been strongly associated with religion but is now perceived as a separate psychological construct (HILL et al. 2000). The distinction between spirituality and religion in the scientific discourse proves important as it helps to understand modern societal developments where more and people increasingly identify as spiritual but not religious (HILL et al. 2000, BALL et al. 2001). Nowadays, it is commonly agreed upon that religion may be part of one's personal spirituality, but spirituality does not necessarily require religious belief.

Despite the conceptual distinction of the term spirituality, its definition and clear delineation remains challenging and various interpretations are used. Screening through the available literature on spirituality, it is striking that there are numerous definitions and perspectives on spirituality. As an example, in 1999, PARGAMENT defined spirituality as the "search for the sacred". Almost 15 years later, DE JAGER MEEZENBROEK and colleagues (2012a) referred to spirituality as "one's striving for and experience with the essence of life". Those two examples highlight the inherently abstract character of the term itself, which poses great challenges to its validation and measurement. To overcome these terminology issues, KALE (2004) conducted an extensive literature research and summarizes spirituality as "the engagement to explore- and deeply and meaningfully connect one's inner self - to the known world and beyond". He further summarizes the term spirituality according to four dimensions: (1) a sense of the inner self, (2) a sense of meaning, (3) a sense of interconnectedness, and (4) a sense of the beyond. The first dimension refers to the human soul or universal consciousness inherent to every person. The second dimension comprises the human experience on earth and the seeking 
for a higher truth or meaning and purpose in life. Interconnectedness describes the recognition of an encompassing energy that links all living and non-living things. The last dimension, a sense of the beyond, refers to the belief in a higher force, universal spirit, or god.

KALES (2004) suggestion aligns with considerations of WILBER (2004) who further describes spirituality as a crucial component shaping human's worldview. In his article on integrated human psychology, he further points out that spirituality is a personal experience, expressed through behavior. This connection between spirituality and human behavior has already been explored in various scientific contexts (e.g. VANDENBERGHE AND Costa PRADO 2009, BALL et al. 2001, AfSAR et al. 2016). Consequently, these implications point to a positive influence of spirituality on both general behavior and behavioral change. McGHeE AND GRANT (2008) identify four major characteristics that generally distinguish more spiritual people from less spiritual people, namely: (1) caring for others, (2) faith in higher forces, (3) inter-connectedness of matter, and (4) importance of action. The first characteristic refers to a greater concern towards the well-being of others. The second characteristic relates to the belief in greater forces. The third characteristic suggests the equal importance of all living and non-living matter. The last characteristic refers to the belief that any individual action is of great importance for the whole system. Possible implications of spirituality on food choice and dietary decisions presented by UlluWISHEWA (2014) and Subrahmanyan AND Gould (2012) who describe spirituality as a driving force for increasing altruistic values through motivating care and gratitude towards the self, others and the surrounding, and naturally reducing a materialistic and self-centered character. With an individual evolving into spirituality, these factors will find a deep grounding in the psychological disposition, shape personal values and eventually translate into behaviors that mirror these belief systems. As such, spirituality is understood as a deeply intrinsic motivation that translates into individual attitudes (SHARMA AND SHARMA 2017). The predictive strengths of spirituality then lie in this strong connection to human psychology, pointing to the fact that spirituality shapes consumption behavior on various, often very subtle, layers of consciousness with major implications for any kind of social development (CARROLL 2004). 
Given the numerous interpretations of the term spirituality, its conceptualization within consumer sciences remains a central challenge. This could explain why studies aiming at measuring the effect of spirituality on general consumer food choice and dietary behavior are still scarce. One of the first papers reflecting on spirituality in regard to consumption decisions was published by BALL and colleagues (2001) who argue that individuals with a higher level of consciousness are more likely to perceive consumption as an opportunity for spiritual growth by consuming products that add to the greater benefit of all. In a more recent study, LEE and colleagues (2016) explored spirituality of undergraduate students in the USA. The results show that students who emerged in spirituality through spiritual practice and value beliefs are more likely to express sustainable consumption behavior which is expressed in compassionate self-concepts, healthy food choices, self-confidence, volunteerism, and self-reliance. At the same time, high levels of spirituality let the participants reduce non-sustainable acts of consumption like compulsive buying and materialism. Similar supportive effects of spirituality on consumption behavior were investigated by SHARMA AND SHARMA (2017) who linked spirituality to perceived consumer effectiveness and related it to green purchase intentions. They found that the spiritual identity of consumers positively reflects on green purchase intention when mediated through perceived consumer effectiveness. A recent study of RODRIGUEZ-RAD AND RAMOS-HIDALGO (2018) explored the effects of spirituality, ethics and moral identity on consumer behavior. Here, consumers with a high level of internalized spirituality were more likely to support companies following sustainable practices.

\subsubsection{The Role of Mindfulness in Food Choice and Diet}

There are different ways to nurture personal spirituality. Commonly, ritual and different spiritual practices, like yoga, tai chi and meditation are recognized as essential tools for spiritual growth. One of these practices that emigrated from traditional Eastern belief systems into modern day life is the practice of mindfulness. Though mindfulness is commonly practiced in spirituality, people practicing mindfulness are not necessarily spiritual. Accordingly, the term 
mindfulness refers to an awareness for the present moment, including everything that happens within the individual and in the surrounding environment (ERICSON et al. 2014). According to LANGER (1989), mindfulness can be both a state and a trait. The former perceives mindfulness as a catalyst for general psychological well-being and the latter describes mindfulness in regard to personal differences based on four cognitive fields, namely (1) novelty producing, (2) novelty seeking, (3) flexibility, and (4) engagement. Both novelty producing and seeking refer to the individuals' openness to experience. Flexibility refers to the ability to question habitual norms and nomological practices. The last category describes a state of dynamic evaluation and interpretation of one's environment. If practiced regularly, mindfulness stimulates traits of compassion, empathy and care, and promotes feelings of non-reactivity, well-being and happiness (Rosenberg 2004). Furthermore, mindfulness encourages the individual to reflect on true wants, needs and desires within a light of caring compassion for the self, others, and the environment (SANGHARAKSHITA 2003, FISCHER et al. 2017).

Like spirituality, mindfulness describes a state of increased personal values ultimately aiming at inner and outer harmony (ROSENBERG 2004, SUBRAHMANYAN AND GOULD 2012). It is directly linked to the disruption of routines, congruence in terms of self-awareness, non-material values and wellbeing, as well as to pro-social behavior (FISCHER et al. 2017). If practiced over a longer period of time, mindfulness fosters altruism and will eventually translate into greater pro-environmental and pro-social behavior, making it a valuable catalyst for changes in food choice and dietary behaviors (Rosenberg 2004, Ericson et al. 2014).

In contrast to spirituality, research on mindfulness is growing rapidly in various scientific disciplines such as clinical psychology (e.g. SHAPIRO 2009, KANG AND WHITTINGHAM 2010), sociology (e.g. SCHIPPER 2012, LEE 2015), and neurosciences (e.g. ZELAZO AND LYONS 2012, TANG et al. 2015). Nevertheless, its application within the field of sustainable consumer behavior is relatively new (FISCHER et al. 2017). In regard to food choice and dietary behavior, few quantitative studies on the relationship between mindfulness and food intake 
exist. However, they show that if mindfulness is practiced for eating, it positively reflects on excessive food intake, causing people to eat less food (HENDRICKSON and Rasmussen 2013, Jordan et al. 2014, Allirot et al. 2018). Mason and colleagues (2016) studied the effect of mindfulness training on the sweets intake of obese people. They found that participants who regularly practiced mindfulness reduced their sweets intake. Furthermore, they pointed out that mindfulness practices need to anchor within the individual for several months before effects are visible. Similar outcomes were observed by JACOB and colleagues (2009) who explored the effect of mindfulness meditation on environmental sustainable behavior, measured through recycling behavior, household choice, and food practice. They found a weak but supportive relationship between mindfulness meditations and food practice. That is, practicing mindfulness frequently was associated with more sustainable food practices. In contrast, HUNECKE AND RICHTER (2019) only found weak relationships of mindfulness and environmentally relevant behavior including aspects of food choice (organic, regional, and seasonal) and diet (vegetarian). Their study recruited people with varying degrees of experience in mindfulness meditation and tested if they expressed differences in these behaviors. Differences amongst participants were studied in regard to five different mindfulness dimensions, namely: (1) observing, (2) describing, (3) acting with awareness, (4) non-judging, and (5) non-reactivity. For food choice and diet, weak positive relations were found only for the third mindfulness dimension. This implies that mindfulness research needs to be applied in a differentiated manner in order to understand how it contributes to sustainable changes in green behaviors.

\subsubsection{Study Aims}

Though much work has already been done to properly comprehend how habit and motivation affect behavior and behavioral change dynamics, recent studies have pointed to the importance of contributions that foster a deeper understanding of the relationships between personal value processes and various food consumption related behaviors (e.g. FISCHER et al. 2017). This work responds to these suggestions by exploring the relationship between different food consumption related behaviors and the intrinsic value shaping 
characteristics of spirituality and mindfulness. We believe that weak effects of mindfulness on food behaviors measured in previous studies may be due to the separation of mindfulness from its original source that is spirituality. As a trait, spirituality comprises a belief system that goes beyond the mere practice of being aware, but shapes what people believe to be true in their personal reality. Therefore, mindfulness is examined within the greater context of spirituality.

Furthermore, the present study attempts to explore spirituality and mindfulness characteristics in reflection for the broader society to account for country based differences. Therefore, India and the USA were chosen as suitable study sites to examine how spirituality and mindfulness translate into behavior when exposed to different cultural, socio-economic, and environmental contexts. The concrete study questions are: (1) Are there factorial interactions between the spirituality trait and trait mindfulness? (2) What is the effect of these factorial interactions on consumer food choice and dietary behavior?

\subsection{Methods}

\subsubsection{The Questionnaire}

Four different multi-item concepts were applied to capture the constructs of interest. The construct of food consumption behavior was evaluated using 41 items. Those items captured self-reported food choice behavior (e.g. the intentional purchase of organic food), dietary behavior (e.g. the consumption of animal based products), as well as some aspects related to personal, social, and cultural aspects of food consumption (e.g. health aspects of nutrition). Furthermore, self-reported pro-environmental behavior was evaluated using the pro-environmental behavior scale (PEBS) developed by MARKLE (2013). This scale captures environmental behavior in four dimensions, namely: (1) conservation, (2) environmental citizenship, (3) food, and (4) transportation.

Spirituality was captured through the Spiritual Attitude and Involvement List (SAIL) developed by DE JAGER MeEZENBROEK and colleagues (2012b). This scale is designed to capture spirituality as universal self-experience and encompasses seven dimensions, namely: (1) trust, (2) caring for others, (3) meaningfulness, (4) connectedness with nature, (5) acceptance, (6) spiritual 
activity, and (7) transcendent experience. Trust refers to the psychological state of belonging and connection to a higher all-encompassing force. Caring for others comprises altruistic values such as compassion and understanding. Meaningfulness reflects on the value of the individual action in context to a higher good whereas connectedness to nature emphasizes the important role of the natural environment for spiritual growth and inner harmony. The dimension of acceptance refers to faith in a higher sense. Spiritual activity takes matters of spiritual practice in consideration whereas the last dimension, transcendent experience, refers to the personal consciously experienced interactions with a higher source.

Mindfulness was captured using the Comprehensive Inventory of Mindfulness Experiences (CHIME) scale (BERGOMl et al. 2013) and captures trait mindfulness according to eight dimensions, namely: (1) inner awareness, (2) outer awareness, (3) conscious action, (4) accepting and non-judgmental attitude, (5) non-reactive and decentralized orientation, (6) open and nonavoiding attitude, (7) relativity of thoughts, and (8) insightfulness. The first two aspects of mindfulness capture the active perception and experience of personal emotional states (inner awareness) and environmental phenomena (outer awareness). Conscious action refers to the degree of present focus that an individual expresses in every day actions. Accepting and non-judgmental attitude refers to an affectionate compassion that a person shows towards the self, whereas the fifth aspect of mindfulness is concerned with the observation and conscious detachment of thoughts and feelings as they arise from an unbalanced mind. An open and non-avoiding attitude further expands on mindfulness by considering the ability of the individual to actively participate in present life actions as they appear. Relativity of thoughts pays attention to the awareness of the unsteady mind and its conscious observation. The last dimension, insightfulness, comprises the acknowledgement of the component of learning from experience.

Due to time constraints, the PEBS, the SAIL, and the CHIME were shortened in length, with two items reflecting each dimension of the corresponding scale. This resulted in eight items for the PEBS, 16 for the CHIME, and 14 for the 
SAIL. Within each dimension, items were chosen randomly. Responses were measured through five-point Likert scales ranging from 1 (never true / not at all / never / not a part of my diet) to 5 (Always true / to a high degree / always / part of my daily diet). Age, gender, education, household income and further sociodemographic information were collected in addition. After pre-testing, survey data was collected through a research panel provider in February/March 2018. The questionnaire is given in appendix B.1.

\subsubsection{Data Analysis}

The collected observations were cleaned stepwise for timing and answering patterns. To allow for chained imputations of missing values, observations with data missingness greater than $30 \%$ were excluded (as well). Multiple imputations can only be performed if data missingness is completely at random (MCAR) or at random (MAR). Little's test of MCAR was conducted according to implications given by LI (2013). The results of Little's test did not support MCAR. Since there is no statistical test available to identify MAR, the probability of a missing value association was estimated for the spirituality and mindfulness variables using a t-test. Since data missingness did not reflect on the individual variables, MAR was assumed for the dataset. Overall, data missingness was calculated at $38.8 \%$. That is, 388 observations held at least one missing value amongst the variables measured. Although data imputation is not recommended if overall missingness exceeds 30\% (AZUR et al. 2011), imputations were performed to estimate missing data. This decision was based on two reasons. Firstly, the overall missingness of the dataset was below $10 \%$ with the assumption of MAR. Secondly, there are statistical methods (e.g. ICE in Stata) that allow for solid imputations if missingness is below 50\% (ROYSTON 2009). For the imputation procedure, variables were ranked according to their degree of data missingness, starting with the variable expressing the least missing data. This was possible since Stata possesses the ability to include already imputed values into further imputation estimations. Finally, data imputation was performed using chained equations modeling (MICE) since the data set contained ordinal data. For each missing value, the number of imputed estimations was set to 20 to strengthen the estimation model. 
After the imputation procedure, the complete data set (1.012 observations) was utilized in a factor analysis in order to explore the factorial relationship between different items within the constructs under investigation. Based on the test for multivariate normality, the Iterated Principal Factor method was chosen to fit the factor model. This approach calculates measures for communality which replace the values of the original correlation matrix and are continuously reestimated until convergence is obtained (HABING 2003). The Exploratory Factor Analysis (EFA) was selected instead of the confirmatory approach, in order to explore which items naturally account together. In total, two EFA's were calculated. The first model comprised the PEBS, food choice and dietary behavior variables, and the second model the spirituality and mindfulness variables. For each EFA, the number of factors to retain was estimated using parallel analysis over ten replications. Following this approach, the appropriate factor number is determined by comparing the real Eigenvalues with simulated Eigenvalues, keeping those which are greater than the average of the simulated values (HAYTON et al. 2004). For the first EFA, seven factors were retained. For the spirituality and mindfulness variables, the parallel analysis suggested to retain four factors.

In order to clarify the results calculated through the EFA, rotations were performed for each factor model individually. Through the rotation procedure, factor items are mathematically rotated towards the factor axis which makes their interpretation easier (OSBORNE 2015). The oblique rotation was chosen since the factors are expected to correlate amongst each other. Furthermore, SCHMITT (2011) strongly recommends the use of the oblique rotation even if no correlations are assumed since it "...generally results in more realistic and more statistically sound factor structures." After the rotation procedure, items with high cross-loadings $(<0.25)$ or low item loading $(<0.35)$ were dismissed. This led to the final factor models. The first model comprised 35 items in total, expressing the shared variance across the PEBS, and the self-reported food choice and dietary behavior variables. The second model comprised 19 items and framed the factorial relationship of the spirituality and mindfulness traits. To check for internal model consistency, the Cronbach's alpha of each factor was calculated. 
Lastly, regression analysis was performed to gain insights into the relational dynamics between the two factor models. This analysis was conducted separately for each country to learn how the factor model translates the different socio-cultural and environmental influences. For each country, seven different regression models were estimated. Within the regression models, the factors designed for PEBS, food choice, and dietary behavior represented the response variable $(\mathrm{y})$ and the factors formed by items from the SAIL and CHIME represented the explanatory variables $(x)$. Control variables included within the regression analysis were age (continuous), gender (categorical), education (categorical), and income (categorical). To allow for multiple regression analysis the variables gender, education, and income were transformed into dummy variables. Each regression analysis was run including a beta construct to increase the robustness of the regression model.

\subsection{Results}

\subsubsection{Sample Description}

The surveys yielded 1.243 observations in total (621 from India and 622 from the USA). $18.6 \%$ were excluded from further data analysis processes which resulted in a final data set of 1.012 observations (482 from India and 530 from the USA). Table 3.1 provides an overview of the socio-demographic profile of the respondents sampled in India and the United States. 
Table 3.1: The summary chart of the socio-demographic profile of the respondents from India and the United States of America (USA) in spring 2018. Per annum income level: India: high = more than $1,200.000$ INR, middle $=120.000-1,200.000$ INR, low $=$ less than 120.000 INR; USA: high $=$ more than 100.000 USD, middle $=35.000-100.000$ USD, low $=$ less than 35.000 USD; $1,000,00 €$ equal $80.654,00$ INR equal $1,174,11$ USD, based on the official course rate (09.07.2018); education level: India: high = master's and higher, medium = senior school and bachelor's, low = less than senior school, USA: high = master's and higher, medium = associate's and bachelor's, low = less than college.

\begin{tabular}{|c|c|c|c|c|c|c|}
\hline \multirow[b]{2}{*}{ Demographics } & \multirow[b]{2}{*}{ Description } & \multicolumn{2}{|c|}{ India } & \multicolumn{2}{|c|}{ United States of America } & \multirow[b]{2}{*}{ Sample total [\%] } \\
\hline & & Sample [\%] & Population* [\%] & Sample [\%] & Population** [\%] & \\
\hline \multirow[t]{5}{*}{ Age } & $<26$ years & 33.0 & 45.3 & 16.4 & 34.0 & 24.3 \\
\hline & $26-35$ years & 26.8 & 26.4 & 20.2 & 12.2 & 13.1 \\
\hline & $36-55$ years & 26.0 & 14.6 & 28.3 & 25.4 & 13.1 \\
\hline & $56-65$ years & 7.1 & 7.5 & 16.4 & 13.2 & 12.0 \\
\hline & $>65$ years & 7.3 & 6.2 & 18.7 & 15.2 & 13.2 \\
\hline \multirow[t]{2}{*}{ Gender } & Female & 50.8 & 48.5 & 50.4 & 50.8 & 50.6 \\
\hline & Male & 49.2 & 51.5 & 49.6 & 49.2 & 49.4 \\
\hline \multirow[t]{3}{*}{ Education } & High & 81.3 & 6.7 & 16.0 & 13.0 & 47.1 \\
\hline & Middle & 12.9 & 6.7 & 60.4 & 32.1 & 37.7 \\
\hline & Low & 5.8 & 86.6 & 23.6 & 54.9 & 14.1 \\
\hline \multirow[t]{3}{*}{ Income $* * *$} & High & 9.8 & 0.2 & 25.1 & 27.6 & 17.8 \\
\hline & Middle & 52.5 & 32.2 & 44.2 & 42.2 & 48.1 \\
\hline & Low & 25.1 & 67.6 & 29.2 & 30.2 & 27.3 \\
\hline Sample size & & 482 & & 530 & & 1012 \\
\hline
\end{tabular}

* Population statistics drawn from the Office of the Registrar General \& Census COMMISSIONER, India (2001) and STATISTA (2015) data.

** Population statistics drawn from STATISTICALATLAS (2018)

${ }^{* * *}$ Income level shows response rates of below $100 \%$

Both country samples properly correspond to the population shares reported for gender and age classes of 56 and older. Though quotas were set for the demographic variables measured, restrictions on age, education and income needed to be relaxed during data collection process in order to reach the observation number desired. Therefore, our sample is biased towards Indian consumers with high education levels and incomes greater than 120.000 INR (categories "high" and "middle"). In regard to education, this was also true for the US observations collected. In both samples, consumers younger than 26 years are underrepresented.

\subsubsection{Factor Structures derived from the EFA}

Table 3.2 provides an overview of the factors extracted through the EFA procedure. 
Table 3.2: Summary of the results of the two Exploratory Factor Analyses performed for selfreported food choice and dietary behavior $(n=1012)$ and trait spirituality and trait mindfulness. Data was collected through online surveys from India and the United States in spring 2018.

\begin{tabular}{lccc}
\hline \multicolumn{1}{c}{ Factors derived } & \multicolumn{3}{c}{ Exploratory factor analysis } \\
Food choice and diet ( $\mathbf{n}=1012)$ & Number of items & Item loading range & Cronbach's alpha \\
\hline Informed Food Choice (IFC) & 8 & $0.53-0.85$ & 0.902 \\
Pro-Environmental Behavior (PEB) & 5 & $0.37-0.58$ & 0.674 \\
Dietary Awareness (DA) & 5 & $0.46-0.65$ & 0.699 \\
Convenience Diet (CD) & 2 & $0.55-0,72$ & 0.582 \\
Animal-Protein based Food (APF) & 7 & $-0.43-0.84$ & 0.822 \\
Plant-Protein based Food (PPF) & 4 & $0.44-0.57$ & 0.664 \\
Fruits and Vegetables (FV) & 2 & $0.85-0.85$ & 0.881 \\
Spirituality and mindfulness ( $\mathbf{n}=1012)$ & & & \\
Metaphysical spirituality (SPIRITI) & 5 & $0.63-0.85$ & 0.877 \\
Physical spirituality (SPIRITII) & 5 & $0.41-0.74$ & 0.745 \\
Affirmative mindset (MINDI) & 6 & $0.44-0.68$ & 0.760 \\
Scattered mindset (MIND II) & 4 & $0.46-0.61$ & 0.598 \\
\hline
\end{tabular}

For the behavioral variables, the EFA of the merged data sets resulted in seven factors, each corresponding to a different aspect of food choice or dietary behavior. The factors identified were: (1) informed food choice, (2) proenvironmental behavior, (3) dietary awareness, (4) convenience diet, (5) animal-protein based food, (6) plant-protein based food, and (7) fruits and vegetables.

Informed food choice (IFC) emerges from an item pool that reflects a high degree of information based responsible food consumption behavior. The eight items feeding into this factor are mainly concerned with the intentional purchase of certified and labeled food produce (e.g. organic, fair-trade, and animal welfare characteristics) and the degree of wholesomeness of a produce. Within the behavioral EFA, IFC holds the highest internal consistency ( $\alpha=0.902)$. The second factor, PEB, is formed by five items of the PEB questionnaire and captures environmental behavior not only related to food choice and diet but also other facets of consumption like means of transportation or energy consumption behavior. The third factor is framed around dietary diversity and well-being / health (e.g. "For my meals, I alternate between different food groups" and "I consciously choose food that is low in sugar"). Furthermore, the 
item loadings also suggest sensitivity for personal food consumption behaviors (e.g. "I only eat when I am hungry"). For that reason, this factor is named Dietary Awareness (DA). The fourth and the fifth factor depict the Westernization of diets. Factor four (convenience diet) refers to the consumption of convenience food and highly processed foods (e.g. refined sugar). It is fed by two items and has the lowest internal consistency amongst the seven factors calculated $(\alpha=0.582)$. Factor five (animal-protein based foods) is concerned with the consumption of different kinds of meats e.g. red meat and processed meat and other animal based products (e.g. eggs and/or seafood). The last two factors concern plant derived foods. The factor plantprotein based food comprises foods high in protein like legumes, nuts, seeds and cereals. The last factor, fruits and vegetables, includes two items measuring unprocessed fruits and vegetables.

The items of the predictor variables derived from two constructs, namely mindfulness (CHIME) and spirituality (SAIL). The EFA procedure, however, resulted in four factors. Two of those factors (factor two and three) combined items from both constructs to form a factor structure. The first and last factors were fed by items from the CHIME or the SAIL only. Table 3.3 provides a detailed overview of the items loading each factor. 
Table 3.3: List of the item loadings of the four factors (SPIRIT I, SPIRIT II, MIND I, and MIND II) derived during EFA procedure for trait spirituality and trait mindfulness based on 1012 observations. Data was collected through online surveys from India and the United States in spring 2018.

\begin{tabular}{|c|c|c|c|c|}
\hline \multirow[b]{2}{*}{ Statement } & \multicolumn{4}{|c|}{ Factor loading } \\
\hline & SPIRIT I & SPIRIT II & MIND I & MIND II \\
\hline I consider myself a religious person & 0.847 & & & \\
\hline I consider myself a spiritual person & 0.782 & & & \\
\hline There is a God or higher power in my life that gives me guidance* & 0.738 & & & \\
\hline $\begin{array}{l}\text { I have had experiences in which I seemed to merge with a power } \\
\text { greater than myself* }\end{array}$ & 0.709 & & & \\
\hline I meditate or pray, or take time in other ways to find inner peace * & 0.635 & & & \\
\hline When I am in nature, I feel a strong sense of connection* & & 0.736 & & \\
\hline The beauty of nature moves me* & & 0.712 & & \\
\hline It is important to me that I can do things for others* & & 0.554 & & \\
\hline I am aware of the fact that each life has its own tragedy* & & 0.525 & & \\
\hline $\begin{array}{l}\text { I consciously notice sounds around me, like birds chirping or cars } \\
\text { passing by }\end{array}$ & & 0.411 & & \\
\hline $\begin{array}{l}\text { I notice my thoughts and feelings, yet I am able to look at them from a } \\
\text { distance** }\end{array}$ & & & 0.682 & \\
\hline $\begin{array}{l}\text { I guide myself with loving kindness through the ups and downs of } \\
\text { life** }\end{array}$ & & & 0.636 & \\
\hline $\begin{array}{l}\text { When I am tangled up in stressful thoughts and feelings, I notice this } \\
\text { quickly and can easily dissociate myself from them *. }\end{array}$ & & & 0.506 & \\
\hline In difficult times, I maintain my inner peace* & & & 0.500 & \\
\hline $\begin{array}{l}\text { When I notice that I have made things more complicated than they } \\
\text { really are, I can smile about it** }\end{array}$ & & & 0.498 & \\
\hline I approach the world with trust* & & & 0.438 & \\
\hline $\begin{array}{l}\text { In the everyday life, loften get distracted by memories, images or } \\
\text { dreams** }\end{array}$ & & & & 0.605 \\
\hline $\begin{array}{l}\text { While reading, I often have to re-read sections because I get } \\
\text { distracted by my thoughts** }\end{array}$ & & & & 0.572 \\
\hline I judge myself harshly when I make a mistake** & & & & 0.476 \\
\hline $\begin{array}{l}\text { I am aware of the fact that my judgments about situations and } \\
\text { persons can change easily** }\end{array}$ & & & & 0.463 \\
\hline Cronbach's alpha & 0.877 & 0.745 & 0.760 & 0.598 \\
\hline
\end{tabular}

* Items derived from the Spiritual Attitude and Involvement List (SAIL; DE JAGER MEeZenBroek et al. 2012b).

** Items derived from Comprehensive Inventory of Mindfulness Experiences (CHIME; BERGOMI et al. 2013).

SPIRIT I depicts spirituality in close relationship to the supernatural and/or religious belief. Out of the five factors loading into SPIRIT I, three suggest the belief in a force greater than the self (e.g. "There is a God or higher power in my life that gives me guidance"). The item with the lowest internal factor loading ("I meditate or pray, or take time in other ways to find inner peace") indicates the integration of some kind of ritualistic behavior. Here, the individual creates space in the everyday life to emerge into spiritual practice. It should be noticed that the items "I consider myself a spiritual person" and "I consider myself a 
religious person" are not part of the SAIL questionnaire but were added additionally. The term spirituality is sometimes associated with one's relationship to God (VAUGHAN 2002) which supports the religious connotation already emphasized by other items of this factor. Amongst the spirituality and mindfulness factors, SPIRIT I reveals the highest internal consistency $(\alpha=$ $0.877)$.

In contrast to SPIRIT I where the self is driven towards a higher force or the supernatural, SPIRIT II is concerned with the earthly matters of spirituality. It reflects spirituality as perceived, experienced and expressed in daily routine which reflects on every interaction with others or the environment (e.g. "It is important for me that I can do things for others" or "the beauty of nature moves $m e ")$. When observing the item structure of SPIRIT II, it is further noticeable that several items point to individuals with integrated emotional states comprising compassion (e.g. "I am aware of the fact that every life has its own tragedy") and belonging (e.g. When I am in nature, I feel a strong sense of connection"). For this factor, all items but the last derive from the spirituality scale (SAIL).

Both the third and fourth factor determined through the EFA procedure are framed around self-perception in daily experience. Yet, they reflect this from two opposing perspectives. MIND I depicts a strong responsive compassion for the self (e.g. "I guide myself with loving kindness through the ups and downs of life" and "When I notice that I've made things more complicated than they really are, I can smile about it"). It points to an attitude that responds supportive, allowing to experience failure. It further describes a mindset that is aware and present with emotions concerning the self (e.g. "I notice my thoughts and feelings, yet I am able to look at them form a distance"). The items "In difficult times, I maintain my inner peace" and "I approach the world with trust" originate from the spirituality construct (SAIL) further reinforcing a positive thought structure of the individual.

In contrast, MIND II refers to a rather scattered mindset. It is shaped by four items of which two refer to a state of mental absence and imbalance ("In the everyday life, I often get distracted by memories, images, or dreams" and "While reading, I often have to re-read sections because I get distracted by my 
thoughts"). MIND II also carries a connotation to self-criticism and nonintegrated judgment (e.g. "I judge myself harshly when I make mistakes"). Among the intentional factors Mind II has the lowest but still acceptable internal reliability $(\alpha=0.598)$.

\subsubsection{The Effect of Spirituality and Mindfulness on Food Choice and Diet}

For each country, multiple regression analysis was performed to gain insights on the relationship between the seven food choice and dietary behavior factors and the four spirituality and mindfulness factors derived from the EFA. A partial overview of the results is given in tables 3.4 and 3.5. A complete overview including the four socio-demographic control variables, age, gender, education, and income is given in chapter 3.7. 


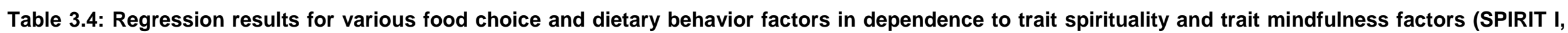

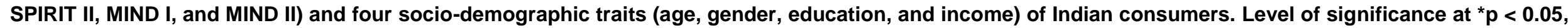

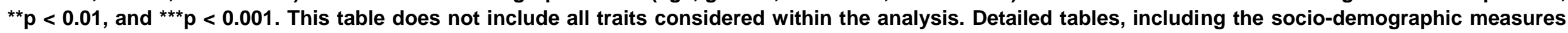
are given in chapter 3.7.

India $(\mathrm{n}=482)$

\begin{tabular}{|c|c|c|c|c|c|c|c|c|c|c|c|c|c|c|c|c|c|c|c|c|c|}
\hline \multirow[t]{2}{*}{ Factor } & \multicolumn{3}{|c|}{ Informed food choice } & \multicolumn{3}{|c|}{ Pro-environmental behavior } & \multicolumn{3}{|c|}{ Dietary awareness } & \multicolumn{3}{|c|}{ Animal-protein based diet } & \multicolumn{3}{|c|}{ Convenience diet } & \multicolumn{3}{|c|}{ Plant-protein based diet } & \multicolumn{3}{|c|}{ Fruits and vegetables } \\
\hline & beta & Std. Err. & $\mathbf{t}$ & beta & Std. Err. & $\mathbf{t}$ & beta & Std. Err. & $\mathbf{t}$ & beta & Std. Err. & $\mathbf{t}$ & beta & Std. Err. & $\mathbf{t}$ & beta & Std. Err. & $\mathbf{t}$ & beta & Std. Err. & $\mathbf{T}$ \\
\hline (Constant) & - & 0.026 & 10.55 & - & 0.018 & 57.10 & - & 0.023 & 3.74 & - & 0.030 & -64.14 & - & 0.020 & -63.77 & - & 0.029 & -0.30 & - & 0.023 & 7.68 \\
\hline SPIRIT I & 0.14 & 0.008 & 19.51 & 0.11 & 0.005 & 15.45 & 0.03 & 0.007 & -5.37 & 0.14 & 0.009 & 19.79 & 0.28 & 0.006 & 43.22 & -0.15 & 0.009 & -20.07 & -0.11 & 0.007 & -16.26 \\
\hline SPIRIT II & -0.27 & 0.009 & -18.14 & -0.17 & 0.006 & -19.41 & -0.09 & 0.007 & -11.82 & -0.39 & 0.010 & -40.94 & -0.49 & 0.007 & -55.41 & 0.01 & 0.010 & 0.94 & 0.48 & 0.008 & 53.92 \\
\hline MIND I & 0.22 & 0.010 & 23.48 & 0.41 & 0.007 & 45.29 & 0.70 & 0.009 & 91.76 & 0.24 & 0.011 & 25.31 & 0.13 & 0.008 & 14.95 & 0.02 & 0.011 & 2.02 & -0.12 & 0.009 & -13.64 \\
\hline MIND II & 0.19 & 0.007 & 28.77 & 0.15 & 0.005 & 24.59 & -0.01 & 0.006 & -2.46 & 0.11 & 0.008 & 17.25 & 0.20 & 0.006 & 34.34 & 0.14 & 0.008 & 21.52 & 0.19 & 0.006 & 32.87 \\
\hline adjusted $R^{2}$ & & 0.18 & & & 0.27 & & & 0.47 & & & 0.17 & & & 0.30 & & & 0.06 & & & 0.29 & \\
\hline
\end{tabular}

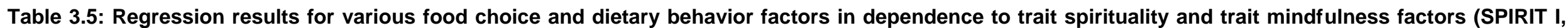
SPIRIT II, MIND I, and MIND II) and four socio-demographic traits (age, gender, education, and income) of US consumers. Level of significance at ${ }^{*} p<0.05$, ** $p$

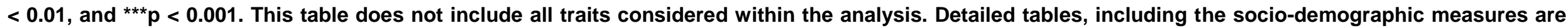
given in chapter 3.7 .

USA $(n=530)$

\begin{tabular}{|c|c|c|c|c|c|c|c|c|c|c|c|c|c|c|c|c|c|c|c|c|c|}
\hline \multirow[t]{2}{*}{ Factor } & \multicolumn{3}{|c|}{ Informed food choice } & \multicolumn{3}{|c|}{ Pro-environmental behavior } & \multicolumn{3}{|c|}{ Dietary awareness } & \multicolumn{3}{|c|}{ Animal-protein based diet } & \multicolumn{3}{|c|}{ Convenience diet } & \multicolumn{3}{|c|}{ Plant protein based diet } & \multicolumn{3}{|c|}{ Fruits and vegetables } \\
\hline & beta & Std. Err. & $\mathbf{t}$ & beta & Std. Err. & $\mathbf{t}$ & beta & Std. Err. & $\mathbf{t}$ & beta & Std. Err. & $\mathbf{t}$ & beta & Std. Err. & $\mathbf{t}$ & beta & Std. Err. & $\mathbf{t}$ & beta & Std. Err. & $\mathbf{t}$ \\
\hline (Constant) & - & 0.021 & -31.58 & - & 0.020 & -66.84 & - & 0.016 & -3.40 & - & 0.018 & -3.96 & & 0.024 & 0.26 & - & 0.022 & -14.63 & - & 0.019 & -13.65 \\
\hline SPIRIT I & 0.14 & 0.005 & 25.13 & 0.10 & 0.005 & 17.41 & -0.15 & 0.004 & -30.11 & -0.01 & 0.004 & -0.97 & 0.01 & 0.006 & 1.41 & -0.03 & 0.005 & -4.99 & 0.02 & 0.005 & 3.84 \\
\hline SPIRIT II & 0.45 & 0.008 & 65.64 & -0.01 & 0.007 & -1.23 & 0.35 & 0.006 & 54.81 & 0.14 & 0.007 & 18.32 & -0.38 & 0.009 & -50.45 & 0.45 & 0.008 & 60.36 & 0.34 & 0.008 & 44.73 \\
\hline MIND I & 0.02 & 0.007 & 2.98 & 0.33 & 0.007 & 49.05 & 0.36 & 0.005 & 60.09 & -0.01 & 0.006 & -0.88 & 0.16 & 0.008 & 22.71 & -0.14 & 0.007 & -19.41 & 0.02 & 0.006 & 2.99 \\
\hline MIND II & 0.01 & 0.007 & 2.07 & 0.15 & 0.006 & 25.95 & -0.01 & 0.005 & -3.57 & 0.13 & 0.006 & 21.35 & 0.19 & 0.007 & 31.61 & 0.03 & 0.006 & 5.66 & 0.08 & 0.006 & 12.72 \\
\hline adjusted $R^{2}$ & & 0.31 & & & 0.27 & & & 0.40 & & & 0.13 & & & 0.16 & & & 0.19 & & & 0.20 & \\
\hline
\end{tabular}




\subsubsection{Data Set: India}

For the Indian dataset, the attribute of earth-centered spirituality (SPIRIT II) and an affirmative mindset (MIND I) revealed to be strong predictors for the estimated (un-)sustainable food consumption behaviors. Consumers expressing a high degree of SPIRIT II were less likely to consume high amounts of convenience products $(-0.49, p<0.01)$ and animal based foods $(-0.39, p<0.01)$, but more likely to consume unprocessed fruits and vegetables $(0.48, p<0.01)$. Mind I was strongly positively related to dietary awareness $(0.70, p<0.01)$, proenvironmental behavior $(0.41, p<0.01)$, and informed food choice $(0.22$, $\mathrm{p}<0.01)$. That is, an affirmative mindset greatly increased the likelihood of expressing sustainability behaviors. In contrast, respondents with a scattered mindset related comparably weak with dietary awareness $(-0.01, p<0.01)$, and pro-environmental behavior $(0.15, p<0.01)$.

The socio-demographic variables age, gender, education, and income are not given in the table above, but were part of the estimation model. Among these four variables income levels revealed to be comparably strong predictors. Respondents earning more than 1,200.000 INR per annum were more likely to consume animal-protein based foods $(0.24, p<0.01)$ and make informed food choices $(0.25, p<0.01)$ when compared to respondents earning 120.000 INR per annum or less. Younger respondents were more likely to consume animalprotein based foods $(0.22, p<0.01)$ when compared to older respondents. The education level formed the strongest relationship with fruit and vegetable consumption. Respondents holding at least a Bachelor's degree were more likely to consume unprocessed fruits and vegetables (Senior school or Bachelor's degree: 0.14, $p<0.01$; Master's degree or higher: $0.18, p<0.01$ ) than respondents with lower education. Gender related weakly to all behaviors evaluated. Yet, there was a tendency of male respondents to eat more animalprotein based foods $(0.07, p<0.01)$ and convenience foods $(0.02, p<0.01)$ but lesser amounts of unprocessed fruits and vegetables $(-0.12, p<0.01)$ when compared to females. 


\subsubsection{Data Set: United States of America}

As for the Indian sample, within the American dataset SPIRIT II and MIND I were also valuable predictors. In particular, SPIRIT II related strongly to informed food choice $(0.45, p<0.01)$, convenience foods $(-0.38, p<0.01)$, dietary awareness $(0.35, p<0.01)$, fruit and vegetable consumption $(0.34, p<0.01)$, and plant protein based foods $(0.34, p<0.01)$. MIND I had a comparably large influence on dietary awareness $(0.36, p<0.01)$ and pro-environmental behavior (0.33, p<0.01). SPIRIT I showed relatively week relationships towards all outcome variables estimated. Additionally, it is the only predictor within the American subsample that has a comparably negative relationship with dietary awareness $(-0.15, p<0.01)$. This implies that with increasing metaphysical spirituality, dietary awareness decreases within the American sample. The influence of MIND II on the (un-)sustainable behavior variables is comparable to the estimates of the Indian regression model.

Age was a relatively strong predictor variable indicating that younger respondents were more likely to express greater pro-environmental behavior (0.35, $\mathrm{p}<0.01)$, informed food choice $(0.23, \mathrm{p}<0.01)$ and plant-protein based dietary behavior $(0.12, \mathrm{p}<0.01)$. Moreover, income levels revealed to be valuable predictors in particular for the dietary behavior variables. Respondents of higher income classes consumed more animal-protein based foods (35.000100.000 USD/year: $0.26, p<0.01$; $>100.000$ USD/year: $0.17, p<0.01)$ but also more plant-protein based foods (35.000-100.000 USD/year: 0.08, $\mathrm{p}<0.01$; $>100.000$ USD/year: $0.30, p<0.01)$. Similar to the Indian dataset, males tended to eat more animal-protein based foods $(0.24, p<0.01)$ and convenience foods $(0.13, p<0.01)$ and expressed a lower degree of dietary awareness $(-0.10$, $p<0.01)$ and informed food choice behavior $(-0.10, p<0.01)$. Respondents of higher education consumed comparably lower amounts of animal-protein based foods (Bachelor's or associates: $-0.08, p<0.01$; Master's or higher: -0.12 , $\mathrm{p}<0.01$ ) but more unprocessed fruits and vegetables (Bachelor's or associates: $0.04, \quad p<0.01$; Master's or higher: $0.13, p<0.01$ ) than lower educated respondents. 


\subsection{Discussion}

This study aimed at exploring the application of spirituality and mindfulness constructs and their influence on consumer food choice and dietary behavior. In the following section the results are discussed, first, by looking at the conceptualized structures, and second, by discussing their effect in

\subsubsection{The Value of Spirituality and Mindfulness for Sustainable Food Consumption Research}

When fed into the EFA both constructs, trait spirituality (SAIL) and trait mindfulness (CHIME), remained largely independent from each other. However, during the analysis process the individual items formed four independent factors (SPIRIT I, SPIRIT II, MIND I, and MIND II) describing different states of experienced and perceived responsibility. The first two factors SPIRIT I and SPIRIT II were mostly obtained from the spirituality construct as well as from two additional questions added by the authors. Overall, the findings indicate the independence of spirituality and mindfulness as measures in their scientific application. A clear distinction between spirituality and mindfulness was also found by LEIGH and colleagues (2005), who studied the implications of spirituality and mindfulness on substance abuse. They concluded that mindfulness has indeed emerged as an independent activity that does not necessarily comprise spiritual belief. While our study reinforces these implications, central questions on the possible interactions of spirituality and mindfulness on each other, e.g. as mediator or moderator for behavior or behavior change, remain unexplored within the framework of this study.

SPIRIT I characterizes a supernatural understanding of spirituality whereas SPIRIT II describes spirituality as embedded in daily experience. A similar differentiation was made by ALLPORT (1963) who described the behavioral implications of religiousness for mental health. He suggested conceptualizing religion as motivation that is either extrinsically or intrinsically orientated. Extrinsically motivated people will turn to authority, which is expressed through a higher force but also through the active participation in religious practices when in need for guidance. Intrinsically motivated people, however, will turn to the self to find direction. Our results support this distinction by differentiating 
spirituality in two similar categories. Therefore, we argue that it may be more effective to base measures of trait spirituality in consumer sciences on fewer factors to capture the essential mechanism of spirituality in regard to consumption behavior. The items framing such a construct, however, need to be well selected in order to capture the holistic concept of spirituality sufficiently. An important differentiation between SPIRIT I and SPIRIT II is the aspect of experienced and perceived responsibility. In SPIRIT I, responsibility is decentralized from the self. This, however, implies a codependency where the individual action is limited in scope as it is objected to a more powerful source. This aspect is the major delineation to SPIRIT II, where full responsibility is given to the self. In this regard, the construct of spirituality captured in the framework of this study reminds of the concept of perceived consumer effectiveness (PCE) introduced by KINNEAR and colleagues (1974). PCE aims at capturing the degree to which people belief in the power of an individual consumption act in order to implement large-scale changes. Though there are similarities between the investigated factor structure of SPIRIT II and the PCE concept, we argue that both constructs are independent from each other. While actions in PCE are motivated upon the belief that the individual decision will make a difference (RICE 2006), in the spirituality construct it seems that the behavior will be undertaken in any case even though the effect on the larger environment is unclear or minimal. Hence, spirituality, especially physical spirituality (SPIRIT II), may delineate a different motivational driver for human behavior than PCE.

This implication may also hold true for the third and the forth factors (MIND I and MIND II) which both derived from the CHIME questionnaire for mindfulness. No study was found that measured mindfulness from the perspective of opposing mindsets of either being lost in thought and judgment or of focused awareness for the emotional dynamics within and outside the self. In regard to mindsets, issues of sustainable behavior are mostly investigated in terms of specific positive or negative opinions towards a product or processing method which is closely related to personal moral and ethical believes (e.g. Lu et al. 2015, Chen AND Moosmayer 2020). The structure discovered in the framework of this study, however, suggests looking at those mindsets from a more 
fundamental viewpoint which is the cognitive presence and its mental translation to perceived experience. Further elaboration on this construct structure could prove valuable, since an affirmative mindset (MIND I) was found to be a strong predictor for several of the food choice and dietary behaviors evaluated.

\subsubsection{Effects of SPIRIT I, SPIRIT II, MIND I, and MIND II on Consumer Food Choice and Diet}

Though almost all predictors significantly influence the evaluated food choice and dietary behavior variables, it becomes clear that the different plains of spirituality and mindfulness affect different behaviors.

Metaphysical spirituality (SPIRIT I) somehow relates to religion which is, similar to spirituality, also a search for the sacred but commonly contains an institutional component based on rules, regulations, and traditions (ZINNBAUER et al. 1997). Although the item structure of SPIRIT I indicates religious orientation, predictive dynamics of SPIRIT I between the country sets do not reinforce a close affiliation between these two aspects. Especially, the relatively strong increase in animal-protein based foods in India and the similar predictive outcomes for the pro-environmental behavior variable in both country samples do not support this assumption but reinforces the already established delineation between spirituality and religious orientation.

Physical spirituality (SPIRIT II) reduces the consumption of convenience products like soft drinks and ready-to eat products and increases the intake of unprocessed fruits and vegetables in both countries. Within the Indian sample, SPIRIT II also decreases the consumption of animal-protein based foods, while in the American sample informed food choice and plant-protein based foods are further behaviors positively related to SPIRIT II. These results align with the indications of RODRIGUEZ-RAD AND RAMOS-HIDALGO (2018) who suggest that more integrated forms of spirituality lead to a greater degree of sustainable behavior as they bridge moral identity into action. Hence, our findings point to the importance of physical spirituality within the context of sustainable social transitions. As the consumer is considered to be the major driving force for changes in diet (REISCH et al. 2013) a thorough understanding on physical 
spirituality as motivational driver for sustainability behavior may be a key feature for successful implementation of interventions in this regard. However, it should be noted that across samples, pro-environmental behavior (PEB) either decreased (India) or remained relatively unaffected (USA) by the SPIRIT II predictor which is different from current findings emphasizing the supportive effect of spirituality on pro-environmental behavior (GARFIELD et al. 2014, AFSAR et al. 2016). The negative relationship of SPIRIT II and PEB within the Indian sample might be explained through the items used to capture PEB. In this study, the factor measuring pro-environmental behavior comprised three out of four available domains, namely: environmental citizenship, means of transportation, and consumption of animal-based food products. However, due to differences in cultural perceptions and possibilities e.g. using a bicycle to get to work, this factor might mirror PEB from a perspective that is less suitable within the cultural context of this study.

An affirmative mindset (MIND I) leads to greater pro-environmental behavior (PEB) and dietary awareness in both sample sets. Additionally, within the Indian sample, MIND I supports informed food choice. These implications were also reported by several other researchers who studied the effects of mindfulness on PEB (BARBARo And Pickett 2016, TANG et al. 2017, PANNo et al. 2018), dietary awareness, and food choice (JORDAN et al. 2014, MASON et al. 2016, ALLIROT et al. 2018). However, when comparing the results from this study with similar results it should be considered that mindfulness was not measured using a scoring system but through the calculation of factor variables. For MIND I, extracted item structures derived from the domains of decentering/non-reactive orientation, acceptance/non-judgment/compassionate attitude and insightful understanding. Furthermore, this factor was fed by two items deriving of the SAIL questionnaire for spirituality which corresponded to the domain of trust. This implies that, certain facets of mindfulness relate stronger to PEB and dietary awareness than others and are less significant for the expression of other food consumption behaviors. Though a scattered mindset (MIND II) showed similar directional dynamics when compared to the effect of MIND I, its predictive strength is weaker in many cases. For both country samples this is particularly true for informed food choice, dietary awareness and PEB. In turn, 
MIND II had a greater effect on the intake of convenience food and animalprotein based foods (USA only) when compared to MIND I. These findings show how important a clear delineation within the construct of mindfulness is for exploring the potential of mindfulness practices as driver for sustainable development in food practices. It further reflects on the value of a sound understanding of both spirituality and mindfulness within the sustainable research environment. This is especially relevant, since MIND I and MIND II were the only estimators among the four main predictor variables that followed similar patterns across country data sets. The existence of a universal characteristic reliably predicting food consumption behaviors across cultural and regulative circumstances may be of great value for the successful implementation of long-lasting sustainability practices into the broader societal context.

In this regard, a further differentiation of mindfulness is also necessary with respect to healthy dietary behaviors as measured through the self-reported intake of unprocessed fruits and vegetables and plant-protein based foods. Across country samples, the likelihood of these behaviors was more strongly related with a scattered mindset and not with an affirmative mind. This contradicts the general understanding of mindfulness as mediator for healthy eating patterns (FISCHER et al. 2017) and, hence, further scientific elaboration is required to fully understand the dynamics of mindset structures on consumption behavior. A possible explanation for this may be found in traditional spirituality where mindfulness, as spiritual practice, is used for personal development towards enlightenment. It appears that mindfulness is used as a tool to counterbalance the shortcomings of human psyche and emotion. This implies that mindfulness still needs to be practiced and thus, the individual is in an unbalanced state of general being which may be expressed in some unbalanced or unsustainable behavior towards the self and the environment. However, this also indicates that a greater integration of mindfulness into the personal mindset eventually leads to a balanced consumption behavior. 


\subsection{Conclusion}

As to date, this is the first approach that quantitatively measures spiritual and mindfulness traits and their influence on food choice and dietary behavior based on a sample drawn from the general society. Our results reinforce the independence of trait spirituality and trait mindfulness as measured constructs and reveal a new perspective for the interpretation of both constructs. Within each two sub-dimensions, spirituality and mindfulness traits proved conclusive and clear in regard to their structural interpretation which is highly valuable especially if both are used as side constructs in questionnaires.

When related to food choice and dietary behavior this study proposes that specific factorial characteristics of both constructs, but especially of mindfulness, have a strong effect on sustainability relevant food consumption behaviors like pro-environmental behavior, dietary awareness, and informed food choice behavior. Hence, spiritual consciousness and mindfulness practices hold the potential to increase long-term sustainability with respect to consumer food choice and dietary behavior. However, ambiguity exists concerning important dietary sustainability measures, e.g. the consumption of animalprotein based foods. Further research would therefore benefit from a deeper scientific exploration of spirituality and mindfulness traits and specific dietary outcomes.

Our implications are important for future research focusing on the integration of spirituality and mindfulness concepts into food consumption research. Furthermore, the insights of this study support companies who aim at launching spirituality or awareness campaigns to increase their sales for sustainable food products. 


\subsection{Additional Tables}

Table 3.6: Regression results for various food choice and dietary behavior factors in dependence to trait spirituality and trait mindfulness factors (SPIRIT I, SPIRIT II, MIND I, and MIND II) and four socio-demographic traits (age, gender, education, and income) of Indian consumers. Level of significance at ${ }^{*} p<0.05,{ }^{\star *} p<0.01$, and ${ }^{\star * *} p<0.001$.

\begin{tabular}{|c|c|c|c|c|c|c|c|c|c|c|c|c|}
\hline India $(n=482)$ & & & & & & & & & & & & \\
\hline \multirow[t]{2}{*}{ Trait } & \multicolumn{3}{|c|}{ IFC } & \multicolumn{3}{|c|}{ APD } & \multicolumn{3}{|c|}{ DA } & \multicolumn{3}{|c|}{ PEB } \\
\hline & beta & $\begin{array}{l}\text { Std. } \\
\text { Err. }\end{array}$ & $t$ & beta & $\begin{array}{l}\text { Std. } \\
\text { Err. }\end{array}$ & $t$ & beta & $\begin{array}{l}\text { Std. } \\
\text { Err. }\end{array}$ & $\mathbf{t}$ & beta & $\begin{array}{l}\text { Std. } \\
\text { Err. }\end{array}$ & $\mathbf{t}$ \\
\hline (Constant) & & 0.026 & 10.55 & & 0.030 & $\begin{array}{c}- \\
46.14\end{array}$ & & 0.023 & 3.74 & & 0.018 & 57.10 \\
\hline SPIRITI & 0.14 & 0.009 & 19.51 & 0.14 & 0.009 & 19.79 & 0.04 & 0.007 & 5.37 & 0.10 & 0.005 & 15.45 \\
\hline SPIRITII & 0.17 & 0.009 & 18.14 & 0.39 & 0.010 & 40.94 & 0.09 & 0.008 & 11.82 & 0.17 & 0.006 & 19.41 \\
\hline MINDI & 0.22 & 0.010 & 23.48 & 0.24 & 0.011 & 25.31 & 0.78 & 0.009 & 91.76 & 0.41 & 0.007 & 45.29 \\
\hline MINDII & 0.19 & 0.007 & 28.77 & 0.11 & 0.008 & 17.25 & $\begin{array}{c}- \\
0.02\end{array}$ & 0.006 & -2.46 & 0.15 & 0.005 & 24.59 \\
\hline year & $\overline{0} 05$ & 0.003 & -9.02 & 0.22 & 0.000 & 38.83 & $\overline{-}$ & 0.000 & -4.45 & $\overline{0}-\overline{22}$ & 0.000 & $\begin{array}{c}- \\
39.96\end{array}$ \\
\hline gender & $\overline{-} .06$ & 0.010 & $\begin{array}{c}- \\
10.15\end{array}$ & 0.07 & 0.012 & 13.65 & $\begin{array}{c}- \\
0.07\end{array}$ & 0.009 & $\begin{array}{c}- \\
15.23\end{array}$ & $\begin{array}{c}- \\
0.04\end{array}$ & 0.007 & -7.19 \\
\hline \multicolumn{13}{|l|}{ Education level } \\
\hline Bachelor's & $\overline{-}-\overline{05}$ & 0.014 & -6.89 & $\begin{array}{c}- \\
0.12\end{array}$ & 0.016 & $\begin{array}{c}- \\
16.09\end{array}$ & $\begin{array}{c}- \\
0.12\end{array}$ & 0.012 & $\begin{array}{c}- \\
19.82\end{array}$ & $\begin{array}{c}- \\
0.01\end{array}$ & 0.010 & -1.10 \\
\hline Master's or higher & 0.08 & 0.016 & 10.45 & $\begin{array}{c}- \\
0.05\end{array}$ & 0.018 & -6.86 & $\begin{array}{c}- \\
0.10\end{array}$ & 0.013 & $\begin{array}{c}- \\
16.08\end{array}$ & $\begin{array}{c}- \\
0.07\end{array}$ & 0.011 & -9.05 \\
\hline \multicolumn{13}{|l|}{$\begin{array}{l}\text { Income level } \\
\text { (INR/year) }\end{array}$} \\
\hline $120.000-1,200.000$ & 0.13 & 0.012 & 20.76 & 0.07 & 0.136 & 11.94 & 0.14 & 0.010 & 26.80 & 0.07 & 0.008 & 11.09 \\
\hline$>1,200.000$ & 0.25 & 0.020 & 37.76 & 0.24 & 0.022 & 37.16 & 0.02 & 0.017 & 5.31 & $\begin{array}{c}- \\
0.16\end{array}$ & 0.013 & $\begin{array}{c}- \\
26.49\end{array}$ \\
\hline adjusted $\mathrm{R}^{2}$ & & 0.18 & & & 0.17 & & & 0.47 & & & 0.27 & \\
\hline
\end{tabular}

India $(n=482)$

\begin{tabular}{|c|c|c|c|c|c|c|c|c|c|}
\hline \multirow[t]{2}{*}{ Trait } & \multicolumn{3}{|c|}{ FV } & \multicolumn{3}{|c|}{ PPD } & \multicolumn{3}{|c|}{$\mathrm{CD}$} \\
\hline & beta & Std. Err. & $\mathbf{t}$ & beta & Std. Err. & t & beta & Std. Err. & $\mathbf{t}$ \\
\hline (Constant) & & 0.705 & 7.68 & & 0.029 & -0.30 & & 0.020 & -63.77 \\
\hline SPIRITI & -0.11 & 0.007 & -16.26 & -0.15 & 0.009 & -20.07 & 0.28 & 0.006 & 43.22 \\
\hline SPIRITII & 0.48 & 0.008 & 53.92 & 0.01 & 0.010 & 0.94 & -0.49 & 0.007 & -55.41 \\
\hline MINDI & -0.12 & 0.009 & -13.64 & 0.02 & 0.011 & 2.02 & 0.13 & 0.008 & 14.95 \\
\hline MINDII & 0.20 & 0.006 & 32.87 & 0.15 & 0.008 & 21.52 & 0.20 & 0.006 & 34.34 \\
\hline year & -0.07 & 0.000 & -12.42 & -0.12 & 0.000 & -18.72 & 0.36 & 0.000 & 66.02 \\
\hline gender & -0.12 & 0.009 & -23.73 & 0.06 & 0.011 & 10.15 & 0.02 & 0.008 & 3.89 \\
\hline \multicolumn{10}{|l|}{ Education level } \\
\hline Bachelor's & 0.14 & 0.012 & 19.21 & 0.05 & 0.896 & 6.52 & -0.04 & 0.011 & -5.45 \\
\hline Master's or higher & 0.18 & 0.013 & 24.26 & 0.02 & 0.896 & 2.39 & 0.07 & 0.012 & 9.12 \\
\hline \multicolumn{10}{|c|}{ Income level (INR/year) } \\
\hline $120.000-1,200.000$ & 0.14 & 0.011 & 24.42 & 0.15 & 0.896 & 21.59 & 0.09 & 0.009 & 14.88 \\
\hline$>1,200.000$ & 0.11 & 0.017 & 18.06 & 0.09 & 0.097 & 13.23 & 0.14 & 0.015 & 23.95 \\
\hline adjusted $R^{2}$ & \multicolumn{3}{|c|}{0.29} & \multicolumn{3}{|c|}{0.06} & \multicolumn{3}{|c|}{0.30} \\
\hline
\end{tabular}


Table 3.7: Regression results for various food choice and dietary behavior factors in dependence to trait spirituality and trait mindfulness factors (SPIRIT I, SPIRIT II, MIND I, and MIND II) and four socio-demographic traits (age, gender, education, and income) of USAmerican consumers. Level of significance at ${ }^{*} p<0.05$, ${ }^{* *} p<0.01$, and ${ }^{* * *} p<0.001$.

USA $(n=530)$

\begin{tabular}{|c|c|c|c|c|c|c|c|c|c|c|c|c|}
\hline \multirow[t]{2}{*}{ Trait } & \multicolumn{3}{|c|}{ IFC } & \multicolumn{3}{|c|}{ APD } & \multicolumn{3}{|c|}{ DA } & \multicolumn{3}{|c|}{ PEB } \\
\hline & beta & $\begin{array}{l}\text { Std. } \\
\text { Err. }\end{array}$ & $\mathbf{t}$ & beta & $\begin{array}{l}\text { Std. } \\
\text { Err. }\end{array}$ & $\mathbf{t}$ & beta & $\begin{array}{l}\text { Std. } \\
\text { Err. }\end{array}$ & $\mathbf{t}$ & beta & $\begin{array}{l}\text { Std. } \\
\text { Err. }\end{array}$ & $\mathbf{t}$ \\
\hline (Constant) & & 0.021 & 31.58 & & 0.018 & -3.96 & & 0.016 & -3.40 & & 0.020 & $\begin{array}{c}- \\
66.84\end{array}$ \\
\hline SPIRITI & 0.14 & 0.005 & 25.13 & 0.01 & 0.004 & -0.97 & $\overline{0} \cdot \overline{15}$ & 0.004 & 30.11 & 0.10 & 0.005 & 17.41 \\
\hline SPIRITII & 0.45 & 0.008 & 65.64 & 0.14 & 0.007 & 18.32 & 0.35 & 0.006 & 54.81 & $\overline{0 .}$ & 0.007 & -1.23 \\
\hline MINDI & 0.02 & 0.007 & 2.98 & 0.01 & 0.006 & -0.88 & 0.36 & 0.005 & 60.09 & 0.33 & 0.007 & 49.05 \\
\hline MINDII & 0.01 & 0.007 & 2.07 & 0.13 & 0.006 & 21.35 & 0.01 & 0.005 & -3.57 & 0.15 & 0.006 & 25.95 \\
\hline year & 0.23 & 0.000 & 42.10 & 0.06 & 0.000 & 8.21 & 0.01 & 0.000 & 1.62 & 0.35 & 0.000 & 60.48 \\
\hline gender & 0.10 & 0.010 & 19.61 & 0.24 & 0.009 & 39.66 & 0.10 & 0.008 & 19.70 & 0.08 & 0.009 & 16.50 \\
\hline \multicolumn{13}{|l|}{ Education level } \\
\hline College or associate's & 0.04 & 0.012 & -5.91 & 0.08 & 0.100 & 12.18 & 0.08 & 0.009 & 13.41 & 0.00 & 0.011 & 0.30 \\
\hline Bachelor's or higher & 0.05 & 0.013 & -7.49 & 0.15 & 0.011 & 20.45 & 0.10 & 0.010 & 16.52 & 0.12 & 0.012 & 18.37 \\
\hline \multicolumn{12}{|l|}{ Income level } & \\
\hline $35.000-100.000$ & 0.06 & 0.012 & 8.76 & 0.26 & 0.011 & 35.41 & 0.02 & 0.009 & 2.92 & 0.05 & 0.012 & 7.10 \\
\hline$>100.000$ & 0.15 & 0.014 & 21.65 & 0.17 & 0.012 & 21.96 & 0.17 & 0.011 & 25.63 & $\begin{array}{c}- \\
0.02 \\
\end{array}$ & 0.013 & -2.93 \\
\hline adjusted $R^{2}$ & & 0.31 & & & 0.13 & & & 0.40 & & & 0.27 & \\
\hline
\end{tabular}

USA $(n=530)$

\begin{tabular}{|c|c|c|c|c|c|c|c|c|c|}
\hline \multirow[t]{2}{*}{ Trait } & \multicolumn{3}{|c|}{ FV } & \multicolumn{3}{|c|}{ PPD } & \multicolumn{3}{|c|}{$\mathrm{CD}$} \\
\hline & beta & Std. Err. & $t$ & beta & Std. Err. & $t$ & beta & Std. Err. & $t$ \\
\hline (Constant) & & 0.019 & -13.65 & & 0.022 & -14.63 & & 0.024 & 0.26 \\
\hline SPIRITI & 0.02 & 0.005 & 3.84 & -0.03 & 0.005 & -4.99 & 0.01 & 0.006 & 1.41 \\
\hline SPIRITII & 0.34 & 0.008 & 44.73 & 0.45 & 0.008 & 60.36 & -0.38 & 0.009 & -50.45 \\
\hline MINDI & 0.02 & 0.006 & 2.99 & -0.14 & 0.007 & -19.41 & 0.16 & 0.008 & 22.71 \\
\hline MINDII & 0.08 & 0.006 & 12.72 & 0.03 & 0.006 & 5.66 & 0.19 & 0.007 & 31.61 \\
\hline year & -0.05 & 0.000 & -7.64 & 0.12 & 0.000 & 20.86 & 0.02 & 0.000 & 3.45 \\
\hline gender & -0.07 & 0.009 & -11.53 & -0.03 & 0.010 & -5.20 & 0.13 & 0.011 & 22.21 \\
\hline \multicolumn{10}{|l|}{ Education level } \\
\hline College or associate's & 0.04 & 0.011 & 5.96 & -0.09 & 0.012 & -14.22 & 0.04 & 0.013 & 6.21 \\
\hline Bachelor's or higher & 0.13 & 0.011 & 19.72 & -0.01 & 0.013 & -1.69 & -0.02 & 0.014 & -2.93 \\
\hline \multicolumn{10}{|l|}{ Income level(USD/vear) } \\
\hline $35.000-100.000$ & 0.16 & 0.011 & 21.99 & 0.08 & 0.013 & 11.83 & 0.07 & 0.014 & 9.54 \\
\hline$>100.000$ & 0.03 & 0.013 & 4.65 & 0.30 & 0.015 & 40.30 & -0.15 & 0.016 & -19.80 \\
\hline adjusted $R^{2}$ & \multicolumn{3}{|c|}{0.20} & \multicolumn{3}{|c|}{0.19} & \multicolumn{3}{|c|}{0.16} \\
\hline
\end{tabular}




\title{
Chapter 4 Towards a new Approach of addressing mindfulness in Sustainable Food Consumption Behaviors
}

\author{
With DR. ANTJE RISIUS
}

\begin{abstract}
In sustainable consumer sciences, the existing accounts conceptualizing and assessing mindfulness fail to resolve the contradiction between theoretical implications and behavioral outcome. While theories suggest that mindful consumers behave in more sustainable ways, studies that evaluate these relationships show mixed results. Therefore, a clear consensus of the effect of mindfulness on sustainable consumerism is still missing. The presented article addresses the question of how to optimize currently employed mindfulness concepts and scales to make research outcomes more meaningful and replicable. Drawing on additional features of traditional Indian Buddhism, this article introduces the term "right" mindfulness, which extends the current understanding of Western mindfulness by means of aspects of wisdom-based ethical and moral frameworks. Based on this more comprehensive understanding of mindfulness, its potential and applicability as a sustainability agent for food choice and diet is theorized. This paper also suggests potential ways to improve the development of mindfulness scaling or rating measures, especially with regard to self-report questionnaires. With this approach, the present article fosters greater interdisciplinary clarity concerning the scope, definition, and scale development of mindfulness, thereby laying the groundwork for further research into the relationship between mindfulness and sustainability.
\end{abstract}

\subsection{Introduction}

In sustainability sciences, the use of ecosystems and ecosystem services is framed around two distinct objectives of equity: intra-generational equality, and inter-generational equality (GLOTZBACH AND BAUMGÄRTNER 2009). Intragenerational equality is concerned with the well-being of all members of the present generation, while inter-generational equality considers the well-being of 
all members of later generations. In order to encourage societies on the path towards greater sustainability, strategies generally target the manipulation of collective and individual consumption behaviors (MULLER-EIE AND BJORNO 2016). For altering food consumption behaviors, those strategies can be grouped into three categories, namely restrictive actions, regulatory actions, and supportive actions (SPILLER et al., 2017). Restrictive actions enforce sustainable food consumption behavior by banning certain food products or by implementing standards for product manufacture and sourcing. Regulatory actions refer to the purposeful use of positive stimuli (by way of subsidies and incentives, for example) or negative stimuli (imposing taxes and fees) to guide consumption towards more sustainable behavior. Supportive actions are framed around the provision of information (measures such as labeling, education, and transparency) which aim at empowering consumers to make more informed decisions. However, these strategies, though of a certain scope and reach, are limited in how effectively they influence individual behavior since unsustainable dietary patterns are still on the rise (MONTEIRO et al. 2013, WHITNALL AND PITTS 2019).

For this reason, increasing attention has been paid to understanding the psychological mechanisms that motivate consumption behavior (see for example McKenzie-Mohr and Oskamp 1995, Pelletier et al. 2008, Verdugo 2012). Lately, the concept of mindfulness as a catalyst for promoting sustainability-relevant behaviors has received growing attention within the research community. The theoretical accounts linking mindfulness to consumption behavior emphasize its various positive effects on the human psyche including on decision-making processes. Based on that, sustainable food choice and dietary behavior is promoted by (1) the disruption of routines through observation, (2) bridging of the attitude-behavior gap through increased awareness, (3) increase of subjective well-being leading to temperance in consumption, and (4) increased incidence of pro-social behavior through the emergence of greater compassion (FISCHER et al. 2017). Because of these promising implications, mindfulness is regarded as a possible catalyst for inducing long-term behavior change that could lead to less environmental 
pollution, more balanced resource use, and healthier dietary behavior (FISCHER et al. 2017).

However, studies relating mindfulness to food consumption patterns (for instance Amel et al. 2009, Armstrong 2012, Geiger et al. 2019, Werner et al. 2020) or to the dynamics of behavioral change (see TAPPER et al. 2009, KEARNEY et al. 2012, RICHTER AND HUNECKE 2020) show ambiguous results. In these studies, mindfulness traits or interventions affect sustainability-relevant food consumption behaviors and dietary habits only partially or even negatively.

A possible reason for these inconsistencies may be the lack of agreement on how to conceptualize mindfulness in the modern research context. Mindfulness has become an umbrella term encompassing various definitions, explanations, and interpretations (BAER 2011, ChIESA 2013, Grossmann 2017). Another reason for the ambiguity of study findings may be found in the limited capacity of self-report questionnaires to accurately capture the complexity of mindfulness as this is closely interwoven with a range of behavioral, cognitive, moral, and social aspects (GROSSMAN AND VAN DAM 2011). Due to these shortcomings, claims for conceptual clarification and coherent scale development have already been advanced by a host of scientists (such as BLACK 2011, Grossman and van DAM 2011, SAUER et al. 2013, PURSER AND MILILLO 2015, FISCHER et al. 2017).

This article responds to these claims by providing a more holistic framework for mindfulness. The currently applied theoretical concepts for addressing mindfulness in sustainability sciences are clarified and enriched by using more traditional accounts of mindfulness in order to make research efforts in sustainable food consumption more accurate, replicable, and comparable. In this way, the authors attempt to spark more interest in a topic that still awaits deeper scientific consideration within the research context of sustainable consumption.

The article starts with an overview of mindfulness concepts and scales currently applied in studies focusing on food choice and dietary behavior change. Thereafter, drawing on aspects derived from traditional Indian Buddhism, additional aspects of the mindfulness concept are introduced to enrich the 
currently applied concept. Focusing on these additional aspects, mindfulness is defined as full awareness and conceptualized through four essential functions: (1) simple awareness, (2) protective awareness, (3) introspective awareness, and (4) deliberately forming conceptions. The last section of this article is concerned with integrating the introduced theoretical concept into actual research encounters. Readers are provided with a detailed description of important terminology, and the paper also provides suggestions for how to translate these additional aspects into self-report questionnaires, specifically as part of scientific approaches to sustainable consumption. This article thus hopes to foster greater interdisciplinary clarity on the scope, definition, and scale development of mindfulness and to provide a foundation for successfully integrating it into sustainability sciences.

\subsection{Current Mindfulness Approaches applied to addressing Food Choice and Dietary change Behavior of Consumers}

\subsubsection{What is Mindfulness? A Western Approach of an Eastern Concept}

Even though the Buddhist concept of mindfulness is around 2500 years old, its appearance in Western sciences is relatively recent, starting around the 1960s. Professor JON KABAT-ZINN played a crucial role in defining and interpreting the term as he was the first to introduce the idea of mindfulness into Western medical health sciences. Bringing mindfulness to a secular plane, he defined it as "paying attention in a particular way: on purpose, in the present moment, and non-judgmentally" (KABAT-ZINN 2014). The purposeful disassociation from Buddhism was based on the assumption that people who suffer and who could hence profit from mindfulness practices may be deterred from the Buddhist approach because they do not identify with its cultural and spiritual ideology (KABAT-ZINN 2000). Hence, popular therapeutic mindfulness approaches like the Mindfulness Based Stress Reduction (MBSR) method and, later, Mindfulness Based Cognitive Therapy (MBCT), have been developed as secular tools for mental health care.

Since these methods have proven effective in counteracting some mental health disorders and social issues (BARRETT et al. 2016), the concept of 
mindfulness has been receiving growing attention by scientists from other scientific disciplines like neuroscience and education. As a result of this growing interest in the phenomenon, the exploration of mindfulness within sustainable consumer sciences started to gain momentum two decades ago and has seen a significant increase in scientific articles over the last ten years (WAMSLER et al. 2018).

Even though this growing interest in mindfulness has added to significant theory development and understanding, it has also blurred definitions of the notion of mindfulness. More and more scientists and experts have added to or refined Kabat-ZINN's definition. For example, MarLATT AND KRISTELLER (2003) defined mindfulness as "bringing one's complete attention to the present experiences in a moment to moment basis". SEGAL and colleagues (2002) described it as "an opened attention that regards whatever enters as experience, while staying kindly curious to investigate whatever appears, without falling prey to automatic judgment or reactivity." BISHOP and colleagues (2004) understood mindfulness as "bridging non-elaborative awareness to current experience with an orientation of curiosity, experimental openness, and acceptance." Neurological scientists have referred to mindfulness as "the ability to filter and consciously select which intero- and exteroceptive information to attend to and construe meaning from. It comprises both eliminative and generative mechanisms" (DelLE FAVE et al. 2011). In sustainability sciences, the often cited review article on mindfulness and pro-environmental behavior by ERICSON and colleagues (2014) refers to mindfulness as present-moment awareness that includes everything that happens within the individual and in the surrounding environment.

In an attempt to summarize various Western definitions of mindfulness, BLACK (2011) identified four features common to these definitions. These are: (1) present-moment awareness, (2) present-centered intentionality, (3) experiencecentered cognitive attention, and (4) liberation of automatic and habitual response behavior. Present-moment awareness describes the mental state of actively attending a moment. Present-centered intentionality refers to the quality of present-moment awareness and is associated with openness, acceptance, 
and insight, while experience-centered cognitive attention focuses on the ability to consciously receive and respond to inner and outer stimuli. The liberation of automated and habitual response behavior can be understood as a natural byproduct of states of increased awareness.

\subsubsection{Western Mindfulness as a Catalyst for stimulating sustainability-relevant Food Consumption Behaviors}

The growing interest in mindfulness as a potential driver in navigating food choice and dietary behavior towards greater sustainability is based on the assumption that it is relatively easy to establish, maintain, and nurture. As a result, more and more researchers are exploring the possible effects of mindfulness on sustainable consumption behaviors. For example, the essay on mindfulness and consumption by ROSENBERG (2004) suggests that mindfulness stimulates traits of compassion, empathy, and care. As a result of this, mental states of non-reactivity, well-being, and happiness are promoted which are expected to increase pro-environmental and pro-social behavior. Similar implications of mindfulness on consumption behavior have been put forward by BALL and colleagues (2001), and ERICSON and colleagues (2014).

Increased states of mindfulness are considered to stimulate neurological reflexivity which leads to behavioral insight and, by this means, to changes in behavior (see figure 4.1). In particular, behavioral changes are promoted through strengthening (1) attention control, (2) emotional regulation, and (3) self-awareness (TANG et al. 2015). 


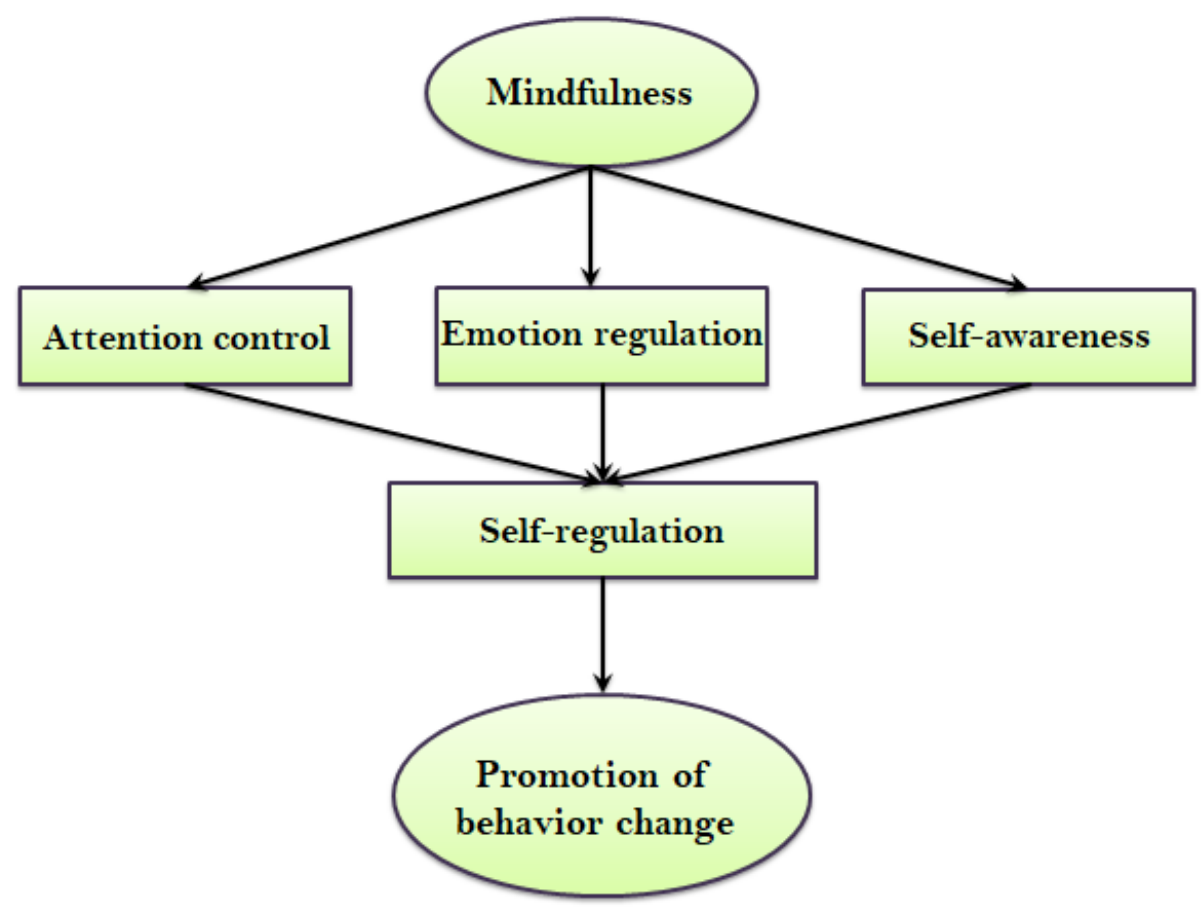

Figure 4.1: The integrative brain model for behavior change through mindfulness, taken and adapted from Tang and colleagues (2015).

Attention control affects the process of learning and remembering which influences an individual's ability to process information. Emotional regulation refers to the ability to process emotions including the ability to foster or neglect emotions, their duration, and how these are experienced and expressed. Selfawareness is the ability to perceive the self as an object of attention - that is, the ability to actively observe mental processes. These three qualities, then, enhance conscious and subconscious mental processes.

In line with these assumptions, the central hypothesis of this article is that mindfulness could be an important prerequisite for translating sustainability concepts into practical action. However, in order to examine whether mindfulness could be an important potential driver of sustainable development, it is crucial to be able to measure it accurately.

\subsubsection{Commonly used Mindfulness Scales for exploring Food Choice and Diet}

With mindfulness being investigated in various scientific fields, more and more scales and measures assessing mindfulness have been developed. In Western sciences mindfulness is measured as either a trait or a state. Trait mindfulness 
is argued to be dispositional whereas state mindfulness is associated with a state of being (LANGER 1989). However, this differentiation has been viewed critically by several scholars. For example, Grossman AND VAN DAM (2011) argue that most Buddhist traditions refer to mindfulness as a state that needs to be cultivated. By measuring mindfulness as a stable trait, as many Western scholars do, essential perspectives on mindfulness as a self-development process are left unconsidered (PURSER AND MILILlo 2015). To the best of the author's knowledge, studies in sustainable consumer sciences mainly use scales and measures to examine trait mindfulness. Both trait and state mindfulness are generally evaluated by means of psychometric scales (SAUER et al. 2013). The predominant mode of response is through self-report questionnaires with a Likert-type scale response, and the degree of mindfulness is estimated through additive weighting schemes.

In sustainability sciences, the three most frequently used scales are: (1) the Mindful Attention Awareness Scale (MAAS) (BROwN AND RYAN 2003; used for example by Brown and KaSSer 2005, PANno et al. 2018, Dhandra 2019), (2) the Five Facet Mindfulness Questionnaire (FFMQ) (BAER et al. 2008; used for instance by AMEL et al. 2009, BARBARO AND PICKETt 2016, HUNECKE AND RICHTER 2019), and (3) the Comprehensive Inventory of Mindfulness Experiences (CHIME) (BERGOMI et al. 2013; used by, among others, GEIGER et al. 2019, StANSzUs et al. 2019, WeRnER et al. 2020).

The MAAS is a 15-item scale designed to measure trait mindfulness through reverse scaling. The scale is based on the definition that mindfulness refers to "the state of being attentive to and aware of what is taking place in the present" (BROWN AND RYAN 2003). Accordingly, the two major components used to evaluate the degree of mindfulness are attention and awareness. The former refers to an individual's ability to focus on certain objects or stimuli, while the latter refers to a person's ability to actively perceive internal and external stimuli without putting a specific focus on them (BROWN AND RYAN 2003).

The FFMQ also measures trait mindfulness. Its development is based on the combination of various commonly used mindfulness questionnaires including the MAAS. BAER and colleagues (2006) adopt the more common definition of 
mindfulness as present-moment awareness and add the components of nonjudging and acceptance. The FFMQ consists of 39 statements that evaluate an individual's mindfulness based on five dimensions, namely: (i) observing, (ii) describing, (iii) acting with awareness, (iv) non-judging of inner experience, and (v) non-reactivity to inner experience. Observing refers to an individual's awareness of thought processes and emotions (an example statement would be "When I'm walking, I deliberately notice the sensations of my body moving"). Describing refers to one's ability to describe and identify thoughts and emotions ("I'm good at finding words to describe my feelings"). Acting with awareness is the capacity to stay present with regard to an object or mental state without getting distracted ("I do jobs or tasks automatically without being aware of what I'm doing"). Non-judgment of inner experience captures the capacity for nonsubjective observation of thoughts and emotions (like "I criticize myself for having irrational or inappropriate emotions"). The last dimension, non-reactivity to inner experience, refers to an individual's potential to stay calm when facing troubling inner states (for example, "I watch my feelings without getting lost in them").

The CHIME measure was developed by BERGOMI and colleagues (2013). Mindfulness is defined as a two-component construct as suggested by BISHOP and colleagues (2004). The first component is concerned with the selfregulation of attention in order to stay in the present moment, while the second component refers to how one ought to pay attention, which should be in a curious, open, and accepting way. Similar to the FFMQ, development of this measure has been based on a thorough analysis of existing mindfulness scales, including the MAAS and the FFMQ. The CHIME comprises eight dimensions: (i) awareness of internal experiences, (ii) awareness of external experiences, (iii) acting with awareness, (iv) accepting and non-judgmental attitude, (v) nonreactive decentering, (vi) openness to experiences, (vii) awareness of thought/relativity, and (viii) insightful understanding. The first two dimensions measure the ability to actively respond to one's emotions (inner awareness) and to external stimuli (outer awareness). Acting with awareness represents an individual's capacity to stay present within everyday activities. Acceptance and non-judgmental attitude refers to one's ability to remain objective in the 
presence of unsettling internal and external stimuli. The fifth dimension, nonreactive decentering, concerns the active detachment from unwholesome states of mind. Openness to experience represents an individual's capacity to attend to and be present with stimuli as they appear. Awareness of thought/relativity captures the observation of mental states and emotions, and the final dimension, insightful understanding, emphasizes the importance of experience to foster insight. Trait mindfulness is evaluated through a 37 -item scale.

Even though all scales measure the same idea, they differ in how they construct their scales (for example, forward vs. reverse scaling), terminology (referring to "awareness" rather than "attention", for instance) and dimensionality (using two vs. eight dimensions). Therefore, when employing existing rating systems like the MAAS, FFMQ, CHIME, or other scales, researchers are encouraged to thoroughly reflect on the nature and interpretation of constructs of mindfulness that arise from these scales. Otherwise, the significance of the findings as well as the comparison of results may be inappropriate or misleading. The development of a consistent construct for assessing mindfulness in sustainability sciences would therefore contribute to a clearer classification, interpretation, and delineation of empirical findings.

\subsubsection{Inconsistencies in the Mindfulness-Sustainability Relationship: between Theory and Empirical Research}

As mentioned above, theories of mindfulness in the context of sustainable consumption point to various positive effects on the human psyche, leading to a natural temperance in consumption (ROSENBERG 2004, FISCHER et al. 2017). However, empirical evidence about the relationship between mindfulness and sustainable consumption behavior is inconsistent. For example, AMEL and colleagues (2009) hypothesize that mindfulness reduces automatic behavioral responses by enhancing attentive decision-making about consumption. Their study linked the FFMQ subscales "acting with awareness" and "observing" with self-reported green (or ecologically attentive) consumption behavior. The study found a significant positive relationship between the ability to stay present with situations (acting with awareness) and green consumption choices. However, ambiguities where found between the ability to observe thoughts (observing) 
and green consumption behavior. In contrast to theoretical approaches that associate "observing" with the reduction of habitual response behavior (FISCHER et al. 2017), this study found only non-significant negative associations between these two variables.

Another study explored the effect of mindfulness interventions on reducing the attitude-behavior gap (GEIGER et al. 2019). University students and white collar employees attended an adapted mindfulness-based training for at least five out of eight weekly sessions. Mindfulness was assessed before and after the training sessions using the CHIME scale. Even though the training generally increased trait mindfulness as measured through the CHIME, it did not affect sustainability-related food consumption behavior nor associated sustainabilityrelevant attitudes.

Finally, WERNER and colleagues (2020) explored the relationship between trait mindfulness and parameters of sustainability-relevant food consumption. Trait mindfulness was assessed through online questionnaires using a shortened version of the CHIME. They found significant positive relationships between some of the mindfulness subscales and dietary awareness as expressed through dietary diversity, well-being, and awareness of dietary habits. However, in contrast to theories that considered mindfulness to enhance dietary sustainability (ROSENBERG 2004, FISCHER et al. 2017), the study results pointed to an increase in self-reported dietary intake of convenience foods and animalprotein based foods.

Empirical research into the relationship between mindfulness and behavior change dynamics is relatively limited, especially in the area of sustainable consumption. In two studies, BARBARO AND PICKETT (2016) examined the relationship between mindfulness, connectedness to nature, and self-reported pro-environmental behavior. The pro-environmental behaviors evaluated comprised waste behaviors, shopping choices, and transportation. Mindfulness was measured using the FFMQ. The studies found that mindfulness is positively associated with pro-environmental behavior. Furthermore, connectedness to nature can act as a significant mediator in this relationship. Based on these findings they concluded that mindfulness reduces mental barriers for behavioral 
change by connecting to the object of behavior. Similar implications of mindfulness on the mechanisms underlying behavioral change were emphasized by the authors of a previously mentioned study (AMEL et al. 2009). In another study, RICHTER AND HUNECKE (2020) used a stage model of behavioral change to investigate the relationship between mindfulness and organic food consumption in more detail. The model comprised ten stage variables, among which were social norms, attitudes, goal intentions, and perceived behavioral control. Similar to the studies conducted by AMEL and colleagues (2009) and BARBARO AND PICKETT (2016), the FFMQ was used to assess mindfulness. They found an indirect association between mindfulness, especially for the subscale "observing", and the goal of an intention to consume organic food. However, the effect of mindfulness as an explanatory variable within the model was marginal.

Comparably more research into the relationship between mindfulness and behavioral change dynamics stems from the healthcare sector, specifically with regard to dietary issues and eating disorders. In this regard, TAPPER and colleagues (2009) explored the effects of mindfulness-based trainings on weight-loss behavior in order to draw a clearer picture of those psychological characteristics, particularly experiential avoidance, linked to obesity. Measurements relating to emotional, external, and binge eating, physical activity, mental flexibility, avoidance of difficult psychological states, and dietary adherence were taken before, during, and after a six month mindfulness-based intervention. The results showed significant positive behavioral changes with regard to performing physical activities. However no significant effects on psychological flexibility, weight loss, and emotion-based or externally influenced eating behaviors were found. Similar hypotheses on the relationship between mindfulness-based interventions and dietary change behavior have been examined by KEARNEY and colleagues (2012). However, in contrast to TAPPER and colleagues (2009), this study focused on the deeper exploration of emotional eating and uncontrolled eating behaviors in a predominantly male sample. The study used traditional MBSR training, lasting for six months, to stimulate the cultivation of greater mindfulness. Measurements were taken once before the commencement of the MBSR training, after the training ended, and 
four months after that. Scaling of trait mindfulness was based on the FFMQ (BAER et al. 2008). The study found that higher levels of mindfulness generally decrease emotion-based and uncontrolled eating behaviors. However, mere participation in the MBSR intervention did not show any effect on the investigated eating behaviors. Furthermore, no significant changes in the consumption of sugars, fats, vegetables, and fruits were recorded. In another study, JORDAN and colleagues (2014) investigated the link between trait mindfulness and calorie intake. The complete research format comprised four individual studies evaluating food choice and dietary intake. Mindfulness was assessed using the MAAS scale (BROWN AND RYAN 2003). While the first two studies focused on general effects of trait mindfulness on dietary behavior, the other two explored the effect of mindfulness on dietary change through an induced mindfulness-based manipulation of respondents before confronting them with a task to rate different snack foods. Overall, increases in trait mindfulness reduced uncontrolled eating behaviors. Furthermore, the induced mindfulness-based manipulation led to a decreased intake of calories and shifted food choices towards healthier alternatives (like fruit instead of sweets). Even though implied by theory, this effect was not related to changes in the strength of self-control and ego-depletion.

In sum, these studies reveal that empirical evidence on the potential of mindfulness as a sustainability agent is inconsistent with theoretical implications regarding food consumption behavior and behavioral change. The next chapter will therefore provide a framework for a more comprehensive understanding of mindfulness and suggestions for how to apply it more effectively in sustainable consumer sciences.

\subsection{Towards a Holistic understanding of Mindfulness: reintroducing Eastern Concepts}

\subsubsection{The Eastern Concept of Mindfulness}

Whereas KABAT-ZINN'S (2014) definition of mindfulness builds on a secular notion of mindfulness as a cognitive mode of functioning, the traditional Eastern understanding of mindfulness largely derives from the discourses of the Buddha and is closely tied to Eastern spiritual concepts such as rebirth. Here, the 
cultivation of mind extends to aspects of morality, insight, and emotional stability (KUAN 2012).

Several descriptions of mindfulness are available in Buddhist literature. Within the Abhidhamma Pitaka (around the 3rd century BCE), an assembly of Buddhist texts that provide a structured compilation of Buddhist thought, sati (mindfulness) was referred to as "recollection, recalling, remembrance, a keeping or bearing in mind, absence of floating, and absence of forgetfulness". Recurring elements from the Buddhist translation of sati also refer to the state of present-moment awareness and non-judgmental acceptance (BODHI, 2013, DREYFus 2011). RhYS DAVIDS AND RHYS DAVIDS (1966-1971) extended the scope of sati by adding that its most important quality is the realization of the temporariness of everything that exists, including physical and mental states. A more detailed explanation of the features of mindfulness was given in the Visuddhimagga (THOMAs 1929). Here, mindfulness was described as "not wobbling, its function is non-confusion, it is manifested as guarding, or as facing the object. Its proximate cause is firm perception, or its proximate cause is the foundation of mindfulness of the body, and so on. Because it is firmly supported, it should be viewed as a pillar or as a gate-keeper because it guards the eye-door, and so on." This guarding or gate-keeping quality of mindfulness points to an ethical component present in Eastern conceptions of mindfulness. Here, mindfulness involves an active component that questions common structures and circumstances that counteract the greater good of all, including oneself. Accordingly, PAYUTTO (1988) pointed out that all meanings of sati should remind a person of their duties, that one should be constantly present and aware of the conditions under which one comes into contact and act appropriately.

The most complete description of mindfulness practices from the Buddhist perspective is given in the Satipaţ̧̦hāna Sutta. This states that mindfulness, when cultivated properly, enables an individual to use their full mental capacity. In Buddhist philosophy, mindfulness leads to mental liberation as it frees the mind of mental conditioning, automatism, and subjective judgment. The person is enabled to take the right decisions for the greater good of all and to adjust 
individual actions accordingly. Hence, mindfulness promotes independent thinking and a flexible mind. KUAN (2008) draws a clearer picture of the importance of mindfulness in cultivating wholesome mental states by explaining the four essential functions of mindfulness in the Buddhist literature. These are: (1) simple awareness, (2) protective awareness, (3) introspective awareness, and (4) deliberately forming conceptions (see figure 4.2).

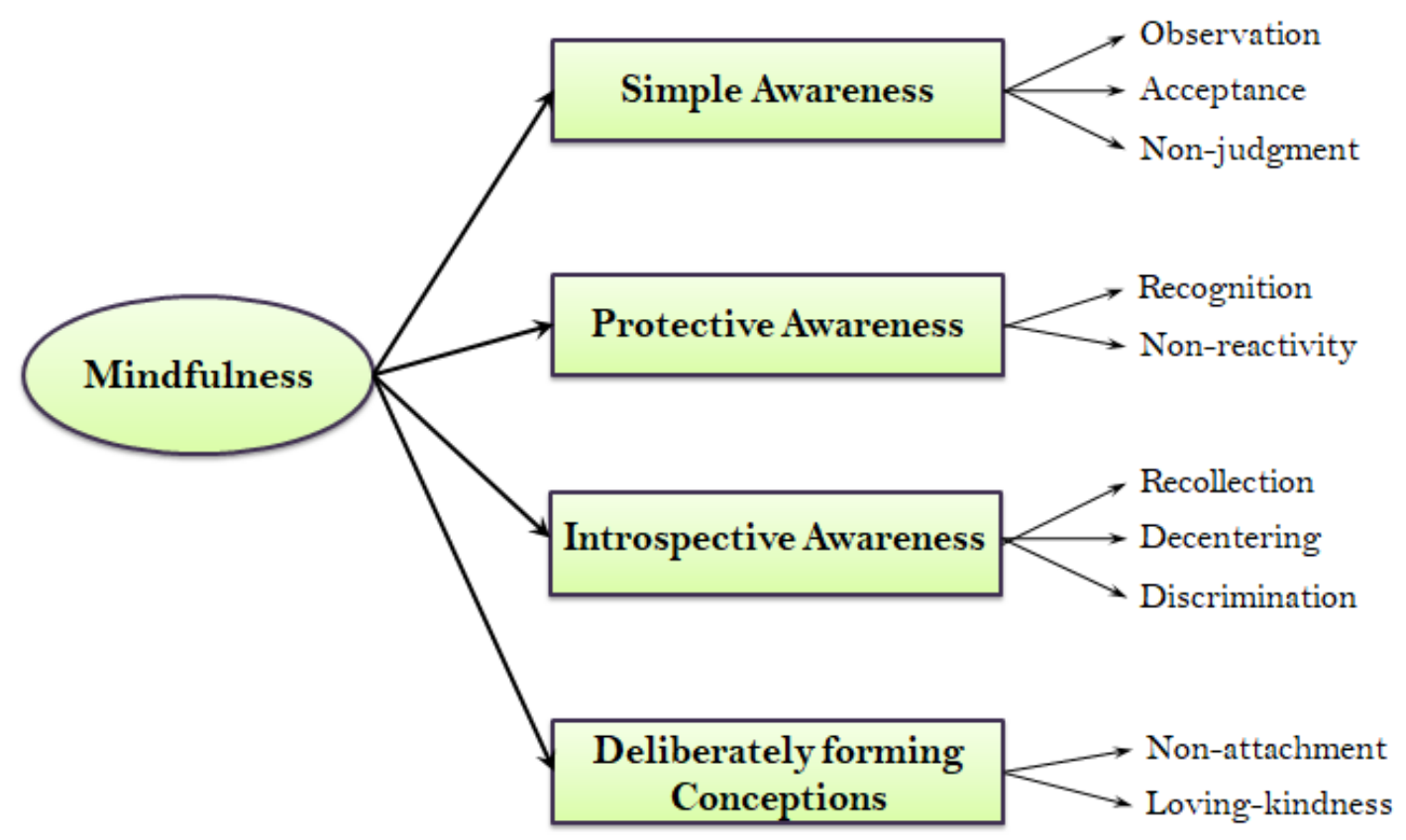

Figure 4.2: The four essential functions of mindfulness according to Kuan 2008 (modified).

The first function, simple awareness, refers to the bare registering of an object that is presented to the senses; its constituents are observation, acceptance, and non-judgment. Most research frameworks within sustainability sciences focus on studying mindfulness with respect to this function only. As a result, the concept of mindfulness is often limited to the qualities of bare attention and nonjudgment without considering the deeper psychological mechanisms that mindfulness can stimulate and affect. The next function of mindfulness is protective awareness. It comprises the act of noticing how the mind reacts to an object. In opposition to simple awareness where the (mental) object is perceived, in protective awareness, the cognitive process itself is observed and recognized. Furthermore, a decisive component, based on non-reactivity, is added. Because of this, in Buddhism, protective awareness is considered a gate-keeper that only allows wholesome mental states to enter the mind. 
Through this restraining of the senses, wholesome mental states (like kindness, compassion, sympathetic joy, and equanimity) are consciously invited whereas unwholesome mental states (such as carelessness, sluggishness, ignorance, and restlessness) are repelled. Introspective awareness operates in cases where unwholesome mental states are readily present within the mind. In this function, mindfulness serves as a reminder of various mental states, their proper discrimination, and either nurturance (in the case of wholesome mental states) or dissolution (in the case of unwholesome mental states). Through this, the skillful evaluation of emerging mental states is encouraged, and this reduces habitual responses to stimuli. The fourth function of mindfulness, deliberately forming conceptions, concerns faith, memory, and remembrance. However, it does not refer to the recall of past personal experiences but to the process of transforming apperception through intentional conceptualization. This function predominantly aims at overcoming overwhelming emotions, especially fear, through non-attachment, reminding the individual of wholesome attributes and traits (like wisdom, faith, and morality) but is also used to cultivate loving-kindness, a specific kind of feeling that is closely associated with mindfulness. Along with compassion, altruistic joy, and equanimity, loving kindness is one of the four sublime emotional states in Buddhism (SAXENA 2019), and can be characterized as the authentic wish for everyone to be happy and well (VELEZ DE CEA 2019).

It is important to note that these four functions are closely interrelated. For example, the second and third, protective and introspective awareness, generally operate together and are motivated by the fourth function, that of deliberately forming conceptions (KUAN 2008). Skillful mindfulness is, therefore, the result of mastering all four functions.

\subsubsection{Why it is Important to cultivate Mindfulness skillfully}

In Buddhism, mindfulness is further refined by differentiating between right, or skillful, mindfulness, and wrong, or unskillful mindfulness (GETHIN 2001). The former means going along with, appropriate, thoroughly, or properly; the latter means wrong but also coarse or unrefined. In opposition to unskillful mindfulness which is depicted as being forgetful, easily distracted and with a 
self-centered attentiveness, right mindfulness involves the attributes of kindness, non-attachment, and the tendency to act selflessly rather than selfishly (GETHIN 2015).

KUAN (2008) clarified this terminology by explaining that mindfulness itself is a given unskillful quality of the mind. Right, or skillful, mindfulness, however, is a component of the Noble Eight-factored Path (ariyo atthangiko maggo), a set of Buddhist practices that are meant to free the mind of distress. The path elements are: (1) right view, (2) right thought, (3) right speech, (4) right action, (5) right livelihood, (6) right striving, (7) right mindfulness, and (8) right concentration (see figure 4.3). It is noteworthy that "right", in the context of the Noble Eight-factored Path, refers to personal understanding and not to perfection. This means that, even though all relevant aspects are considered as closely as possible, it remains clear that there is a natural limit to human accountability. For example, the adjective in "right view" refers to the "most comprehensive view that can be adopted within the limitations of human knowledge and understanding" (KALUPAHANA 1995).

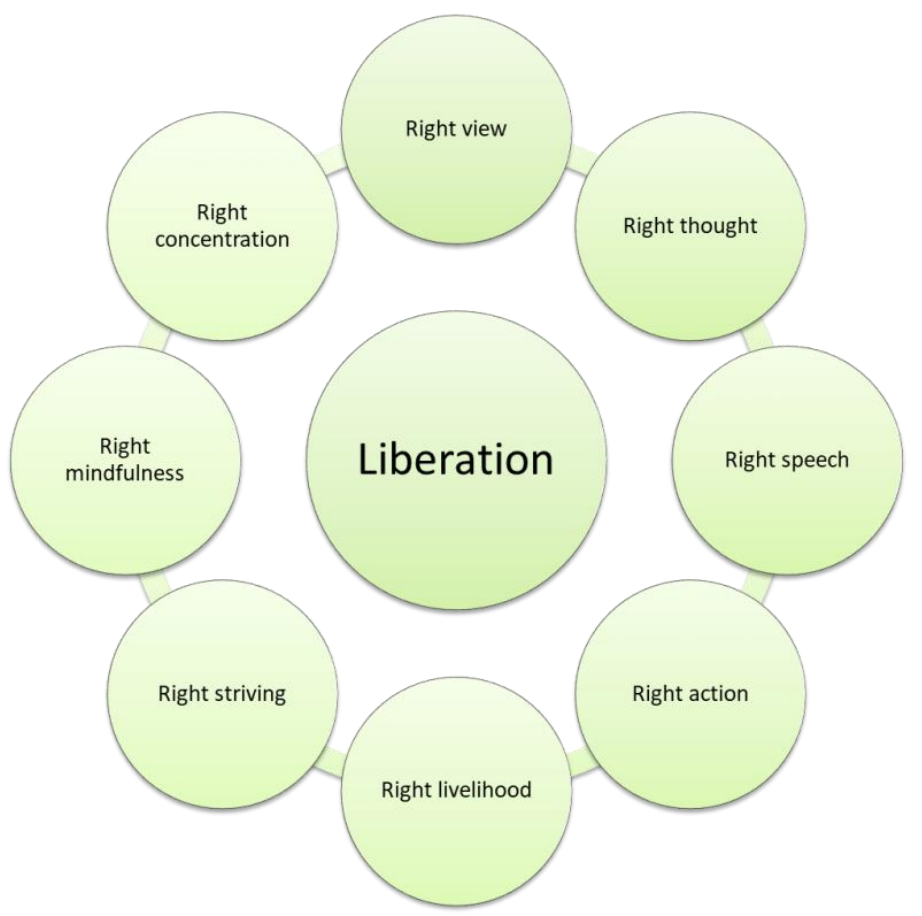

Figure 4.3: The elements of the Buddhist Noble Eight-factored Path to liberation.

The first two factors are considered as the foundation of wisdom and are based on ethics and virtue. Right view (1) comprises knowledge with regard to human 
suffering, its origins, cessation and dissolution. Right thought (2) refers to the intentionality of mind which should be free of desire, friendly, and compassionate. Factors three to five represent the result of mental will and hence represent moral conduct. In this respect, right speech (3) is the active abstaining from lying, scheming, or hurtful speech, and malicious gossip. Right action (4) refers to the ability to act selflessly towards other beings and includes abstention from stealing, killing, and sexual misconduct. Right livelihood (5) describes the ability to make a living without compromising personal values and ethics. The remaining three factors within the Eight-factored Path are considered to be mental disciplines. Right striving (6) refers to the effort necessary to cultivate skillful mental states like generosity or wisdom and to dissolve unskillful mental states like greed or ignorance. Right mindfulness (7) comprises the ability to be fully present within situations, thoughts, and feelings and plays an important role in objectivity and skillful judgment. Even though the path elements are interconnected and build upon each other (PURSER AND MILILLO 2015), the last factor, right concentration (8), is dependent on mastering all the previous seven factors. It is described as the ability to remain focused on a single object leading to freedom from delusion and separateness (Kuan 2008).

Only right mindfulness can guide apperception (saññā) (HAMILTON 1996). A crucial component of sañña is that it is entirely based on subjective judgment if it is not mastered through the cultivation of right mindfulness (THERA 2005). Eventually, this subjective perception will be memorized and can therefore influence future experience (GETHIN 2001, THERA 2005). In this regard, the importance of right mindfulness, therefore, stems from its ability to support the mind in perceiving objects as they truly are without being attached to an agenda. This objective view then allows the individual to organize occurring internal and external sensations based on their relative value and obviates abusive acts based on selfishness. Hence, the common belief of Western scholars that mindfulness is inherently non-judgmental is not fully supported by traditional Buddhist conceptualizations of mindfulness. Here, non-judgment is important to the first function of mindfulness only, which is simple awareness (KUAN 2008, see figure 4.2). As depicted in figure 4.4, if based on skillful ethical 
and moral foundations (like right view and right thought), mindfulness practice will lead to insight as things can be objectively recognized, connected, and evaluated. By means of deeper insight, all previous path factors are strengthened and these will in turn support the emergence of right mindfulness deep within the human psyche. Eventually, this will lead to right concentration and liberation of suffering. Therefore, within the larger context of personal growth, right mindfulness may be understood as the bridge between intellectual insight and experience. The foundational elements, right mindfulness, and insight strengthen and support each other until liberation from (mental) suffering is achieved.

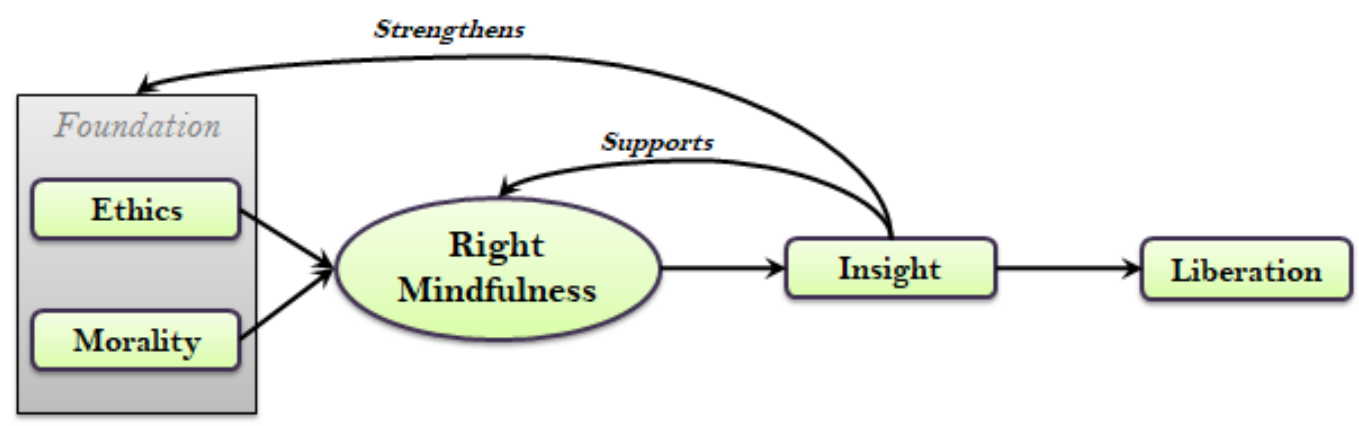

Figure 4.4: The position of right mindfulness in the process of mental liberation.

Right mindfulness should not be restricted solely to meditation practice. Indeed, practicing mindfulness through different means is important for intellectual understanding, but applying it in everyday life is equally important for the integration of mindfulness (GETHIN 2015). KUAN (2008) differentiates between internal and external objects of mindfulness. The former refers to mental and physical sensations observed during meditation, while the latter describes the observation of extrinsic objects, such as the actions and thoughts of others, and needs to be cultivated alongside meditative practice. Hence, meditation leads to an understanding of right mindfulness while experience acts as a necessary catalyst for integrating right mindfulness. Accordingly, a person who has cultivated right mindfulness, not just intellectually but also through experience, will not be reactive and merely unconsciously responsive to stimuli but will instead act in a centered, observant, and aware manner. This state will increase inner harmony and add meaning to everything an individual does because it is done with the unity of body and mind meeting authentically at the same space 
which can only be (in) the present moment. This connection between right mindfulness and insight may be crucial not just in bridging the intentionbehavior gap but also in cultivating new kinds of consumption pattern that are based on self-responsibility.

\subsubsection{Eastern Mindfulness and its Connection to Buddhist Spirituality}

As mentioned above, Western scholars intentionally left spiritual or religious ideologies out of the mindfulness concept in the hope that it would gain greater acceptance within Western society (PURSER 2019). However, a closer investigation of the primary relationship between mindfulness and spirituality may prove valuable in order to understand, differentiate, and cultivate mindfulness correctly.

In his review, KALE (2004) defined spirituality as "the engagement to explore and deeply and meaningfully connect one's inner self - to the known world and beyond". Accordingly it consists of four aspects: (1) a sense of the inner self, (2) a sense of meaning, (3) a sense of interconnectedness, and (4) a sense of the beyond. The sense of the inner self is mostly associated with metaphysical ideas of the soul and of higher consciousness. Worldly matters, like human experience and life purpose, constitute the sense of meaning. The sense of interconnectedness relates to the inherent bond between human and matter, and a sense of the beyond refers to the existence of the supernatural realm. In opposition to religion which is associated with institutionalization, hierarchy, conformity, and social affiliation, spirituality is considered to be more personal, flexible, and informal (HARVEY 2016). In Buddhism, spirituality is not related to a deity but to the complete understanding of natural laws. GETHIN (2001) describes Buddhist faith as "a distinct and skilful positive emotional response of confidence". Therefore in contrast to Western concepts, the Buddhist idea of faith is not cognitive but purely affective. This means that faith is based on experiential confidence or universal trust and not on beliefs in higher powers or propositions (GETHIN 2001).

Furthermore, the concept of self differs fundamentally between West and East. Even though this has been little discussed in the literature, the concept of self is 
a crucial component in understanding the origins and necessity of mindfulness and mindfulness practices. The Western concept of self-understanding is largely influenced by Sigmund Freud. He categorized the mind into three divisions: the id, ego, and superego (MCLEOD 2016). The id is driven by basic biological needs and instincts like hunger or sex. The ego is a result of environmental and psychosocial factors like the desire for prestige or acknowledgement. In opposition to the id, the ego is not driven by the satisfying of pleasure but by reasoning. The superego represents the instance of conscience as it mediates between id and ego, and is hence deemed to be the foundation of morality and ethics. Based on these understandings, psychoanalysis aims at supporting people in adapting to given social norms and structures like earning a living or building relationships (LIANG 2009). The Buddhist understanding of the self, however, is different. The ego is considered an illusion projected by the mind due to the fear of nonexistence (Liang 2009). As a result, mental habits are perceived as faulty and unwholesome in nature (GETHIN 2015). This is the reason why traditional Buddhist perspectives suggest freeing the mind from these habits and thought patterns, even when these are reinforced by pre-existing social and cultural factors. The Buddhist conception of self is therefore an expression of non-self or selflessness based on complete detachment from any agenda (personal and social) but focused on the highest good of all.

The association between mindfulness and spirituality stems from the understanding that mindfulness, if cultivated correctly, enables individuals to gain a constant awareness of consciousness through emotional and mental transformation (KUAN 2012). However, the application of mindfulness as a spiritual tool is utterly dependent on the individual's intentions.

\subsubsection{The Role of Ethics and Morality in the Cultivation of Right Mindfulness}

Ethics and morality are closely connected but are not the same. Morality comprises a set of widely agreed upon and accepted standards that are regarded as "good" and "bad" between groups of people that make up societies. Ethics, however, refers to the process of reflecting upon these moral standards (GAmmeL, n.d.). Compared to Western philosophical systems, Buddhism does 
not feature one specific work on ethics and morality (AITKEN 2016). This does not mean that ethical precepts are not to be found but, rather, that these are scattered throughout Buddhist writings. Perhaps because of this, there is confusion among Western scholars with regard to determining Buddhist approaches to ethics and morality. However, AITKEN (2016) suggests that Buddhist ethics follows ideas that are not anchored in any Western ethical framework. Therefore, classifying them as consequentialism or virtue ethics, as is usually done by some authors in the West, may lead to de-contextualization and misinterpretation, because this neglects the Buddhist ethical account of mental transformation. He summarizes Buddhist thought on ethics and morality as follows:

"A common theme that runs through Buddhist ethical writings is the notion that the way we experience the world is the foundation for moral concerns. On this account, the moral problem is the fact that we are ordinarily confused about the nature of the world and our place in it, which leads to a life characterized by the experience of suffering and vice. The moral solution, then, is the removal of this confusion through the development of a metaphysically accurate understanding of the world and our place in it, which transforms the way we experience the world in such a way that frees us from suffering, promotes virtues, and brings us to a morally mature state."

The process of becoming morally mature is reflected in the Buddhist Noble Eight-factored Path (see figure 4.3). Buddhist ethical accounts are driven by insight but rooted in right view and right thought. Moral conduct comprises the path factors right speech, right action, and right livelihood. GETHIN (2015) summarizes the important implications of moral conduct for the cultivation of the remaining path elements by pointing out that they are the "essential manifestations of mental will", and have two qualities. The first quality continuously cultivates skillful virtues by practicing right speech, right action, and right livelihood, and the second abandons unskillful qualities by refraining from wrong speech, wrong action, and wrong livelihood. The potential of 
mindfulness then is rooted in an increased awareness of personal ethics and moral conduct.

Ethics and morality have been widely neglected in Western conceptualizations of mindfulness. This means that the foundation upon which Western mindfulness is cultivated and developed is inconsistent and unknown. Buddhist scholars stress that these inadequacies in addressing ethical and moral standards within the Western mindfulness concept will eventually lead to a misappropriation and thus weakening of the potential of mindfulness (GREENBERG AND MitRA 2015). In fact, concerns with regard to the neglect of ethical and moral dimensions when employing mindfulness constructs have also been expressed by KABAT-ZINN (WILLIAMS AND KABAT-ZINN 2011).

\subsection{Towards an integrated Understanding and Assessment of Mindfulness in Sustainable Food Consumption}

This article argues that a concept originally designed for clinical patients with health issues may be limited in its applicability to the greater social context of sustainable development. This assumption is reinforced by empirical studies suggesting that health issues influence consumption behaviors like diet, as the motivational dynamic guiding fundamental consumption decisions changes (see MASKARINEC et al. 2001). In this regard, it seems as if the Western mindfulness concept exaggerates the effect of mere meditation practice on the mind and mental habits, and consequently on decision-making. Traditional accounts of right mindfulness, however, are integrated into a broader ethical and moral framework, encouraging objective (self-) reflection, selfless action, and independent thinking and insight (GETHIN 2001). Hence, they reach far beyond meditative practice. Thoroughly integrated theoretical approaches for exploring mindfulness in sustainable consumption are therefore vital for understanding the application, scope, and relevance of this relatively abstract concept for sustainable behavioral change in food choice and diet.

The following chapter starts by presenting theoretical implications and important definitions to promote an integrated conceptualization and clear understanding of mindfulness and the relevant terminology. Suggestions for how to translate 
these conceptual implications into a coherent mindfulness scale are presented thereafter.

\subsubsection{Conceptualization and Integration of Mindfulness into the Sustainability Framework}

This article proposes to define mindfulness as full awareness. This comprises simple, protective, and introspective awareness, as well as the ability to deliberately form conceptions (see figure 4.2). Furthermore, the integration of ethical, moral, and wisdom-based frameworks is highly recommended in order to establish a thorough understanding of its effect on decision-making and behavior. Based on the suggestions given by TANG and colleagues (2015), figure 4.5 depicts the position and relevance of right mindfulness within the process of cultivating new means of behavior.

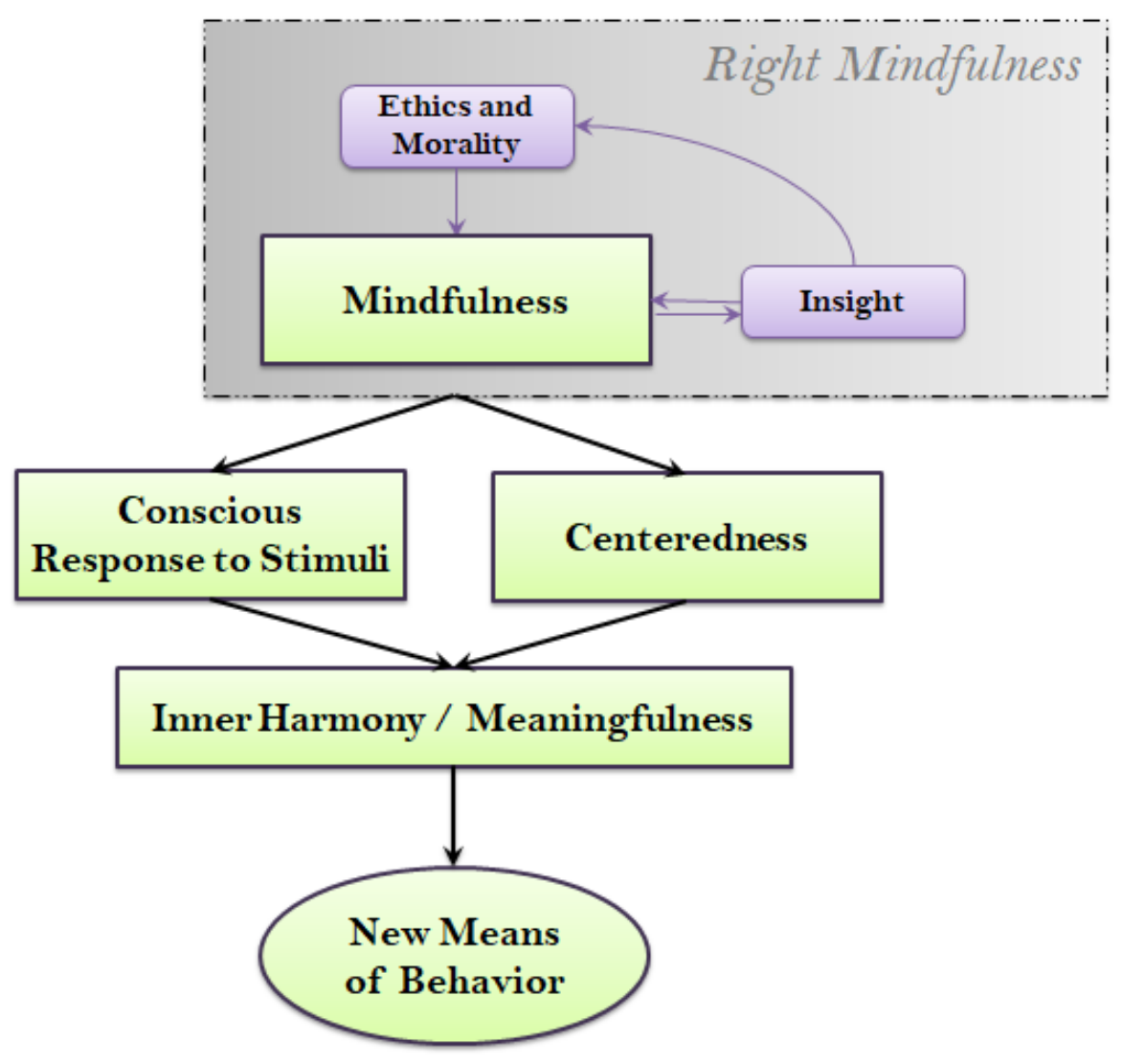

Figure 4.5: The position of right mindfulness within the process of behavior formation.

As the above shows, the relationship between ethics, morality, mindfulness, and insight is not one-directional but mutually dependent. Hence, the efficacy of the whole process is limited by its weakest constituent. Cultivating mindfulness in 
order to counteract cognitive problems or emotional disturbance can thus only work alongside the dimensions of ethics, morality, and insight (KUAN 2008). Starting from these foundations, right mindfulness enables the individual to attend to experience in the immediate present (Bodhi 1998) which reduces unconscious responses to mental stimuli. Moreover, the alignment with personal virtues is promoted by the recognition of cause-effect relationships that underlie phenomena. This leads to greater emotional balance and, through this, an increase in centeredness. Eventually, greater centeredness and more consciousness in response translate into states of inner harmony and meaningfulness arising as the larger context of the true self is understood distinct from proscribed labels given by, for instance, family members, societies, or religious affiliations. Hence, the inherent value and structure of experience as well as its ethical implications are understood (AITKEN 2016), leading to new patterns of consumption behavior by focusing awareness on the mental activities that influence experience. Through this, mindfulness practice becomes more meaningful as a catalyst for changes in consumption behavior as it generates experience-based insights about mental states and their impermanence, as well as understanding of the effects of wholesome and unwholesome mental activities (AITKEN 2016).

However, the distinction between good and bad must be based on an integrated perspective of the genuine nature of circumstances. Therefore, thorough ethical and moral foundations are considered essential for cultivating right mindfulness. It includes the questioning of and, if necessary, the breaking with commonly followed social ideologies, conventions, and moral conduct (QUEEN AND KING 1996, HАM 2000, ОMVEDT 2003). In the context of consumption, sustainability is often restricted to a particular institutional and social environment (SHWOM AND LORENZEN 2012). Hence, food consumption dynamics partly depend upon given supply structures. If sustainable food options are nonexistent or difficult to access, consumption behavior may not reflect consumers' true choices. In this regard, the ability to question given supply structures and actively seek better options is necessary for pushing sustainable development forward. Accordingly, VERMEIR AND VERBEKE (2006) regard the ability to reflect on current social structures as one of the most important drivers for long-term sustainability in 
consumption. However, this requires the proper observation, integration, and judgment of given structures as well as a clear idea of personal ethics and morality. In this respect, right mindfulness may be a key ingredient for initiating behavior change as it emphasizes the moral viewpoints of mental states.

\subsubsection{Important Terms and Definitions}

Reviewing the current literature available from both research topics, a dissonance appears in the interpretation of some definitions. Therefore, in order to strengthen the overall understanding of terminology relevant to the concept of mindfulness, this section defines and clarifies important terms. The nine terms discussed within this sub-chapter include: (1) awareness, (2) attention, (3) insight, (4) acceptance, (5) observation, (6) decentering, (7) non-reactivity, (8) non-attachment, and (9), non-judgment.

One of the most common buzzwords to describe mindfulness is awareness (1). Accordingly, various facets of awareness are characterized within current mindfulness accounts, such as present-moment awareness, acting with awareness, or awareness of internal and external experiences. The CAMBRIDGE DICTIONARY (n.d.) defines awareness as: "knowledge that something exists, or understanding of a situation or subject at the present time based on information or experience". However, since awareness is a central component of Western mindfulness concepts and measures, different scholars have defined the term more specifically, resulting in various slightly differently worded definitions. This article will focus on the definitions most common in sustainability sciences. BROWN AND RYAN (2003) understand awareness as "the background 'radar' of consciousness, continually monitoring the inner and outer environment." Baer and colleagues (2006) describe acting with awareness as the "ability to stay present with an object or mental state". BERGOMI and colleagues (2013) distinguish between three key fields of awareness which are related to the ability to attend to feelings, thoughts, and situations. Even though awareness is generally considered to have an experiential quality, it is not explicitly included in the definitions given by BROWN AND RYAN (2003), BAER and colleagues (2006), or BERGOMI and colleagues (2013). In Eastern schools of thought this kind of awareness is mostly described as "simple awareness" (see figure 4.2), 
and is understood as the beginning of the awareness process and one that comprises four further aspects. In this respect, GETHIN (2015) points out that awareness is a process that promotes an objective perception of feelings, thoughts, behaviors, and situations by counteracting unconscious habits and preconceived ideas. Therefore, the term "full awareness" goes beyond bare attention but includes the intention to counter unwholesome states of mind (KUAN 2008).

Awareness is promoted through meditation. It should be noted that silent-sitting meditation is not the only form of meditation, even though it is commonly associated within Western approaches. An excursus on different meditation techniques, however, would go beyond the scope of this paper. Interested readers are recommended to read MATKO AND SEDLmEIER (2019) to gain an overview of meditation practices. We therefore stick to explaining the central goal of every meditation - learning to observe. A detailed definition of the term "observation" will be given below.

Like awareness, attention (2) seems to play an important role in the Western mindfulness concept. KABAT-ZINN $(2000,2014)$ refers to mindfulness as focused conscious attention or as awareness that stems from paying attention. Attention means "thought, notice, or interest" (CAMBRIDGE DICTIONARY n.d.). Brown and Ryan (2003) describe it as the ability to focus the mind on specific objects. Within Buddhism, attention plays a crucial role, too, as the ultimate constituents of reality (dhammas) are considered to be generated from the arising of attention (KUAN 2008). Therefore, attention, in Buddhist notions of mindfulness, is not associated with the specific way someone pays attention but rather with where and upon what an individual focuses attention (GETHIN 2015). Accordingly, GETHIN (2015) defines attention as the mental process that turns the mind "toward the object of awareness in each moment of consciousness".

Attention and awareness are easy to confuse. To avoid misuse of terminology, one may differentiate between awareness and attention by the mental space it occupies. Attention takes up space - that is, a person can only pay attention to either one thing or another. Awareness, however, creates space, which implies that a person can be aware of more than one individual thing at a time. 
Another important term that needs further clarification is insight (3). The Cambridge Dictionary (n.d.) defines it as "a clear, deep, and sometimes sudden understanding of a complicated problem or situation." In the CHIME questionnaire, insight is described as an ability that fosters understanding of thoughts and feelings in a certain way that allows comprehension of the "inner workings of the mind". JOHNSON and colleagues (2017), who designed a CHIME questionnaire for young adolescents, interpreted insight as an understanding of the suffering engendered by subjectivity. In Buddhism, insight is defined as the ability to recognize and reorganize personal experience mediated through the senses (KUAN 2008). The four functions of mindfulness (see figure 4.2) are considered the pathway to insight. In turn, insight supports the further cultivation of right mindfulness. Two types of insight are distinguished: ordinary insight, and penetrative insight (GETHIN 2001). The former refers to a state of insight where an individual accumulates information by attending to only one (mental) object at a time. Here, insight is the result of a process. The latter type, on the other hand, is a sudden transcendence leading to immediate insight.

Acceptance (4) is commonly defined as the "ability to fully experience situations and circumstances without defense" (HAYES 1994). Through the inclusion of feelings and thoughts into experience without pre-judging, an individual will inevitably comprehend the full range and scope of emotions and thoughts present within them. Acceptance of unconscious tendencies is especially important as this will lead to balanced mental states if it is applied to the body, feelings, consciousness, and mental objects (KUAN 2008).

Observation (5) is crucial to mindfulness. BERGOMl et al. (2013) refer to observation as the objective experience of thoughts and feelings as they come and go without falling into self-identification. Likewise, the FFMQ describes observation as the attending to internal and external experience (BAER et al. 2006). The Buddhist scholar GETHIN (2015) refers to this term as the mere observation of the (mental) "objects that are presented to the senses". In both interpretations, observation is completely detached from a personal agenda but merely observes (mental) objects for what they truly are. Observation is 
disrupted either through identification with, or in response to, the observed (mental) object.

In Western concepts of mindfulness, decentering (6) is understood as an active detachment from unwholesome or troubling states of mind (BERGOMI et al. 2013). In Buddhism, decentering is especially necessary for achieving detachment from egocentric thinking and self-identification. In this process, the cause and effect relationship of thoughts, feelings, actions, and circumstances can be seen clearly (LIANG 2009). Traditionally, decentering is mastered when the truths of suffering (i), the source of suffering (ii), the end of suffering (iii), and the way to the termination of suffering (iv) are thoroughly understood and integrated (LIANG 2009). Therefore, decentering is closely related to the phenomenon of selflessness.

In Western mindfulness approaches, decentering is often connected to nonreactivity (7), which refers to the ability to not become involved with mental objects, like thoughts and feelings, as they occur. In Buddhism, however, nonreactivity is connected to insight (KUAN 2008). It comprises the ability to counteract automated response to mental stimuli, based on the understanding of what causes them (WHITMARSH 2013).

The aspect of non-attachment (8) reflects the so-called Buddhist middle path. It is based on the assumption that attachment adversely affects emotional wellbeing as it causes desire and excessive rumination (COFFEY et al. 2010). As a result, attachment leads to suffering and unhappiness. To counteract the clinging of mind, non-attachment is cultivated through observation and acceptance. Hence, non-attachment is not indifference or aversion as is sometimes misinterpreted by researchers. It does not imply an absence of feelings and emotions but rather concerns how these are acknowledged. One should not cling to any particular emotional state but instead remain in an observing position. Through this, subjective perception is bridged into objectivity, thus allowing for wholesome judgment.

For the mindfulness scales most commonly used in sustainability sciences, elements of non-attachment are often labeled in terms of non-reactivity. 
However, this might be misleading. Though both concepts reflect the Buddhist middle path on how to deal with emotions, non-attachment emphasizes the quality of "letting go", whereas non-reactivity refers to the quality of "nonavoidance".

Being non-judgmental (9) is another important foundation of Western mindfulness concepts. Non-judgment is defined as the ability to accept feelings, thoughts, sensations, and perceptions objectively and without over-identification (BERGOMl et al. 2013). From this viewpoint, non-judgment is considered to be crucial for acceptance, self-reflection, disruption of routines, and affective reactivity (DREYFUS 2011). In Buddhism, non-judgment describes the process of "noting things as they occur" (GRIFFITHS 1981, cited in KUAN 2008). It is therefore associated with simple awareness but not with protective and introspective awareness or with deliberately formed conceptions. In simple awareness, judgment leads to an unskillful discrimination of mental objects. Through discrimination, situations and circumstances are evaluated by matching them to personal views and values. Unskillful discrimination occurs in cases where the response to a mismatch between personal virtues and stimulus leads to unwholesome emotions like anger or fear. The quality of nonjudgment refers to the ability to remain aligned with personal virtues without being triggered if situations and circumstances do not align with them. It is concerned with the ability to authentically acknowledge thoughts and feelings rather than staying neutral or taking a non-evaluative stance towards them. This explanation shows that in Buddhism non-judgment does not refer to any particular act of judgment but is concerned rather with not condemning thoughts, feelings, and circumstances. The organization of these within personal values, ethics, and moral conduct schemes, however, is central to the process of cultivating right mindfulness. Therefore, non-judgment is not about controlling thoughts and emotions, as often suggested in mindfulness scales, but rather about the ability to listen to mental activities and how they inspire the individual in exploring the presence of judgment within the mind. 


\subsubsection{Implications for developing a Scale to assess Mindfulness}

Currently, the way mindfulness scales are applied within sustainability-relevant food choice and dietary parameters has proven problematic with regard to comparability and, consequently, the significance of results. For example, while the MAAS exclusively focuses on the aspects of attention and awareness, the FFMQ and CHIME suggest a multifaceted nature of mindfulness. Hence, although these scales stem from the same concept, comparing scale results may prove difficult as different conceptual interpretations, delineations, and terminologies come into play. Furthermore, the three commonly applied mindfulness scales (MAAS, FFMQ, and CHIME), in their current versions, fail to assess key characteristics of right mindfulness. For this reason, these scales may not be the best choice for assessing mindfulness in relation to consumption patterns. Designing enhanced constructs is therefore highly recommended.

As depicted in figure 4.5, this article proposes constructing a measure that can assess mindfulness by including its aspects of moral maturity. Initial suggestions for drawing up such a scale have been provided by PURSER AND MILILLO (2015). They outlined the Triadic Mindfulness Model, an ethically informed framework for assessing corporate mindfulness. Based on the Noble Eight-factored Path, the three model dimensions are: right view, right striving, and right mindfulness. Right view represents ethical values, right striving is a complementary interconnected motivational force strengthening the remaining dimensions, and the assessment of right mindfulness is predominantly based on the capacity to recollect, remembering personal virtue, and motivational effort. In this model, right view is crucial as it nurtures the foundations of what is right or wrong.

In line with the findings of PURSER AND MILILLO (2015), this article recognizes the scientific value deriving from assessing the elements that make up the Noble Eight-factored Path. However, to assess right mindfulness in food choice and diet, a slightly different theoretical model is now proposed (see figure 4.6). 


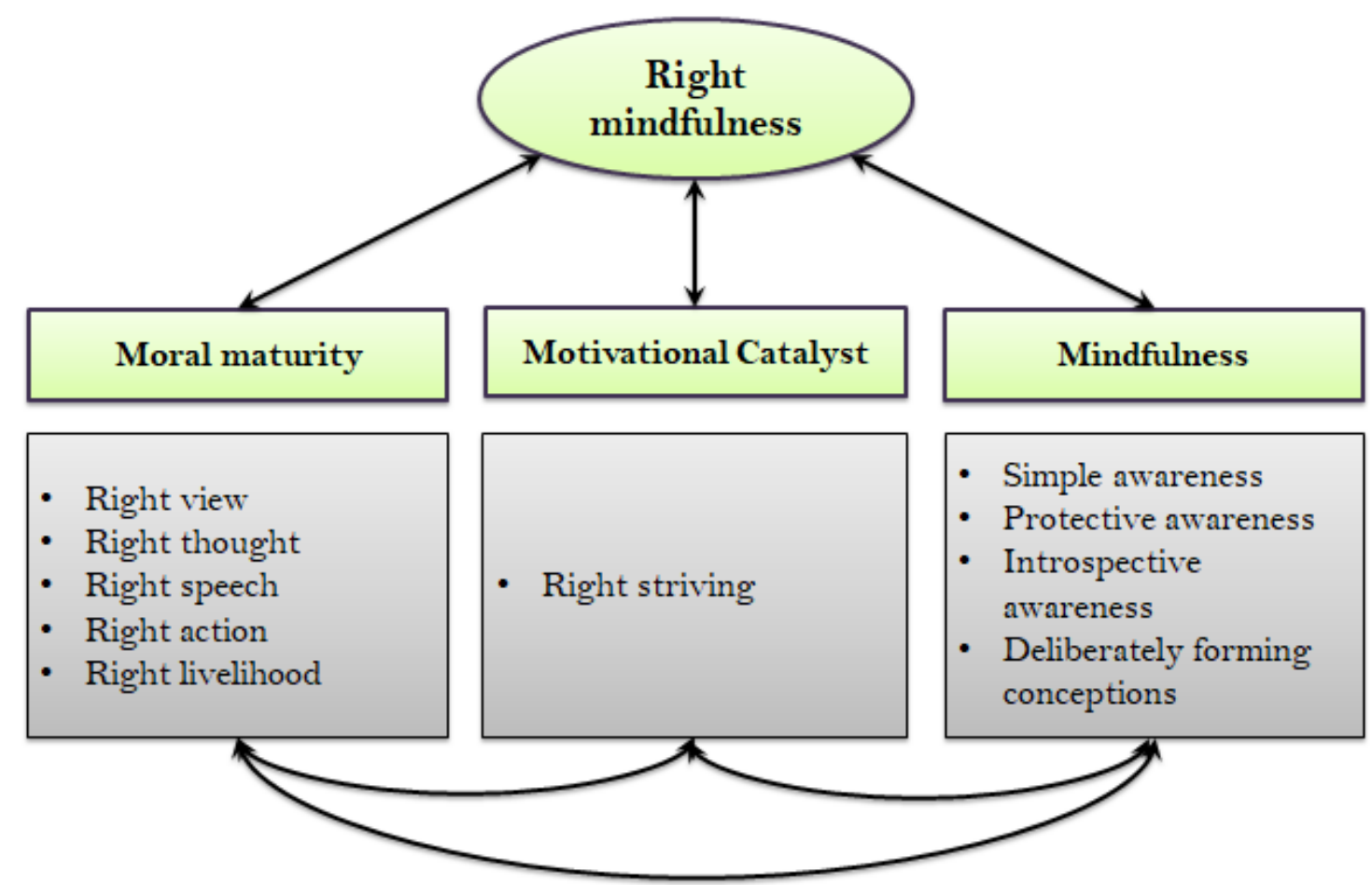

Figure 4.6: A proposed theoretical model for measuring right mindfulness in sustainability sciences.

The proposed theoretical model comprises three interdependent dimensions: (1) moral maturity, (2) motivational catalyst, and (3) mindfulness. Moral maturity reflects the alignment with sustainability-relevant ethical and moral viewpoints regarding consumption (concerning social justice and the ethical treatment of animals, for example) but also wisdom-based independent thinking. The relevance of right striving as a motivational driving force for mental development is adopted from PuRSER AND MILILLO (2015). The last dimension, mindfulness, consists of the four functions of mindfulness (see figure 4.2 for an overview of the components shaping each function). Scale items for assessing the four functions of mindfulness should be designed in the light of the explanations and definitions provided within this article (see chapters 4.2 and 4.3). Furthermore, in contrast to recent studies, we recommend basing the calculation of the degree of right mindfulness on multiplied or weighted additive indices rather than on additive scaling schemes in order to account for the interdependence of the three dimensions.

As far as the author is aware, all currently available scales assess mindfulness through textual statements only. However, as mindfulness is closely connected 
with cognition and emotion, this article recommends integrating scale items that stimulate respondents on various cognitive and emotional planes. For example, visual material can generate emotional responses and self-identification more strongly than text material which respondents absorb mainly on an intellectual level (JOFFE 2008). The rating of visual materials is therefore valuable for estimating emotional regulation and can indirectly provide information on a respondent's degree of protective and introspective awareness. Another option, though one that necessitates a great deal of data analysis, is the use of openresponse items (such as asking respondents to indicate buzzwords) as these stimulate different intellectual processes like goal- or essence-based thinking (DASHEN AND FRICKER 2001). Information on respondents' modes of thinking could contribute extensively to the collection of accurate data about the dimensions of moral maturity and drivers of motivation. This is also true for items that require respondents to indicate their preferred ways of acting in certain hypothetical situations. In this regard, scholars are advised to use different cognitive stimuli when presenting hypothetical situations, with such stimuli including images, video, audio, and text material.

\subsection{Final Remarks}

In conclusion, the strength of mindfulness measures in sustainability sciences depends on a thorough understanding of the position and role of mindfulness within the process of behavior change. While the current field of Western mindfulness science may have contributed greatly to the implementation of therapeutic measures, inconsistencies in studies linking mindfulness to sustainability behaviors call for scientists in this area to rethink and adjust existing measures.

In this regard, the presented article contributes to a more refined understanding of contemporary discourse around mindfulness. Ideas about how to approach the relationship between mindfulness and sustainable food consumption include hitherto largely neglected aspects of experience based on moral maturity. These extensions to the currently used mindfulness framework draw on traditional literature relating to Buddhist notions of mindfulness. Similar suggestions for reintroducing ethics and morality into mindfulness have also 
been proposed by Grabovac and colleagues (2011), PurSer and Milillo (2015), and MONTEIRO and colleagues (2015).

GRABOVAC and colleagues (2011) argue that ethical frameworks decrease doubt, worry, and guilt, leading to a reduction in mental proliferation. Mindfulness can then be understood as a tool for aligning personal ethics with behavior. PURSER AND MILILLO (2015) point out that current mindfulness frameworks in use are incomplete and can even prove counterproductive if they fail to take into account ethics and morality, as they reduce the individual's ability to critically engage, discern, and reflect on situations and circumstances. In their article on traditional and contemporary mindfulness, MONTEIRO and colleagues (2015) raise similar concerns with regard to the limited scope of contemporary mindfulness concepts. They see major shortcomings especially with respect to the sustainability of treatment outcomes and claim that, without ethical guidelines, a merely symptomatic and short-lived relief from distress is provided. Both the articles PURSER AND MiLILLO (2015) and of MONTEIRO and colleagues (2015) stress the use of right mindfulness in Western mindfulness sciences.

The conceptual considerations provided in this article aim at supporting researchers in understanding the role of right mindfulness within the greater framework of sustainable consumption. The design of enhanced scales, including aspects of moral maturity, motivational force, and mindfulness is recommended for ensuring a more accurate assessment of right mindfulness. Further research should therefore focus on operationalizing a rating scale that includes and fully integrates these aspects. With this approach, researchers would be encouraged to practice right mindfulness by questioning the commonly used formats of traditional mindfulness scales. An adapted framework for measuring mindfulness, then, would add significant value to the examination of mindfulness within sustainability-relevant food choice and dietary behavior. 


\section{Appendices}

The organization of the appendices follows the structure of the dissertation. Accordingly, appendix A contains the additional materials relevant for essay one, appendix $B$ contains the additional materials relevant for essay two, and appendix $\mathrm{C}$ contains additional material for essay three.

\section{Appendix A}

The study for essay one was carried out in Germany. Therefore, the original material is in German language. The following account is a translation of the original material. It contains the following materials: (A.1) Interview guidelines, (A.2) Task 1: Motivations, (A.3) Task 2: Mental Models, and (A.4) Questionnaire.

\section{A.1 Interview Guidelines}

The materials used for the interviews comprised: recorder, chronometer, data privacy instruction sheet (according to article 13; Private Data Protection Law), Interview Guideline, Information sheet TEMS and VIVO, Sticker TEMS and VIVO, socio-demographic questionnaire, plain sheets and colored pens, pencils, receipt book, and remuneration (20 Euro). 


\section{A.1.1 Call for Participants - original German version}

Juli 2019

\section{Interviewpartner gesucht}

pas Department für Agrarökonomie und Rurale Entwicklung der Georg-August Universität Göttingen forscht, um neue strukturen für nachhaltige Ernährungsweisen zu entwickeln. Um ein besseres verständnis zu den intrinsischen Treibern alternativer Ernährungsstile aufzubauen suchen wir Menschen ab einem Alter von 18 Jahren die bereits längerfristig einen der folgenden Ernährungsstile verfolgen:

$\begin{array}{ll}\text { - Makrobiotische Diät } & \text { - LOGI Methode } \\ \text { - Mazdaznan Ernährung } & \text { - Zonen-Diät } \\ \text { - Waerland Diät } & \text { - Paleo-/Steinzeit Diät } \\ \text { - Schnitzer Normalkost } & \text { - Fit for Life } \\ \text { - Ayurvedische Diät } & \text { - Evers-Diät } \\ \text { - Diät in der traditionellen Chinesischen Medizin } & \text { - Hay'sche Trennkost } \\ \text { - Anthroposophische Diät } & \text { - Evers Diät } \\ \text { - Schnitzer Intensivkost } & \text { - Vitalstoffreiche Vollwertkost } \\ \text { - Rohkost } & \text { - Sonstigen alternativen Ernährungsstilen }\end{array}$

Im Rahmen von Tiefeninterviews werden neben ideologischen Gesichtspunkten auch Aspekte der Ernährungsumstellung besprochen. Der zeitliche Aufwand beträgt max. 2 Stunden. Eine finanzielle vergütung von 20,- $\mathbf{\epsilon}$ wird geleistet. Interviews können in den Städten Göttingen, Kassel, Hannover, Stuttgart und Berlin wahrgenommen werden.

Bitte melden Sie sich bei Interesse bei aspasia.werner@uni-goettingen.de

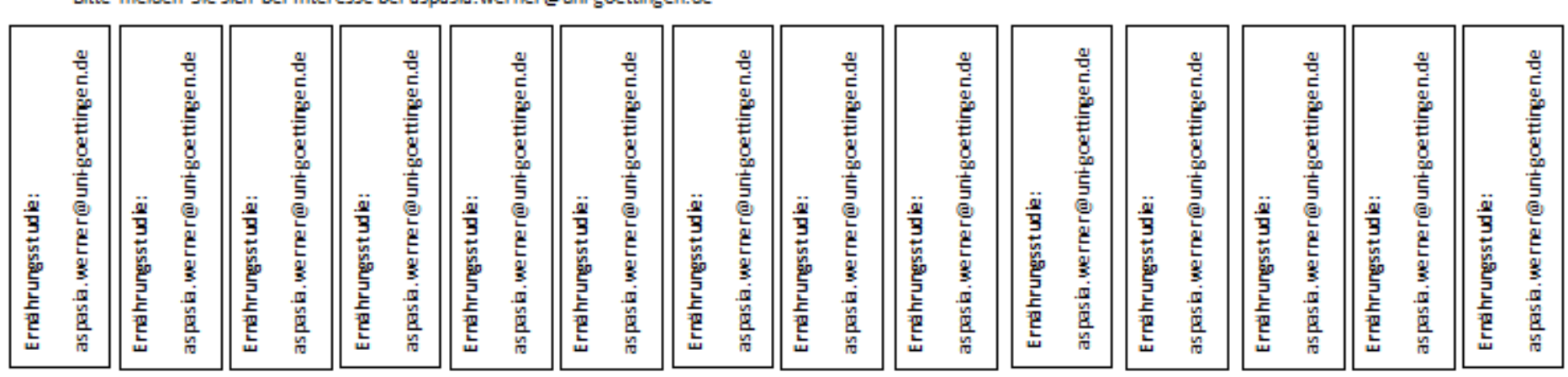




\section{A.1.2 General Structure}

\section{Basic Structure of the Interview}

\begin{tabular}{|c|c|c|c|}
\hline Module & Sequence & Content & $\begin{array}{l}\text { Time } \\
\text { [min] }\end{array}$ \\
\hline Arrival & Reception & Offering of beverage, seating, description to washrooms, etc. & 5 \\
\hline Data Security & Interview & $\begin{array}{l}\text { Before we start I would like to ask you to agree to the privacy policy by signing this document. This is } \\
\text { necessary to allow me to evaluate your personal data. I would like to inform you that I am recording our } \\
\text { conversation. I do this so that I can concentrate fully on our conversation and still not forget any details } \\
\text { later during the evaluation. I hope this is ok for you. Your data will be anonymised. Furthermore, the } \\
\text { recordings will be deleted after transcription of the interview has been finalized. }\end{array}$ & $2-3$ \\
\hline Icebreaker & Interview & Question 1 & $2-3$ \\
\hline Motivation & Interview & Questions 2-5 & $25-30$ \\
\hline Motivation & Interview & Task 1 & 15 \\
\hline Mental Model & Interview & Questions 6-7 & \\
\hline Obstacles & Interview & Question 8 & $5-10$ \\
\hline Mental Model & Interview & Task 2 & $20-25$ \\
\hline Mental Model & Interview & Questions 9-10 & \\
\hline Strategy & Interview & Question 11 & 5 \\
\hline Closing & Interview & $\begin{array}{l}\text { Thanks for your time. Finally, I would like to ask you to fill out this short questionnaire to collect your } \\
\text { socio-demographic data and some details about your diet. }\end{array}$ & 6 \\
\hline Farewell & & $\begin{array}{l}\text { Again, I would like to thank you very much for your participation. As agreed, I have remuneration for the } \\
\text { time and information you shared with me. }\end{array}$ & 3 \\
\hline
\end{tabular}




\section{A.1.3 Question Guideline}

Questions Guideline: Part I

\begin{tabular}{|c|c|c|c|c|}
\hline $\mathrm{Nr}$ & Module & Main question & Sub question & Aim \\
\hline 1 & - & What did you eat today? & & Icebreaker \\
\hline 2 & Motivation & How would you describe your dietary lifestyle? & & $\begin{array}{l}\text { Orientation dietary lifestyle } \\
\text { / Basic information }\end{array}$ \\
\hline \multirow[t]{2}{*}{3} & Motivation & How many years have you been dieting this way? & & $\begin{array}{l}\text { Orientation dietary lifestyle } \\
\text { / Basic information }\end{array}$ \\
\hline & & & How did you eat before that? & \\
\hline \multirow[t]{4}{*}{4} & Motivation & What was the decisive point for the change in diet? & & Trigger / turning point \\
\hline & & & $\begin{array}{l}\text { Can you describe the process in more } \\
\text { detail? }\end{array}$ & Trigger / turning point \\
\hline & & & $\begin{array}{l}\text { What was of central importance in the } \\
\text { process? }\end{array}$ & Trigger / turning point \\
\hline & & & $\begin{array}{l}\text { What exactly did inspire you to } \\
\text { change? }\end{array}$ & Trigger / turning point \\
\hline \multirow[t]{5}{*}{5} & Motivation & $\begin{array}{l}\text { How long did it take from the idea to the implementation of } \\
\text { the new diet? }\end{array}$ & & Transition period \\
\hline & & & $\begin{array}{l}\text { Which aspects were a particular } \\
\text { challenge for you and why? }\end{array}$ & $\begin{array}{l}\text { Transition period / } \\
\text { obstacles }\end{array}$ \\
\hline & & & $\begin{array}{l}\text { Looking back, what was the biggest } \\
\text { hurdle for you during the transition? }\end{array}$ & $\begin{array}{l}\text { Transition period / } \\
\text { obstacles }\end{array}$ \\
\hline & & & $\begin{array}{l}\text { What kind of support would you have } \\
\text { wished for? (In terms of challenges) }\end{array}$ & Obstacles \\
\hline & & & $\begin{array}{l}\text { What supported you in staying on } \\
\text { track? }\end{array}$ & $\begin{array}{l}\text { Transition period / } \\
\text { obstacles }\end{array}$ \\
\hline
\end{tabular}




\section{Questions Guideline: Part II}

\begin{tabular}{|c|c|c|c|c|}
\hline $\mathrm{Nr}$ & Module & Main question & Sub question & Aim \\
\hline 6 & $\begin{array}{l}\text { Mental } \\
\text { Model }\end{array}$ & $\begin{array}{l}\text { You mentioned } X \text { as one of your main motivations. Can you } \\
\text { describe the meaning of that in more detail? }\end{array}$ & & Mental structrues \\
\hline 7 & & $\begin{array}{l}\mathrm{Y} \text { is rather irrelevant for your dietary change. So it had no } \\
\text { effect on you or do you mean that it demotivated you? }\end{array}$ & & Mental structures \\
\hline \multirow[t]{3}{*}{8} & Obstacles & $\begin{array}{l}\text { When do you find it difficult to stick to your current eating } \\
\text { style? }\end{array}$ & & Strategy \\
\hline & & & $\begin{array}{l}\text { What then gives you the strength to still } \\
\text { stick to your dietary philosophy? }\end{array}$ & Strategy \\
\hline & & & $\begin{array}{l}\text { Why don't you just eat what everyone } \\
\text { else is eating? }\end{array}$ & Strategy \\
\hline 9 & $\begin{array}{l}\text { Mental } \\
\text { Model }\end{array}$ & $\begin{array}{l}\text { You identify strongly with } \mathrm{X} \text {, what exactly do you associate } \\
\text { with it? }\end{array}$ & & Mental structures \\
\hline 10 & & $\begin{array}{l}\text { The least } Y \text { has applied to you why do you feel } \\
\text { unresponsive to it? }\end{array}$ & & Mental structures \\
\hline 11 & Strategy & $\begin{array}{l}\text { Finally, if you could give a piece of advice or a wish to } \\
\text { someone who wants to change their diet, what would it be? }\end{array}$ & & Strategy \\
\hline
\end{tabular}




\section{A.1.4 Task Guideline}

\section{Task Guideline}

Task description

Guiding Question

I would like to ask you to structure a mind map using these materials provided. These are basic motivations of food choice. Can you arrange them according to their importance for

1 Motivation your dietary change? You can find corresponding explanations of the individual motivations on the information sheet or you can ask me. If you want to add motivations you can use the

What is the motivation behind empty stickers.

Could you please assign the given statements to your personality? For this I provided a rough scale for your orientation. Please classify the statements according to their

truthfulness. The upper part of the scale corresponds to a characteristic that describes or

2 Mental Model defines you very well. The lower part of the scale is a characteristic or belief that does not

describe or define you at all. If you don't want to assign a statement, just stick it into the area at the bottom left of the poster. Please feel free to ask me for corresponding explanations of

Are there connections between specific mental models and the practice of alternative lifestyles? the individual statements. 


\section{A.2 Task 1: Motivations}

\section{A.2.1 Motivation Stickers and Information Sheet}

In total, 15 motivations were provided. These were: (1) liking, (2) habits, (3) need and hunger, (4) health, (5) convenience, (6) pleasure, (7) traditional eating, (8) natural concerns, (9) sociability, (10) price, (11) visual appeal, (12) weight control, (13) affect regulation, (14) social norms, and (15) social image. An additional sheet with further explanations was given to the respondents. A translated version of the information sheet is given below.

\section{Information Sheet for task 1 according to RENNER et al. (2012)}

1. Appetite

2. Habit

3. Hunger

4. Health

5. Simplicity

6. Pleasure

7. Traditions

8. Naturalness

9. Community

10. Prize

11. Presentation

12. Weight control

13. Affect regulation

14. Social norm
Refers to the tastiness of the components / ingredients assigned to the diet.

Refers to the routine practice of the dietary style.

Refers to the physiological need to provide the body with the energy it needs through this diet.

Refers to the effect of diet on personal health.

The diet can be implemented with little effort.

Refers to the feeling of joy about practicing this diet.

Refers to cultural aspects and customs around diet.

Refers to the ethical aspects concerning the diet.

Refers to social affiliation through the practiced diet.

Monetary aspects associated with the diet.

Refers to the visual stimulus provided through the diet. Contains the dish itself but also the food preparation

Refers to the effect of the diet on body weight.

Refers to the need to reduce emotional stress through dietary intake.

Refers to the expectations of third parties regarding their personal diet. 
15. Social image

Refers to the positive social feedback that is stimulated by the diet.

\section{A.2.2 Pictures Task 1}
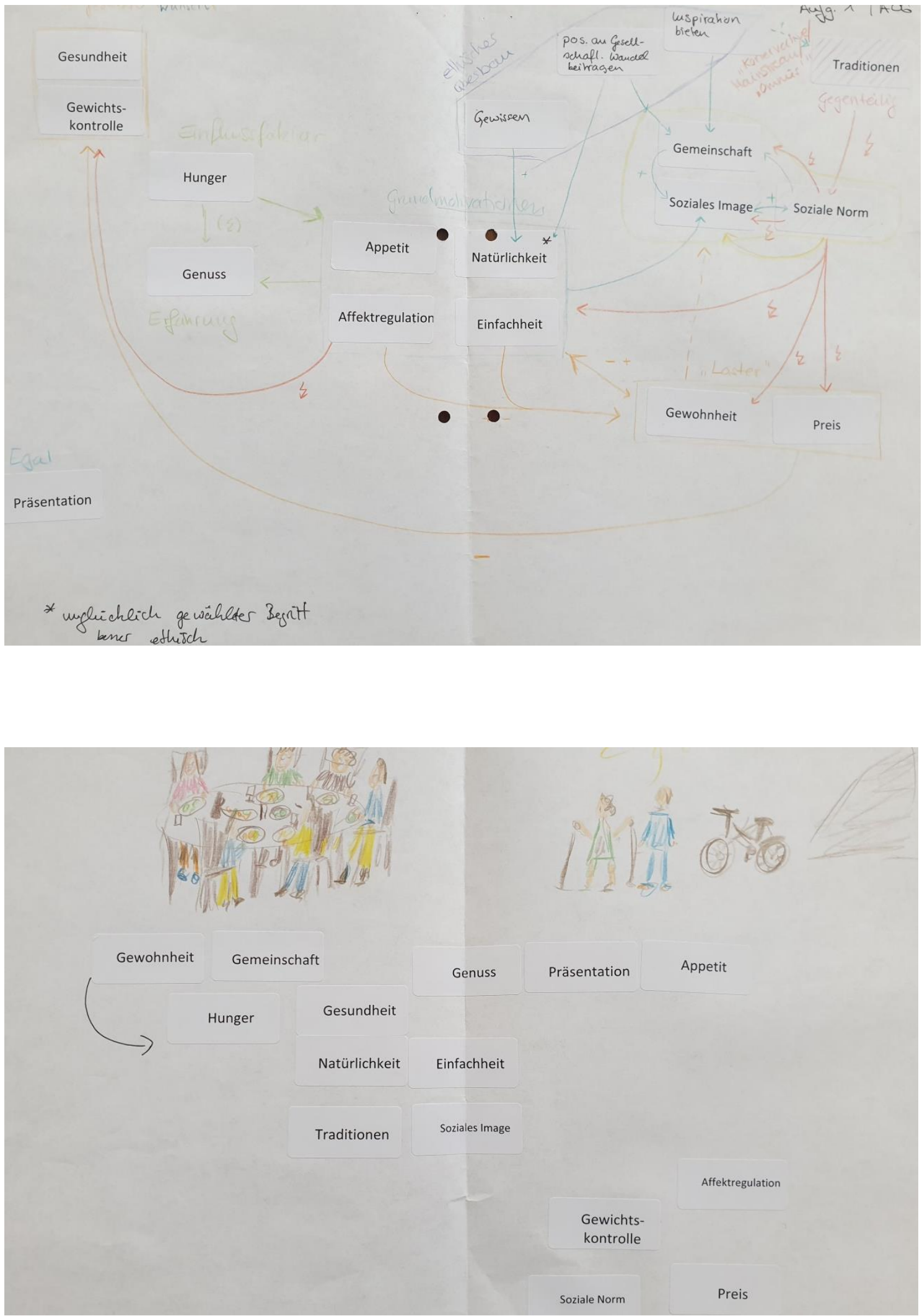


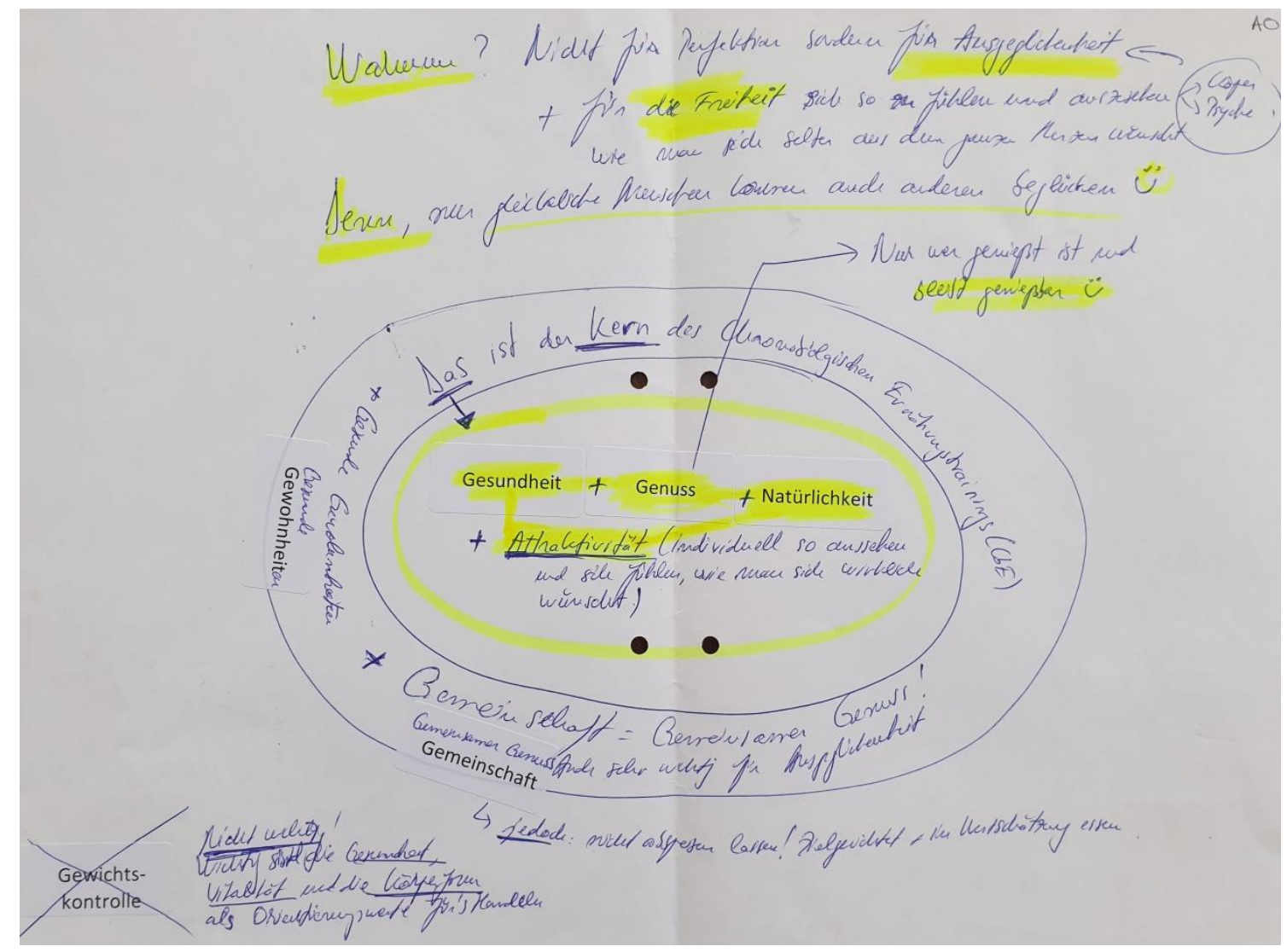




\section{A.3 Task 2: Mental Models}

\section{A.3.1 Questionnaire items}

\begin{tabular}{|c|c|c|c|c|}
\hline Nr. & Scale & Sub scale & Type & Question \\
\hline 1 & Worldview & Worldview & Invers & Most days the world is full of beautiful things \\
\hline 2 & Worldview & Worldview & Invers & l've learned to fully enjoy life. \\
\hline 3 & Worldview & Worldview & direct & It's impossible to feel safe and secure in this life. \\
\hline 4 & Worldview & Meaning of Life & Invers & Life makes sense and that's why it has to be lived. \\
\hline 5 & Worldview & Conviction & Invers & My spiritual convictions have helped me in difficult times. \\
\hline 6 & Worldview & Conviction & direct & I don't believe in ideologies, or if I do they haven't helped me in difficult times. \\
\hline 12 & Idea of man & Experience & Invers & $\begin{array}{l}\text { It helps me to talk about the grave experiences of my life like sicknesses, } \\
\text { accidents, or losses with those I care about. }\end{array}$ \\
\hline 13 & Idea of man & Experience & direct & What isn't talked about ends up being forgotten and stops being painful. \\
\hline 14 & Idea of man & Experience & direct & Talking about things relieves suffering. \\
\hline 15 & Idea of man & Suffering & Invers & You learn from suffering. \\
\hline 16 & Idea of man & Suffering & direct & Suffering is useless pain. \\
\hline 17 & Idea of man & Suffering & direct & Even when there is suffering there can be moments of happiness. \\
\hline 18 & Idea of man & Philanthropy & Invers & I believe that in this world good wins. \\
\hline 19 & Idea of man & Philanthropy & Invers & People help those close to them whenever they can. \\
\hline 20 & Idea of man & Philanthropy & direct & I believe that in this world evil wins. \\
\hline 21 & Idea of man & Philanthropy & direct & People don't usually help those close to them. \\
\hline 22 & Idea of man & Trust & Invers & I tend to trust people. \\
\hline 23 & Idea of man & Communication & direct & Some things are too horrible for words to describe. \\
\hline 24 & Idea of man & Dreams & Invers & $\begin{array}{l}\text { I don't usually remember my dreams and if I remember them I don't usually find } \\
\text { them important. }\end{array}$ \\
\hline
\end{tabular}


Task 2: Questions VIVO (PÉREZ-SALES et al. 2012), Part II

\begin{tabular}{|c|c|c|c|c|}
\hline Nr. & Scale & Sub scale & Type & Question \\
\hline 25 & Confrontation & Objectivity & Invers & I feel like I break everything I touch. \\
\hline 26 & Confrontation & Objectivity & direct & $\begin{array}{l}\text { When I try not to think about something that worries me, I end up thinking about it } \\
\text { more. }\end{array}$ \\
\hline 27 & Confrontation & Active coping & Invers & $\begin{array}{l}\text { When something serious happens to me I tend to think calmly and coolly about } \\
\text { how to solve it. }\end{array}$ \\
\hline 28 & Confrontation & Active coping & direct & Fear stops me from doing things that I used to be able to do. \\
\hline 29 & Confrontation & Memory & direct & You don't choose to forget. \\
\hline 7 & Attitude towards life & Suicide & Invers & I don't believe that one should ever give up on life. \\
\hline 8 & Attitude towards life & Suicide & direct & Suicide is a dignified option that I could come to considered. \\
\hline 9 & Attitude towards life & Destiny & direct & Our lives are predetermined by destiny. \\
\hline 10 & Attitude towards life & Dealing with Ambiguity & direct & It calms me to think about why things happen. \\
\hline 11 & Attitude towards life & Quest for meaning & Invers & Life is about accepting uncertainty and ambiguity. \\
\hline 30 & Past experiences & Guilt feeling & invers & I believe than even the worst feelings of guilt can be forgiven. \\
\hline 31 & Past experiences & Guilt feeling & direct & $\begin{array}{l}\text { I am constantly thinking that l'd be happy if I could only fix the } \\
\text { damage l've done. }\end{array}$ \\
\hline 32 & Past experiences & Guilt feeling & direct & I've made mistakes in the past that I can't bear to remember. \\
\hline 33 & Past experiences & Self-confidence & Invers & I believe that when others assign me responsibilities, they are in good hands. \\
\hline 34 & Past experiences & Self-confidence & direct & The mistakes l've made have made me lose confidence in myself. \\
\hline 35 & Past experiences & Learning effect & Invers & $\begin{array}{l}\text { I believe that l've been through some tough experiences, } \\
\text { which have made me stronger. }\end{array}$ \\
\hline 36 & Past experiences & Learning effect & direct & You never really learn from your mistakes. \\
\hline 37 & Past experiences & Change potential & direct & The way you are never really changes. \\
\hline 38 & Past experiences & Fear & Invers & I always know what my fears are. \\
\hline
\end{tabular}




\section{A.3.2 Pictures Task 2}

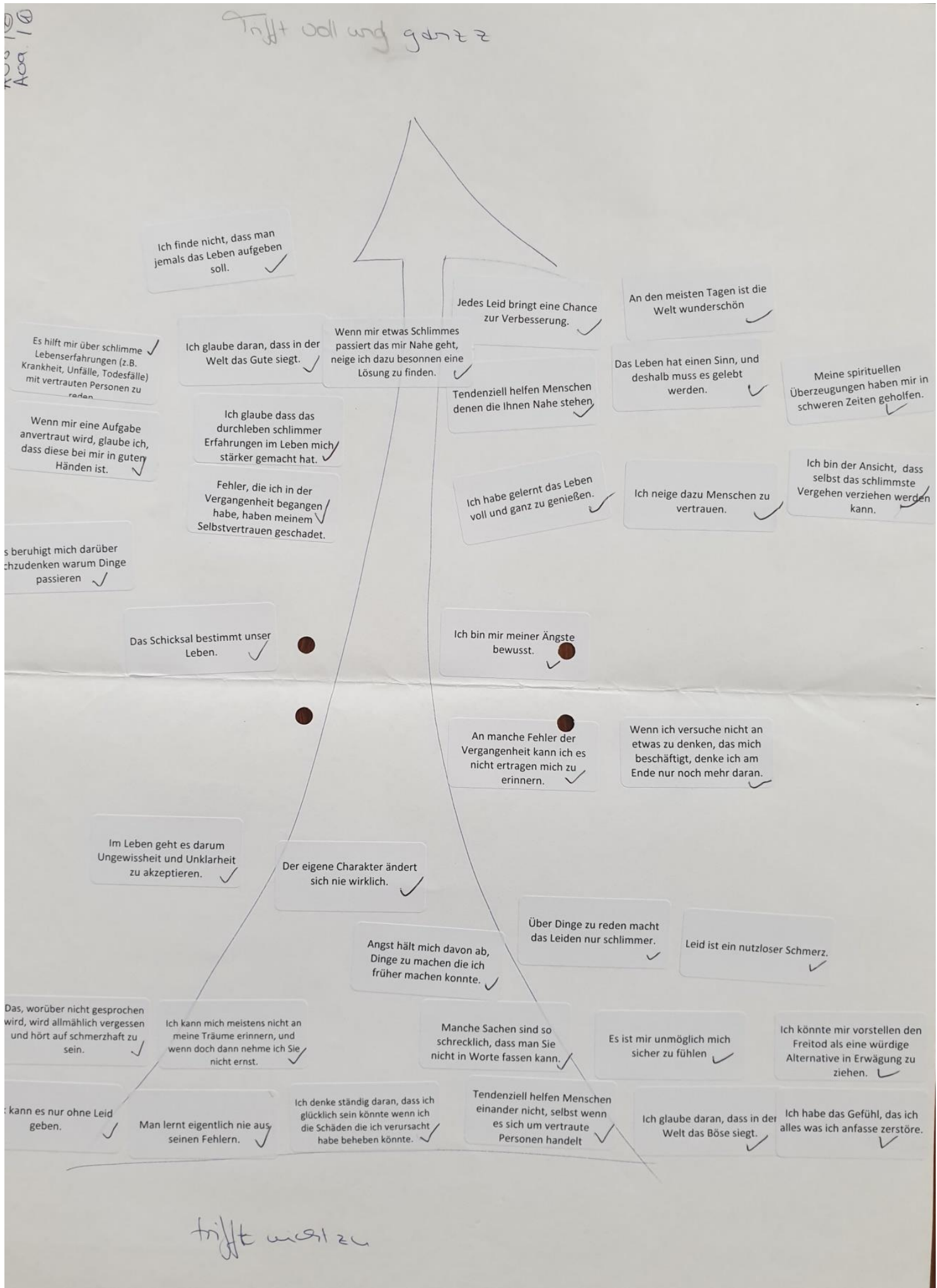


Meine spirituellen

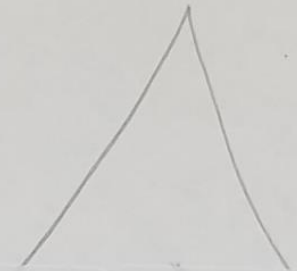

Uberzeugungentuellen

schweren Zeiten Ben mir ir An den meisten Tagen ist dit

Ich habe gelernt das Leben voll und ganz zu genießen.

Das Leben hat einen Sinn, un deshalb muss es gelebt Ich glaube dass das
durchleben schlimmer
Erfahrungen im Leben mich
stärker gemacht hat. $\checkmark$

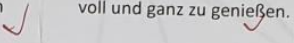
werden. $V$

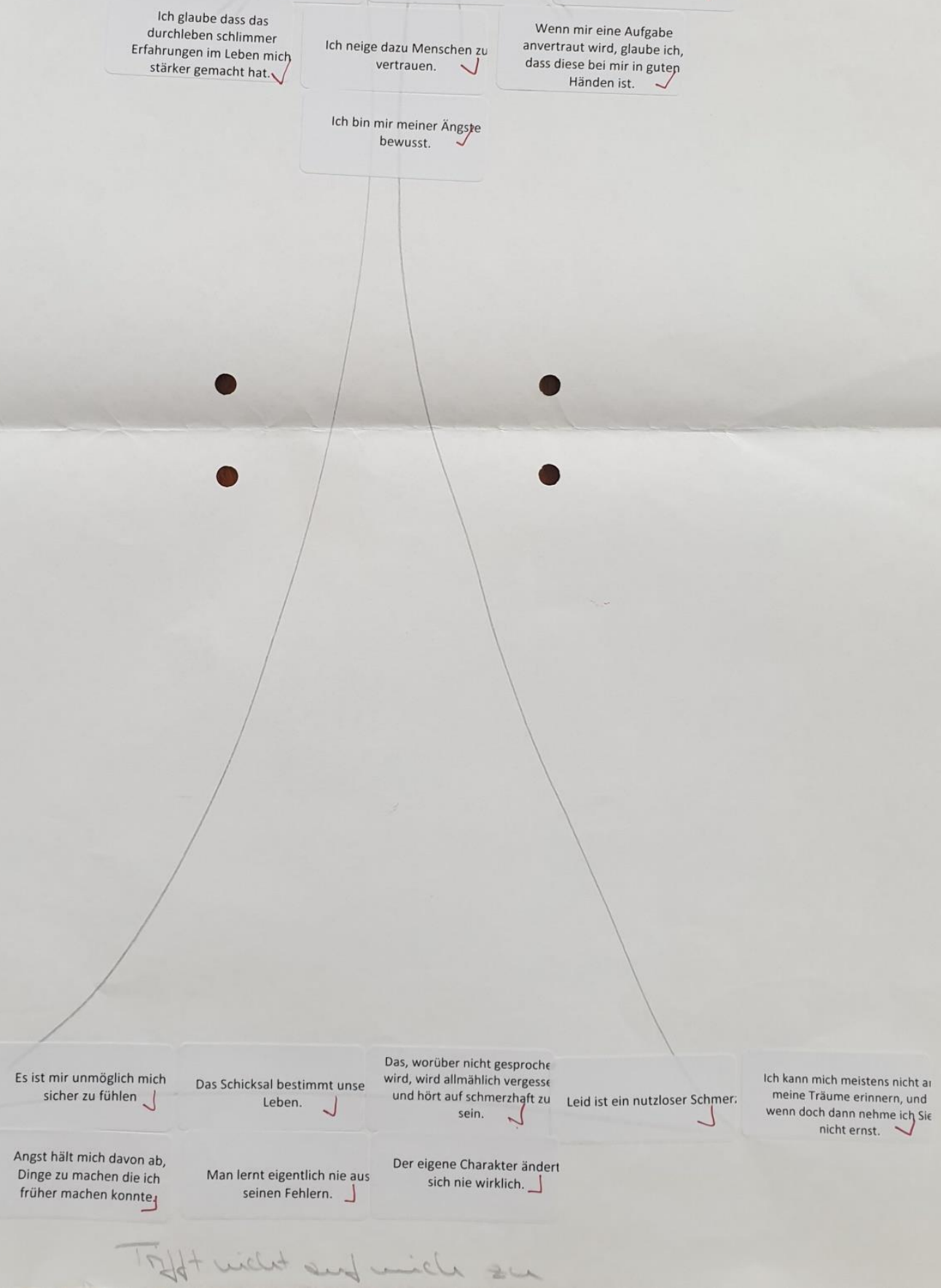




\section{A.4 Questionnaire}

The questionnaire was handed out at the end of the interview. Questions one to seven and eleven to thirteen were choice questions. Question eight was an open question filled out by each respondent. Questions nine and ten included the ranking of each item. Answers were given using a Likert scale ranging from 1 to 5 where 1 indicated a low intensity and 5 indicated a high intensity. 
Questionnaire Essay 1, Part I

\begin{tabular}{|c|c|c|}
\hline Nr. & Question & Option \\
\hline \multirow[t]{3}{*}{1} & Gender & Male \\
\hline & & Female \\
\hline & & Diverse \\
\hline \multirow[t]{2}{*}{2} & Nationality & German \\
\hline & & Other \\
\hline 3 & Year of birth & Open \\
\hline \multirow[t]{7}{*}{4} & Level of education & None \\
\hline & & Certificate of secondary education \\
\hline & & Secondary modem school \\
\hline & & General certificate of secondary education \\
\hline & & Advanced technical college \\
\hline & & Final certificate of secondary education \\
\hline & & Other: \\
\hline \multirow[t]{3}{*}{5} & Household income & Open \\
\hline & & I don't know \\
\hline & & I don't want to answer \\
\hline \multirow[t]{6}{*}{6} & Marital status & Single \\
\hline & & Divorced \\
\hline & & Married and living together \\
\hline & & Married and living separately \\
\hline & & Widow \\
\hline & & I don t want to answer \\
\hline \multirow[t]{7}{*}{7} & Job & $\begin{array}{l}\text { Full-time employment (35 hrs. / week or } \\
\text { more) }\end{array}$ \\
\hline & & Part-time employment (15-34 hrs. / week) \\
\hline & & $\begin{array}{l}\text { Hourly employment (less than } 15 \text { hrs. / } \\
\text { week }\end{array}$ \\
\hline & & Matemity / Paternity leave \\
\hline & & Traineeship / retraining \\
\hline & & $\begin{array}{l}\text { Unemployed (incl. Pupils, students, } \\
\text { pensioners) }\end{array}$ \\
\hline & & I don t want to answer \\
\hline 8 & Diet & Open \\
\hline \multirow[t]{7}{*}{9} & How often in a month do you purchase: & regional / locally produced foods \\
\hline & & Organic foods \\
\hline & & Bio-dynamic foods \\
\hline & & Fair-trade products \\
\hline & & Gen-tech free foods \\
\hline & & Animal welfare characteristics \\
\hline & & $\begin{array}{l}\text { Low degree of processing / } \\
\text { Wholesomeness }\end{array}$ \\
\hline
\end{tabular}




\begin{tabular}{|c|c|c|}
\hline $\mathrm{Nr}$. & Question & Option \\
\hline 10 & $\begin{array}{l}\text { During the past three month my diet was } \\
\text { rich in: }\end{array}$ & $\begin{array}{l}\text { Cereals (e.g. bread, pasta, rice) } \\
\text { Legumes (e.g. beans, chickpeas, lentils) } \\
\text { Refined Sugar (e.g. Sweets, cake, ketchup) } \\
\text { Nuts, Seeds, Oils (e.g. almonds, } \\
\text { pumpkinseeds) } \\
\text { Eggs } \\
\text { Milk and milk products (e.g. butter, yoghurt) } \\
\text { Convenience foods (e.g. frozen foods, } \\
\text { ready-to-eat) } \\
\text { Fresh vegetables } \\
\text { Red meat (e.g. cattle) } \\
\text { Other meats (e.g. pork, chicken) } \\
\text { Processed meats (e.g. sausages) } \\
\text { Fish and sea foods } \\
\text { Fresh fruits } \\
\text { Saturated fats (e.g. fried foods, baked } \\
\text { goods) } \\
\text { Soft drinks (e.g. lemonade) }\end{array}$ \\
\hline 11 & Cultivation of crops & $\begin{array}{l}\text { Yes } \\
\text { No }\end{array}$ \\
\hline 12 & $\begin{array}{l}\text { If yes, how much of your dietary intake is } \\
\text { covered? }\end{array}$ & $\begin{array}{l}\text { Less than } 10 \% \\
10-25 \% \\
26-50 \% \\
51-75 \% \\
\text { More than } 75 \%\end{array}$ \\
\hline 13 & $\begin{array}{l}\text { If yes, for what reason do you cultivate } \\
\text { crops? }\end{array}$ & $\begin{array}{l}\text { Financial aspects } \\
\text { Routine / joy / pleasure } \\
\text { Long distance to markets } \\
\text { Important contribution to family nutrition } \\
\text { Other: }\end{array}$ \\
\hline
\end{tabular}




\section{Appendix B}

\section{B.1 Questionnaire}

In the original questionnaire the items of different constructs were placed randomly. However, for a better overview the following section provides an organized presentation of the different constructs of interest utilized within the study.

\section{Part 0: Welcome page}

Dear participant,

thank you for taking the time to answer our questions.

This questionnaire is part of a research project of the University of Goettingen, Germany in which we intend to capture determinants that influence dietary behaviour. In total, this survey will take approx. 20-25 minutes.

Our diets are a very personal reflection of identity. We are very grateful that you share this information with us as it will support us in gaining valuable insights on food consumption behaviour. Please be assured that your information is handled with the highest care and will be kept confidential. Also, it will not be passed on to a third party and will not be processed for any other purpose than this research.

Your participation is highly appreciated.

In this sense, let's get started...

\section{Part 1: Demographics A}

1. Which year were you born in? [Drop-down list]

2. What is your gender? [Male / female/ diverse]

3. What is the highest level of education you completed?

India: [none / primary school / middle school / higher secondary school / senior school / bachelor degree / master degree / postgraduate degree / other]

USA: [none / less than high school / some high school / high school / some college / associate's / bachelor degree / master degree / postgraduate degree / other] 
4. What is your combined monthly household income? (By household we refer to a group of people that live under the same roof. Please indicate or estimate the amount of money available after taxes have been paid.)

India: $[<25.000$ / 25.000-34.999 / 35.000-49.999 / 50.000-74.999 / 75.000-99.999 / 100.000-150.000/ > 150.000] [INR]

USA: $[<60.000$ / 60.000-89.999 / 90.000-119.999 / 120.000-239.999 / 240.000-599.999 / 600.000-1,200.000 / > 1,200.000] [USD]

\section{Part 2: Food Choice and Dietary Behaviour Part I}

Characteristics: Never true | rarely true | sometimes true | often true | always true

Q: How true are the following statements for you?

5. I consciously choose food that supports me in staying healthy.

6. For my meals, I alternate between different food groups.

7. I combine food from different food groups to meet my dietary needs.

8. I feel sleepy bloated, and/or heavy after eating.

9. I only eat when I am hungry.

10. I consciously choose food that is low in sugar.

11. I use frozen foods.

12. I eat meat for the main meals.

13. When buying fresh fruits / veggies, I consciously choose old/traditional varieties.

14. I choose food that meets my personal quality criteria regardless of its price.

15. When purchasing groceries I pick the products with the cheapest price.

16. I buy imported fruits from overseas.

17. I buy food that was produced within the region I stay.

18. I refrain from food with excessive packaging.

19. I consciously buy the fruits and vegetables that are in season.

20. I overeat during meals.

21. When cooking meals, I only prepare the amount of food that will be eaten.

22. I follow religious guidelines when preparing food.

\section{Part 3: Pro-environmental Behaviour}

Characteristics: Never true | rarely true | sometimes true | often true | always true

Q: How true are the following statements for you?

23. I limit the amount of water I use in order to conserve it (e.g. while showering, cleaning, dish washing). 
24. I wait until I have a full load before I use the washing machine, dish washer, etc.

25. I participate in a pro-environmental, conservation, or wildlife protection group.

26. I contribute money to environmental, conservation, or wildlife protection groups.

27. During the last few months, I limited the amount of meat I consumed.

28. During the last few months, I limited the amount of dairy products I consumed (e.g. cheese, yoghurt, milk).

29. I use public transportation.

30. I walk or cycle instead of using other means of transportation.

\section{Part 4: Mindfulness}

Characteristics: Never true | rarely true | sometimes true I often true | always true

Q: How true are the following statements for you? Please consider the statements based on your experience over the last three month

31. When my mood changes I notice it right away.

32. I am able to sense which feelings I experience when talking to other people (e.g. if I am delighted or sad).

33. I judge myself harshly when I make a mistake.

34. I guide myself with loving kindness through the ups and downs of life.

35. When I make life unnecessarily difficult for myself, I become aware of it shortly after.

36. When I notice that I have made things more complicated than they really are, I can smile about it.

37. During the day, I am aware of the fact that many thoughts are my personal interpretations and do not necessarily reflect the reality.

38. I am aware of the fact that my judgements about situations and persons can change easily.

39. When I am tangled up in stressful thoughts and feelings, I notice this quickly and can easily dissociate myself from them.

40. I notice my thoughts and feelings, yet I am able to look at them from a distance.

41. While reading, I often have to re-read sections because I go distracted by my thoughts.

42. In the everyday life, I often get distracted by memories, images or dreams.

43. I notice details in nature (e.g. colour of the sky...).

44. I consciously notice sounds around me, such as birds chirping or cars passing.

45. When I notice that I have made things more complicated than they really are, I can smile about it.

46. During the day, I am aware of the fact that many thoughts are my personal interpretations and do not necessarily reflect the reality. 


\section{Part 5: Spirituality}

Characteristics: not at all $\mid$ hardly at all $\mid$ somewhat $\mid$ to a reasonable degree $\mid$ to a high degree

Q: To what extend do the following statements generally apply to you?

47. I approach the world with trust.

48. In difficult times, I maintain my inner peace.

49. It is important to me that I can do things for others.

50. I try to make a meaningful contribution to society.

51. My life has meaning and purpose.

52. I experience the things I do as meaningful.

53. The beauty of nature moves me.

54. When I am in nature, I feel a strong sense of connection.

55. I accept that I am not able to influence everything.

56. I am aware of the fact that each life has its own tragedy.

57. There is a God or higher power in my life that gives me guidance.

58. I meditate or pray, or take time in other ways to find inner peace.

59. I have had experiences in which all things seemed to be part of a greater whole.

60. I have had experiences in which I seemed to merge with a power greater than myself.

\section{Part 6: Food Choice and dietary behaviour Part 2}

61. Are you currently following any particular diet? [Yes / No]

62. If yes, what kind of diet do you practice?

a. I eat eggs and dairy-products but no meats, chicken, or fish/seafood

b. I eat dairy product but no eggs, meats, chicken, or fish/seafood

c. I don't eat any product that is derived from animal sources (no eggs, no dairy products, no meats, no chicken, no fish, no honey)

d. I only eat food that has not been heated above $48{ }^{\circ} \mathrm{C}$ (raw foodism)

e. I combine foods according to their digestive requirements (food combining)

f. I only eat unprocessed and unrefined foods (wholefoods diet)

g. I follow a paleolithic diet (meat and vegetable based; no dairy products, grains, sugar, legumes, processed oils, salt, alcohol, and coffee)

h. Other:

63. How often do you purchase groceries with the following attributes?

Characteristic: never | rarely | sometimes | often | always 

a. Locally produced.
b. Organic certification.
c. Bio-dynamic certification (Demeter label).
d. Fair trade certification.
e. GMO free.
f. Animal welfare characteristics (e.g. free-range, animal welfare approved, certified humane).
g. Low degree of Processing / naturalness of product.
h. Wholesomeness of product (e.g. whole grain flour products, wholegrain rice).
i. Other attribute that is important to me but was not mentioned above:

64. If I consider my dietary behaviour throughout the 3 month I can say that my diet is rich in...

Characteristic: not part of my diet | low amount I medium amount | high amount I part of my daily diet
a. Grains / cereals (e.g. bread, pasta, rice).
b. Legumes (e.g. beans, lentils, chickpeas, peanuts, peas).
c. Refined sugar (e.g. coke, regular sweets, ketchup, cornflakes, jam).
d. Nuts, seeds, and oils (e.g. almonds, walnut, sesame seeds, pumpkin seeds, vegetable oil).
e. Eggs.
f. Dairy products (e.g. milk, butter, yoghurt, cheese).
g. Convenience foods (e.g. ready-to-eat products, frozen foods, prepared mixes).
h. Unprocessed Vegetables.
i. Red meat.
j. $\quad$ Other meats (e.g. sheep, poultry, pork).
k. Processed meats (e.g. salami, sausage).
I. Fish / Sea food.
m. Nuts, seeds, and oils.
n. Unprocessed Fruits.
o. Saturated fats (e.g. regular-fat milk and cheese, sausages, meats, pastries, cakes, deep fried food).

\section{Part 7: Additional information}

65. Do your grow/produce some of the food you consume? [Yes / no]

66. If yes, what is the reason for that?
a. Expensive price of market food.
b. Joy / pleasure / hobby.
c. Long distance to market.
d. Necessary support to household nutrition.
e. Quality issues of market food. 
67. Please indicate the degree to which the food you produce / grow contributes to family nutrition? [less than 10\%/ 10-25 \%/26-50\%/51$75 \% /$ more than $75 \%]$

68. Did you grow up in a family that actively practiced any religion (e.g. following religious rules, regular church visits)

69. What describes best the religion you were raised in? [Agnosticism / Atheism / Buddhism / Christianity / Hinduism / Islam / Judaism / spiritual but not religious / Taoism]

70. Do you actively practice any religion? [Yes / No]

71. What describes best your current religion? [Agnosticism / Atheism / Buddhism / Christianity / Hinduism / Islam / Judaism / spiritual but not religious / Taoism]

72. Most of my knowledge about nutrition and food preparation derives from:

a. the family member/person who prepared/cooked food during my childhood.

b. information I gathered through books, internet, or other sources.

c. own experimentation with different food lifestyles, cooking styles, diets.

d. From a specialist (dietitian, doctor, etc.).

e. From a close family member / spouse / friend.

73. I consider myself a religious person [not at all \ hardly at all \somewhat $\backslash$ to a reasonable degree $\backslash$ to a high degree]

74. I consider myself a spiritual person [not at all $\backslash$ hardly at all $\backslash$ somewhat $\backslash$ to a reasonable degree $\backslash$ to a high degree]

\section{Part 8: Demographics B}

75. What is your nationality? [Drop-down list]

76. What describes you living condition best? [Live by myself / live with partner / live with family / live with sharing community / live in a boarding situation]

77. What describes the main occupation of the household head best? (By household head we refer to the person who is responsible for the organization and care of your household or who is regarded as such by the members of your household. Generally, this person also provides the chief source of income and decides about how it is spent.) [Employment, full time / employment, part-time / self-employed / unemployed / house person or care taker / student / pensioner / seasonal worker] 
78. In what kind of settlement do you live? [Rural / peri or sub-urban / urban]

79. I believe religion and spirituality are inextricably linked [Yes / no]

80. If no, what do you think is the difference between spirituality and religion? Please explain your thoughts in 1-3 sentences. [Open]

\section{Part 9: Last page}

Dear participant.

Again, thank you very much for your participation. Your input is much appreciated, we feel thankful that you took the time to support us in our research.

May you have a beautiful day. 


\section{Appendix C}

\section{C.1 Definitions of Mindfulness in the Literature}

Overviewing the various definitions of mindfulness part I

\begin{tabular}{|c|c|c|c|}
\hline Nr. & Year & Author & Definition \\
\hline 1 & 1852 & Palsgrave & $\begin{array}{l}\text { "Myndfulnesse, pencee' according to Oxfrod English } \\
\text { Dictionary: Mindfulness in the sense of 'the quality or } \\
\text { state of being conscious or aware of something; } \\
\text { attention. Also: tmemory (obsolete); tintention, } \\
\text { purpose (obsolete)' }\end{array}$ \\
\hline 2 & 1872 & Monier-Williams & $\begin{array}{l}\text { (1) "smrti" remembrance, memory, reminiscence, } \\
\text { recollection, thinking of or upon (any person or thing), } \\
\text { calling to mind. (2) "smarati" (verb) to remember, to } \\
\text { recollect, call to mind, bear in mind, think of, think upon, } \\
\text { be mindful of. }\end{array}$ \\
\hline 3 & 1875 & Childers & $\begin{array}{l}\text { (1) "sati" recollection, active state of mind, fixing the } \\
\text { mind strongly upon any subject, attention, } \\
\text { attentiveness, thought, reflection, and } \\
\text { consciousness. (2) "upatthita satihe" presence of } \\
\text { mind. (3) "sati patthāna" fixing the attention, earnest } \\
\text { meditation. }\end{array}$ \\
\hline 4 & 1881 & Rhys Davids & $\begin{array}{l}\text { (1) "sati": mental activity, thought. (2) "satipattha' nas": } \\
\text { earnest meditations. ( } 3 \text { ) "samma-sati": sati is literally } \\
\text { 'memory,' but is used with reference to the constantly } \\
\text { repeated phrase 'mindful and thoughtful' (sato } \\
\text { sampaja' no); and means that activity of mind and } \\
\text { constant presence of mind which is one of the duties } \\
\text { most frequently inculcated on the good Buddhist. }\end{array}$ \\
\hline 5 & 1989 & Langer & $\begin{array}{l}\text { open to novelty, being sensitive to content and } \\
\text { perspective, creating new categories, changing } \\
\text { mindsets, challenging, assumptions, breaking set, } \\
\text { getting involved, and taking, responsibility. }\end{array}$ \\
\hline
\end{tabular}


Nr. Year Author Definition

61994 Kabat-Zinn "Paying attention in a particular way: on purpose, in the present moment, and non-judgmentally."

71999 Marlatt, Kristeller

"...bringing one's complete attention to the present experience on a moment-to-moment basis."

82003 Brown, Ryan

"Mindfulness is the state of being attentive to and aware of what is taking place in the present."

"...two component model of mindfulness: The first component involves the self-regulation of attention so that it is maintained on immediate experience, thereby allowing for increased recognition of mental events in

92004 Bishop et al. the present moment. The second component involves adopting a particular orientation toward one's experiences in the present moment, an orientation that is characterized by curiosity, openness, and acceptance."

"Mindfulness is awareness and the ability to see the happenings of one's inner and outer worlds. Mindfulness can be viewed as an ongoing process of expanding one's awareness to include stimuli that 102004 Rosenberg might otherwise be filtered out or not attended to, of becoming aware of the kinds of biases to which one's mind might typically be vulnerable, and of maintaining a nonjudgmental stance toward what arises in one's own mind (including emotions as well as sensations provided from one's own body and the outside world)."

"A quality of consciousness that denotes a receptive 112005 Brown, Kasser attention to and awareness of ongoing internal states and behavior, mindfulness promotes subjective wellbeing and ecologically responsible behavior." 
Nr. Year

122006

132006

$142007 \quad$ Jha et al. “...five components of Mindfulness: Observe, Describe, Act with Awareness, Nonjudge, and Nonreact."

"...three components (axioms) of mindfulness:

"... an attention that is receptive to the whole field of awareness and remains in an open state so that it can be directed to currently experienced sensations, thoughts, emotions, and memories."

"Mindfulness can be considered a universal human capacity proposed to foster clear thinking and openheartedness. As such, this form of meditation requires no particular religious or cultural belief system.

152008 Ludwig, Kabat-Zinn The goal of mindfulness is to maintain awareness moment by moment, disengaging oneself from strong attachment to beliefs, thoughts, or emotions, thereby developing a greater sense of emotional balance and well-being."

162009 Jacob et al “...non-judgmental awareness in the present moment"

"... as the tendency to be highly aware of one's internal

172008 Cardaciotto et al. and external experiences in the context of an accepting, nonjudgmental stance towards those experiences."

“...an awareness that arises through intentionally 182009 Shapiro, Carlson attending in an open, accepting, and discerning way to whatever is arising in the present moment."

"...attention and awareness of internal and external phenomena." 
Nr. Year

Author

Definition

202010 Gilbert et al.

212011

Mikulas

222011 Delle Fave et al

$232011 \quad$ Schmidt

$242012 \quad$ Papies et al.

$252013 \quad$ Hunecke

262014 Ericson et al.

272015

Lengyel
"Mindfulness involves nonjudgmental awareness of one's present experience."

"...the behavior of the mind that I above call "awareness" and elsewhere call "mindfulness." Mindfulness, as a behavior of the mind, is the active maximizing of the breadth and clarity of awareness. It includes moving and sharpening the focus of awareness within the field of consciousness"

“... ability to filter and consciously select which interoand exteroceptive information to attend to and construe meaning from. It comprises both, eliminative and generative mechanisms"

"...a state of awareness of the present moment with a certain breadth of the mind in which one tries to observe without interfering."

“...method for facilitating self-regulation, mindfulness procedure: observation of reactions to external stimuli as transient mental events rather than subjectively real experiences."

"...intent, attentiveness to the present moment, and a non-judgmental mindset mindfulness describes both a process and an outcome"

“...present-moment awareness that includes everything that happens within the individual and in the surrounding environment."

"Universal and effective instrument to catalyze a paradigm shift both at the individual and the collective level. Witnessing, non-attachement and enlightement are important aspects of mindfulness that are often neglected" 
282015 Barbaro, Picket "...heightened awareness and attention to the present moment, in both experience and behavior."

$292017 \quad$ Tezel, Giritli “...a form of behavioral regulation.”

$302017 \quad$ Fischer et al.

“...deliberate, unbiased and openhearted awareness of perceptible experience in the present moment focus on cultivation of benevolent and clear-headed values and actions to self, others and the world, and on the value in fostering greater coherence between values, attitudes and behavior."

“...nonjudgmental awareness of the present moment, 312018 Hosey et al. which provides an opportunity to acknowledge and accept difficult physical and emotional sensations."

"...non-evaluative attitude, paying attention to the 322019 Hunecke, Richter present moment, leave the "automatic pilot" mode and decrease reactive behavior." 


\section{C.2 Reference list of appendix C.1}

[1] Palsgrave, J. (1852). 1530. L'Eclaircissement de la langue française. Paris: Imprimerie Nationale.

[2] Monier-Williams, M. (1872). A Sanskrit-English Dictionary Etymologically and Philologically Arranged: With Special Reference to Greek, Latin, Gothic, German, Anglo-Saxon, and Other Cognate Indo-European Languages. Clarendon Press.

[3] Childers, R. C. (1875). A dictionary of the Pa 1i language. London: Trübner \& Co.

[4] Rhys Davids, T. W. (1881). Buddhist suttas. Oxford: Clarendon Press.

[5] Langer, E. J. (1989).Mindfulness. Reading: Addison-Wesley.

[6] Kabat-Zinn, J. (1994).Wherever you go, there you are: Mindfulness meditation in everyday life. NewYork: Hyperion.

[7] Marlatt, G. A., \& Kristeller, J. L. (1999). Mindfulness and meditation. In: W. R. Miller (Ed.), Integrating spirituality into treatment: Resources for practitioners (pp. 67-84). Washington, DC: American Psychological Association.

[8] Brown, K. W. \& Ryan, R. M. (2003). The benefits of being present: Mindfulness and its role in psychological well-being. Journal of Personality and Social Psychology, 84, 822-848.

[9] Bishop, S. R., Lau, M., Shapiro, S., Carlson, L., Anderson, N. D., Carmody, J., ... \& Devins, G. (2004). Mindfulness: A proposed operational definition. Clinical psychology: Science and practice, 11(3), 230-241.

[10] Rosenberg, E. L. (2004). Mindfulness and consumerism. In T. Kasser \& A. de Kanner (Eds), Psychology and consumer culture. The struggle for a good life in a materialistic world (pp. 107-125). Washington, DC: American Psychological Association.

[11] Brown, K. W., \& Kasser, T. (2005). Are psychological and ecological well-being compatible? The role of values, mindfulness, and lifestyle. Social indicators research, 74(2), 349-368.

[12] Baer, R. A., Smith, G. T., Hopkins, J., Krietemeyer, J., \& Toney, L. (2006). Using self-report assessment methods to explore facets of mindfulness. Assessment, 13(1), 27-45.

[13] Shapiro, S. L., \& Carlson, L. E. (2009). The art and science of mindfulness: Integrating mindfulness into psychology and the helping professions. American Psychological Association.

[14] Jha, A. P., Krompinger, J., \& Baime, M. J. (2007). Mindfulness training modifies subsystems of attention. Cognitive, Affective, \& Behavioral Neuroscience, 7(2), 109-119. 
[15] Ludwig, D. S., \& Kabat-Zinn, J. (2008). Mindfulness in medicine. Jama, 300(11), 1350-1352.

[16] Jacob, J., Jovic, E., \& Brinkerhoff, M. B. (2009). Personal and planetary well-being: Mindfulness meditation, pro-environmental behavior and personal quality of life in a survey from the social justice and ecological sustainability movement. Social Indicators Research, 93(2), 275-294.

[17] Cardaciotto, L., Herbert, J. D., Forman, E. M., Moitra, E., \& Farrow, V. (2008). The assessment of present-moment awareness and acceptance: The Philadelphia Mindfulness Scale. Assessment, 15(2), 204-223.

[18] Shapiro, S. L., \& Carlson, L. E. (2009). The art and science of mindfulness: Integrating mindfulness into psychology and the helping professions. American Psychological Association.

[19] Amel, E. L., Manning, C. M., \& Scott, B. A. (2009). Mindfulness and sustainable behavior: Pondering attention and awareness as means for increasing green behavior. Ecopsychology, 1(1), 14-25.

[20] Gilbert, D., \& Waltz, J. (2010). Mindfulness and health behaviors. Mindfulness, 1(4), 227-234.

[21] Mikulas, W. L. (2011). Mindfulness: Significant common confusions. Mindfulness, 2(1), 1-7.

[22] Delle Fave, A., Massimini, F., \& Bassi, M. (2011). Psychological selection and optimal experience. In Psychological Selection and Optimal Experience Across Cultures (pp. 39-58). Springer, Dordrecht.

[23] Schmidt, S. (2011). Mindfulness in east and west-is it the same?. In H. Walach, S. Schmidt, \& W. B. Jonas (Eds.), Neuroscience, consciousness and spirituality (pp. 23-38). Dordrecht: Springer.

[24] Papies, E. K., Barsalou, L. W., \& Custers, R. (2012). Mindful attention prevents mindless impulses. Social Psychological and Personality Science, 3(3), 291-299.

[25] Hunecke, M. (2013). Psychological resources for sustainable lifestyles. A report for Denkwerk Zukunft-Foundation for cultural renewal.

[26] Ericson, T., Kjønstad, B. G., \& Barstad, A. (2014). Mindfulness and sustainability. Ecological Economics, 104, 73-79.

[27] Lengyel, A. (2015). Mindfulness and sustainability: utilizing the tourism context. Journal of Sustainable Development, 8(9), 35.

[28] Barbaro, N., \& Pickett, S. M. (2016). Mindfully green: Examining the effect of connectedness to nature on the relationship between mindfulness and engagement in pro-environmental behavior. Personality and Individual Differences, 93, 137-142. 
[29] Tezel, E., \& Giritli, H. (2017, March). Understanding sustainability through mindfulness: A systematic review. In International Sustainable Buildings Symposium (pp. 321-327). Springer, Cham.

[30] Fischer, D., Stanszus, L., Geiger, S., Grossman, P., \& Schrader, U. (2017). Mindfulness and sustainable consumption: A systematic literature review of research approaches and findings. Journal of Cleaner Production, 162, 544-558.

[31] Hosey, M., McWhorter, J. W., \& Wegener, S. T. (2018). Psychologic interventions for chronic pain. In Essentials of pain medicine (pp. 539-544). Elsevier.

[32] Hunecke, M., \& Richter, N. (2019). Mindfulness, construction of meaning, and sustainable food consumption. Mindfulness, 10(3), 446-458. 


\section{Bibliography}

Aerts, D., Apostel, L., De Moor, B., Hellemans, S., Maex, E., Van Belle, H., \& Van der Veken, J. (1994). World views: From fragmentation to integration. Brussels: VUBPress.

Afsar, B., Badir, Y., \& Kiani, U. S. (2016). Linking spiritual leadership and employee pro-environmental behavior: The influence of workplace spirituality, intrinsic motivation, and environmental passion. Journal of Environmental Psychology, 45, 79-88.

Aitken, D. T. (2016). Experience and morality: Buddhist ethics as moral phenomenology (Doctoral dissertation, University of Tasmania). Retrieved June 16th, 2020 from: https://eprints.utas.edu.au/23404/

Ajzen, I. (1991). The theory of planned behavior. Organizational behavior and human decision processes, 50(2), 179-211.

Aleksandrowicz, L., Green, R., Joy, E. J., Smith, P., \& Haines, A. (2016). The impacts of dietary change on greenhouse gas emissions, land use, water use, and health: a systematic review. PloS one, 11(11), e0165797.

Allirot, X., Miragall, M., Perdices, I., Baños, R. M., Urdaneta, E., \& Cebolla, A. (2018). Effects of a brief mindful eating induction on food choices and energy intake: external eating and mindfulness state as moderators. Mindfulness, 9(3), 750-760.

Allport, G. W. (1963). Behavioral science, religion, and mental health. Journal of religion and health, 187-197.

Alsaffar, A. A. (2016). Sustainable diets: The interaction between food industry, nutrition, health and the environment. Food science and technology international, 22(2), 102-111.

Amel, E. L., Manning, C. M., \& Scott, B. A. (2009). Mindfulness and sustainable behavior: Pondering attention and awareness as means for increasing green behavior. Ecopsychology, 1(1), 14-25.

Armstrong, A. (2012). Mindfulness and consumerism: A social psychological investigation. University of Surrey (United Kingdom).

Arnould, E. J., Price, L. L., \& Otnes, C. (1999). Making magic consumption: A study of white-water river rafting. Journal of contemporary ethnography, 28(1), 33-68.

Atkins, P., \& Bowler, I. (2001). Food Habits, Beliefs and Taboos. Food in Society: Economy, Culture, Geography, 296-310.

Attention. (n.d.) In: Cambridge Dictionary Online. Cambridge Dictionary. Retrieved: June 5th, 2020, from: https://dictionary.cambridge.org/de/worterbuch/englisch/attention. 
Awareness. (n.d.) In: Cambridge Dictionary Online. Cambridge Dictionary. Retrieved: June 5th, 2020, from: https://dictionary.cambridge.org/de/worterbuch/englisch/awareness.

Azur, M. J., Stuart, E. A., Frangakis, C., \& Leaf, P. J. (2011). Multiple imputation by chained equations: what is it and how does it work? International journal of methods in psychiatric research, 20(1), 40-49.

Baer, R. A. (2011). Measuring mindfulness. Contemporary Buddhism, 12(1), 241-261.

Baer, R. A., Smith, G. T., Hopkins, J., Krietemeyer, J., \& Toney, L. (2006). Using selfreport assessment methods to explore facets of mindfulness. Assessment, 13(1), 27-45.

Baer, R. A., Smith, G. T., Lykins, E., Button, D., Krietemeyer, J., Sauer, S., ... \& Williams, J. M. G. (2008). Construct validity of the five facet mindfulness questionnaire in meditating and nonmeditating samples. Assessment, 15(3), 329342.

Baldwin, R. H. \& Blom G.E. (2010). The missing church. The power of being the body of Christ in the family, neighborhood and workplace. London: Tate Publishing.

Ball, D., Hampton, R., Chronis, A., \& Bunker, M. (2001). The development of spirituality and its effect on consumer behavior. In American Marketing Association. Conference Proceedings (Vol. 12, p. 3). American Marketing Association.

Barbaro, N., \& Pickett, S. M. (2016). Mindfully green: Examining the effect of connectedness to nature on the relationship between mindfulness and engagement in pro-environmental behavior. Personality and Individual Differences, 93, 137-142.

Barrett, B., Grabow, M., Middlecamp, C., Mooney, M., Checovich, M. M., Converse, A. K., ... \& Yates, J. (2016). Mindful climate action: health and environmental cobenefits from mindfulness-based behavioral training. Sustainability, 8(10), 1040.

Batat, W., Manna, V., Ulusoy, E., Peter, P. C., Ulusoy, E., Vicdan, H., and Hong, S. (2016). New paths in researching "alternative" consumption and well-being in marketing: alternative food consumption/Alternative food consumption: What is "alternative"?/Rethinking "literacy" in the adoption of AFC/Social class dynamics in AFC. Marketing Theory, 16(4), 561-561.

Belahsen, R. (2014). Nutrition transition and food sustainability. Proceedings of the Nutrition Society, 73(3), 385-388.

Bergomi, C., Tschacher, W., \& Kupper, Z. (2013). Measuring mindfulness: first steps towards the development of a comprehensive mindfulness scale. Mindfulness, 4(1), 18-32.

Birt, C., Buzeti, T., Grosso, G., Justesen, L., Lachat, C., Lafranconi, A., ... \& SarlioLähteenkorva, S. (2017). Healthy and sustainable diets for European countries. Utrecht: European Public Health Association. 
Bishop, S. R., Lau, M., Shapiro, S., Carlson, L., Anderson, N. D., Carmody, J., ... \& Devins, G. (2004). Mindfulness: A proposed operational definition. Clinical psychology: Science and practice, 11(3), 230-241.

Black, D. S. (2011). A brief definition of mindfulness. Behavioral Neuroscience, 7(2), 109.

Boddy, C. R. (2016). Sample size for qualitative research. Qualitative Market Research, 19, 426-432.

Bodhi, B. (1998). Two styles of insight meditation. BPS Newsletter Cover Essay No. 45. Kandy, Sri Lanka: Buddhist Publication Society.

Bodhi, B. (2013). What does mindfulness really mean. A Canonical Perspective. Contemporary Buddhism 12(1), 19-39.

Boudreaux, E. D., Bock, B., \& O'Hea, E. (2012). When an event sparks behavior change: an introduction to the sentinel event method of dynamic model building and its application to emergency medicine. Academic Emergency Medicine, 19(3), 329335.

Bourne, E. J. (2009). Global shift: How a new worldview is transforming humanity. Oakland: New Harbinger Publications.

Bresnahan, M., Zhuang, J., \& Zhu, X. (2016). Why is the vegan line in the dining hall always the shortest? Understanding vegan stigma. Stigma and Health, 1(1), 3-15.

Broglia, A., \& Kapel, C. (2011). Changing dietary habits in a changing world: emerging drivers for the transmission of foodborne parasitic zoonoses. Veterinary parasitology, 182(1), 2-13.

Brown, K. W., \& Kasser, T. (2005). Are psychological and ecological well-being compatible? The role of values, mindfulness, and lifestyle. Social indicators research, 74(2), 349-368.

Brown, K. W., \& Ryan, R. M. (2003). The benefits of being present: mindfulness and its role in psychological well-being. Journal of personality and social psychology, 84(4), 822-848.

Buchanan-Olivier, M., \& Schau, J. S. (2013). Consuming spirituality and the spirituality of consuming media narratives: Why vampirism, why Twilight, why now? In D. Rinallo, L. Scott, \& P. Maclaran (Eds.), Spirituality and consumption (pp. 81-93). New York: Routledge.

Capra, F. (1983). The turning point: Science, society, and the rising culture. Toronto, Ont.: Bantam.

Carmody, J. F. (2014). Eastern and western approaches to mindfulness: Similarities, differences, and clinical implications. In A. Ie Christelle, T. Ngnoumen \& Ellen J. Langer (Eds.), The Wiley Blackwell handbook of mindfulness (pp. 48-57). Malden, Mass.: Wiley Blackwell. 
Carroll, J. E. (2004). On sustainability, religion, and ecology. Sustainability and spirituality (pp. 6-20). Albany, NY: SUNY Press.

Cerin, E., Barnett, A., \& Baranowski, T. (2009). Testing theories of dietary behavior change in youth using the mediating variable model with intervention programs. Journal of nutrition education and behavior, 41(5), 309-318.

Chairy, C. (2012). Spirituality, self-transcendence, and green purchase intention in college students. Journal of social and behavioral sciences, 57, 243-246.

Chen, Y., \& Moosmayer, D. C. (2020). When guilt is not enough: Interdependent selfconstrual as moderator of the relationship between guilt and ethical consumption in a Confucian context. Journal of Business Ethics, 161(3), 551-572.

Cherry, E. (2006). Veganism as a cultural movement: A relational approach. Social Movement Studies, 5(2), 155-170.

Cherry, E. (2015). I was a teenage vegan: Motivation and maintenance of lifestyle movements. Sociological Inquiry, 85(1), 55-74.

Chiesa, A. (2013). The difficulty of defining mindfulness: Current thought and critical issues. Mindfulness, 4(3), 255-268.

Coffey, K. A., Hartman, M., \& Fredrickson, B. L. (2010). Deconstructing mindfulness and constructing mental health: Understanding mindfulness and its mechanisms of action. Mindfulness, 1(4), 235-253.

Cole, M., \& Morgan, K. (2011). Vegaphobia: derogatory discourses of veganism and the reproduction of speciesism in UK national newspapers 1. The British Journal of Sociology, 62(1), 134-153.

Cornelissen, G., Pandelaere, M., Warlop, L., \& Dewitte, S. (2008). Positive cueing: Promoting sustainable consumer behavior by cueing common environmental behaviors as environmental. International Journal of Research in Marketing, 25(1), 46-55.

Conner, M., and Norman, P. (2005). Predicting health behaviour. Berkshire: McGrawHill Education.

De Jager Meezenbroek, E., Garssen, B., van den Berg, M., Van Dierendonck, D., Visser, A., \& Schaufeli, W. B. (2012a). Measuring spirituality as a universal human experience: A review of spirituality questionnaires. Journal of religion and health, 51(2), 336-354.

De Jager Meezenbroek, E., Garssen, B., van den Berg, M., Tuytel, G., van Dierendonck, D., Visser, A., \& Schaufeli, W. B. (2012b). Measuring spirituality as a universal human experience: Development of the Spiritual Attitude and Involvement List (SAIL). Journal of Psychosocial Oncology, 30(2), 141-167.

De La Sienra Servin, E. E., Smith, T., \& Mitchell, C. (2017). Worldviews, a mental construct hiding the potential of human behaviour: a new learning framework to 
guide education for sustainable development. The Journal of Sustainability Education.

Delle Fave, A., Massimini, F., \& Bassi, M. (2011). Psychological selection and optimal experience. In Psychological Selection and Optimal Experience Across Cultures (pp. 39-58). Springer, Dordrecht.

Delormier, T., Frohlich, K. L., \& Potvin, L. (2009). Food and eating as social practiceunderstanding eating patterns as social phenomena and implications for public health. Sociology of health \& illness, 31(2), 215-228.

Demetriou, A., \& Spanoudis, G. (2018). Growing minds: A developmental theory of intelligence, brain, and education. London: Routledge.

De Young, R. (1993). Changing behavior and making it stick: The conceptualization and management of conservation behavior. Environment and behavior, 25(3), 485505.

Dhandra, T. K. (2019). Achieving triple dividend through mindfulness: More sustainable consumption, less unsustainable consumption and more life satisfaction. Ecological economics, 161, 83-90.

Drewnowski, A. (2009). Obesity, diets, and social inequalities. Nutrition reviews, 67(1), 36-39.

Dreyfus, G. (2011). Is mindfulness present-centred and non-judgmental? A discussion of the cognitive dimensions of mindfulness. Contemporary Buddhism, 12(1), 41-54.

Ericson, T., Kjønstad, B. G., \& Barstad, A. (2014). Mindfulness and sustainability. Ecological Economics, 104, 73-79.

Evans, D., Welch, D., \& Swaffield, J. (2017). Constructing and mobilizing 'the consumer': Responsibility, consumption and the politics of sustainability. Environment and Planning A, 49(6), 1396-1412.

Fardet, A. (2016). Towards a more Holistic Vision of Human Nutrition to Prevent from Diet-Related Chronic diseases: the reductionist drift. International Journal of Food Science, Nutrition and Dietetics, 5(1e), 1-2.

Finger, J. D., Tylleskär, T., Lampert, T., and Mensink, G. B. (2013). Dietary behaviour and socioeconomic position: the role of physical activity patterns. PLoS One, 8(11), e78390.

Fischer, D., Stanszus, L., Geiger, S., Grossman, P., \& Schrader, U. (2017). Mindfulness and sustainable consumption: A systematic literature review of research approaches and findings. Journal of Cleaner Production, 162, 544-558.

Frank, P., Sundermann, A., \& Fischer, D. (2019). How mindfulness training cultivates introspection and competence development for sustainable consumption. International Journal of Sustainability in Higher Education. 
Gammel, S. (n.d.). Ethics and Morality. Basic ethical concepts. In: The Ethics PortfolioTechnical University Darmstadt for NanoCap. Retrieved June 26th, 2020 from: https://www.philosophie.tudarmstadt.de/media/philosophie_nanobuero/pdf_2/ethicsp ortfolio/ethics_moralitybwnewfont.pdf.

Garfield, A. M., Drwecki, B. B., Moore, C. F., Kortenkamp, K. V., \& Gracz, M. D. (2014). The Oneness Beliefs Scale: Connecting spirituality with pro-environmental behavior. Journal for the Scientific Study of Religion, 53(2), 356-372.

Geiger, S. M., Fischer, D., Schrader, U., \& Grossman, P. (2019). Meditating for the Planet: Effects of a Mindfulness-Based Intervention on Sustainable Consumption Behaviors. Environment and Behavior, 1-31.

Gethin, R. (2001). The Buddhist path to awakening: A study of the Bodhi-Pakkhiya Dhamma. Oxford: Oneworld Publications.

Gethin, R. (2011). On some definitions of mindfulness. Contemporary Buddhism, 12(1), 263-279.

Gethin, R. (2015). Buddhist conceptualizations of mindfulness. In K. W. Brown, J. D. Creswell, \& R. M. Ryan (Eds.), Handbook of mindfulness: Theory, research, and practice (pp. 9-41). New York: The Guilford Press.

Gifford, R. D., and Chen, A. K. (2017). Why aren't we taking action? Psychological barriers to climate-positive food choices. Climatic change, 140(2), 165-178.

Glotzbach, S., \& Baumgärtner, S. (2009). The relationship between intra-and intergenerational ecological justice: determinants of goal conflicts and synergies in sustainability policy (No. 141). Working Paper Series in Economics.

Grabovac, A. D., Lau, M. A., \& Willett, B. R. (2011). Mechanisms of mindfulness: A Buddhist psychological model. Mindfulness, 2(3), 154-166.

Greenberg, M. T., \& Mitra, J. L. (2015). From mindfulness to right mindfulness: The intersection of awareness and ethics. Mindfulness, 6(1), 74-78.

Grossmann, I. (2017). Wisdom in context. Perspectives on Psychological Science, 12(2), 233-257.

Grossman, P. (2008). On measuring mindfulness in psychosomatic and psychological research. Journal of psychosomatic research, 64(4), 405-408.

Grossman, P., \& Van Dam, N. T. (2011). Mindfulness, by any other name...: trials and tribulations of sati in western psychology and science. Contemporary Buddhism, 12(1), 219-239.

Grunert, K. G., Hieke, S., and Wills, J. (2014). Sustainability labels on food products: Consumer motivation, understanding and use. Food Policy, 44, 177-189.

Guagnano, G. A., Stern, P. C., \& Dietz, T. (1995). Influences on attitude-behavior relationships: A natural experiment with curbside recycling. Environment and behavior, 27(5), 699-718. 
Habing, B. (2003). Explanatory factor analysis. Department for Statistics at the University of South Carolina. Retrieved November 21st , 2018 from: http://people.stat.sc.edu/habing/ courses/530EFA.pdf.

Hall, P. A., \& Fong, G. T. (2007). Temporal self-regulation theory: A model for individual health behavior. Health Psychology Review, 1(1), 6-52.

Halme, M., Jasch, C., \& Scharp, M. (2004). Sustainable homeservices? Toward household services that enhance ecological, social and economic sustainability. Ecological economics, 51(1-2), 125-138.

Ham, C. B. (2000). The cultural challenge to individualism. Journal of Democracy, $11(1), 127-134$

Hamilton, S. (1996). Identity and experience: The constitution of the human being according to early Buddhism. London: Luzac Oriental.

Hanss, D., and Böhm, G. 2012. Sustainability seen from the perspective of consumers. International Journal of Consumer Studies 36(6): 678-687.

Harizan, S. H. M., \& Rahman, W. A. W. A. (2017). Spirituality of green purchase behavior: Does religious segmentation matter?. Journal of Research in Marketing, 6(3), 473-484.

Harvey, G. (2016). If 'Spiritual But Not Religious' People Are Not Religious What Difference Do They Make?. Journal for the Study of Spirituality, 6(2), 128-141.

Hawkins, N. A., Smith, T., Zhao, L., Rodriguez, J., Berkowitz, Z., \& Stein, K. D. (2010). Health-related behavior change after cancer: results of the American Cancer Society's studies of cancer survivors (SCS). Journal of cancer survivorship, 4(1), 20 32.

Hayes, S. C. (1984). Making sense of spirituality. Behaviorism, 99-110.

Hayes, S. C. (Ed.). (1994). Acceptance and change: Content and context in psychotherapy. Reno, NV: Context Press.

Hayton, J. C., Allen, D. G., \& Scarpello, V. (2004). Factor retention decisions in exploratory factor analysis: A tutorial on parallel analysis. Organizational research methods, 7(2), 191-205.

He, H. A., \& Greenberg, S. (2008). Motivating sustainable energy consumption in the home. University of Calgary. Canada.

Hedenus, F., Wirsenius, S., \& Johansson, D. J. (2014). The importance of reduced meat and dairy consumption for meeting stringent climate change targets. Climatic change, 124(1-2), 79-91.

Hedlund-de Witt, A. (2013). Worldviews and their significance for the global sustainable development debate. Environmental Ethics, 35(2), 133-162. 
Hedlund-de Witt, A., De Boer, J., \& Boersema, J. J. (2014). Exploring inner and outer worlds: A quantitative study of worldviews, environmental attitudes, and sustainable lifestyles. Journal of Environmental Psychology, 37, 40-54.

Heelas, P., Woodhead, L., Seel, B., Szerszynski, B., \& Tusting, K. (2005). The spiritual revolution: Why religion is giving way to spirituality (p. 2). Oxford: Blackwell.

Heiervang, E., \& Goodman, R. (2011). Advantages and limitations of web-based surveys: evidence from a child mental health survey. Social psychiatry and psychiatric epidemiology, 46(1), 69-76.

Heintzman, P. (2009). Nature-based recreation and spirituality: A complex relationship. Leisure Sciences, 32(1), 72-89.

Hemforth, B., \& Konieczny, L. (2006). Language processing: construction of mental models or more?. In C. Held, M. Knauff, \& G. Vosgerau (Eds.). Advances in Psychology (Vol. 138, pp. 189-204). Amsterdam, Boston: Elsevier.

Hendrickson, K. L., \& Rasmussen, E. B. (2013). Effects of mindful eating training on delay and probability discounting for food and money in obese and healthy-weight individuals. Behaviour research and therapy, 51(7), 399-409.

Heuser, A., Thomsen, B., Wilhelm, B., Rocha, C., ... \& Sachs, W. (2016) Better and different! Transforming food systems through agroecology. Achen, Berlin: INKOTA, Oxfam, and MISEREOR. Retrieved June 6th, 2020 from: https://www.misereor.de/fileadmin/publikationen/broschuere-agraroekologie.pdf.

Hill, P. C., Pargament, K. I., Hood, R. W., McCullough, J. M. E., Swyers, J. P., Larson, D. B., \& Zinnbauer, B. J. (2000). Conceptualizing religion and spirituality: Points of commonality, points of departure. Journal for the theory of social behaviour, 30(1), 51-77.

Horlings, L. G. (2015). The inner dimension of sustainability: personal and cultural values. Current Opinion in Environmental Sustainability, 14, 163-169.

Hunecke, M., \& Richter, N. (2019). Mindfulness, construction of meaning, and sustainable food consumption. Mindfulness, 10(3), 446-458.

Husemann, K. C., \& Eckhardt, G. M. (2019). Consumer spirituality. Journal of marketing management, 35(5-6), 391-406.

Insight. (n.d.) In: Cambridge Dictionary Online. Cambridge Dictionary. Retrieved June 5th, 2020 from: https://dictionary.cambridge.org/de/worterbuch/englisch/insight.

Isaac, K. S., Hay, J. L., \& Lubetkin, E. I. (2016). Incorporating spirituality in primary care. Journal of religion and health, 55(3), 1065-1077.

Jacob, J., Jovic, E., \& Brinkerhoff, M. B. (2009). Personal and planetary well-being: Mindfulness meditation, pro-environmental behavior and personal quality of life in a survey from the social justice and ecological sustainability movement. Social Indicators Research, 93(2), 275-294. 
Joffe, H. (2008). The power of visual material: Persuasion, emotion and identification. Diogenes, 55(1), 84-93.

Johnson, C., Burke, C., Brinkman, S., \& Wade, T. (2017). Development and validation of a multifactor mindfulness scale in youth: The Comprehensive Inventory of Mindfulness Experiences-Adolescents (CHIME-A). Psychological assessment, 29(3), 264.

Johnson, R. K. (2002). Dietary intake-how do we measure what people are really eating? Obesity, 10(s11), 63S-68S.

Jordan, C. H., Wang, W., Donatoni, L., \& Meier, B. P. (2014). Mindful eating: Trait and state mindfulness predict healthier eating behavior. Personality and Individual differences, 68, 107-111.

Joshi, Y. (2017). Investigating the influence of spirituality, environmental concern and ecological knowledge on consumers' green purchase intention. PURUSHARTHA-A journal of Management, Ethics and Spirituality, 9(2), 54-61.

Kabat-Zinn, J. (2000). Indra's net at work: The mainstreaming of Dharma practice in society. In G. Watson, S. Batchelor, \& G. Claxton. The Psychology of Awakening: Buddhism, science and our day-to-day lives (pp. 225-250). York Beach: Rider.

Kabat-Zinn, J. (2014). Wherever you go, there you are: Mindfulness meditation in everyday life. New York: Hachette Books.

Kale, S. H. (2004). Spirituality, religion, and globalization. Journal of Macromarketing, 24(2), 92-107.

Kalupahana, D. J. (1995). Ethics in early Buddhism. Delhi: Motilal Banarsidass Publishing.

Kang, C., \& Whittingham, K. (2010). Mindfulness: A dialogue between Buddhism and clinical psychology. Mindfulness, 1(3), 161-173.

Katz, C., \& McPherson, T. (2019). Veganism as a food ethic. In H. Meiselman (Ed.), Handbook of eating and drinking. Dordrecht: Springer.

Kearney, D. J., Milton, M. L., Malte, C. A., McDermott, K. A., Martinez, M., \& Simpson, T. L. (2012). Participation in mindfulness-based stress reduction is not associated with reductions in emotional eating or uncontrolled eating. Nutrition research, 32(6), 413-420.

Kiken, L. G., Garland, E. L., Bluth, K., Palsson, O. S., \& Gaylord, S. A. (2015). From a state to a trait: Trajectories of state mindfulness in meditation during intervention predict changes in trait mindfulness. Personality and individual differences, 81, 4146 .

Kinnear, T. C., Taylor, J. R., \& Ahmed, S. A. (1974). Ecologically concerned consumers: who are they? Ecologically concerned consumers can be identified. Journal of marketing, 38(2), 20-24. 
Klein, E. (2017). The World Bank on mind, behaviour and society. Development and Change, 48(3), 481-501.

Kormos, C., \& Gifford, R. (2014). The validity of self-report measures of proenvironmental behavior: A meta-analytic review. Journal of Environmental Psychology, 40, 359-371.

Kropp, C. (2018). Urban food movements and their transformative capacities. The International Journal of Sociology of Agriculture and Food, 24(3), 413-430.

Kuan, T. F. (2008). Mindfulness in early Buddhism. New approaches through psychology and textual analysis of Pali, Chinese and Sanskrit sources. London: Routledge.

Kuan, T. F. (2012). Cognitive operations in Buddhist meditation: Interface with Western psychology. Contemporary Buddhism, 13(1), 35-60.

Landwirtschaftskammer. (n.d.). Solidarische Landwirtschaft (SoLaWi). Retrieved June 6th, 2020

from: https://www.landwirtschaftskammer.de/landwirtschaft/landentwicklung/urban/mitmac hen/informieren/solawi.htm.

Langer, E. J. (1989). Minding matters: The consequences of mindlessnessmindfulness. In L. Berkowitz (Ed.). Advances in experimental social psychology (Vol. 22, pp. 137-173). Cambridge: Academic Press.

Lee, J. D., Bahl, A., Black, G. S., Duber-Smith, D. C., \& Vowles, N. S. (2016). Sustainable and non-sustainable consumer behavior in young adults. Young Consumers 17(1), 78-93.

Lee, M. T. (2015). North Central Sociological Association presidential address. The mindful society: Contemplative sociology, meta-mindfulness, and human flourishing. Sociological Focus, 48(4), 271-299.

Leigh, J., Bowen, S., \& Marlatt, G. A. (2005). Spirituality, mindfulness and substance abuse. Addictive behaviors, 30(7), 1335-1341.

Leitzmann, C., Keller, M., and Hahn, A. (2005). Alternative Ernährungsformen. Stuttgart: Hippokrates Verlag.

Li, C. (2013). Little's test of missing completely at random. The Stata Journal, 13(4), 795-809.

Liang, J. J. C. (2009). The process of decentering: a phenomenological study of Asian American Buddhists from the Fo Guan Shan Temple Buddhist order (Doctoral dissertation, University of Missouri--Columbia).

Lim, W. M. (2017). Inside the sustainable consumption theoretical toolbox: Critical concepts for sustainability, consumption, and marketing. Journal of Business Research, 78, 69-80. 
Lu, L. C., Chang, H. H., \& Chang, A. (2015). Consumer personality and green buying intention: The mediate role of consumer ethical beliefs. Journal of Business Ethics, 127(1), 205-219.

Markle, G. L. (2013). Pro-environmental behavior: does it matter how it's measured? Development and validation of the pro-environmental behavior scale (PEBS). Human ecology, 41(6), 905-914.

Marlatt, G. A., \& Kristeller, J. L. (2003). Mindfulness and meditation. In W. R. Miller (Ed.): Integrating spirituality into treatment. Resources for practitioners (pp. 67-84). Washington, DC: American Psychological Association.

Marmor-Lavie, G., \& Stout, P. A. (2016). Consumers' insights about spirituality in advertising. Journal of Media and Religion, 15(4), 169-185.

Maskarinec, G., Murphy, S., Shumay, D. M., \& Kakai, H. (2001). Dietary changes among cancer survivors. European journal of cancer care, 10(1), 12-20.

Mason, A. E., Epel, E. S., Kristeller, J., Moran, P. J., Dallman, M., Lustig, R. H., ... \& Daubenmier, J. (2016). Effects of a mindfulness-based intervention on mindful eating, sweets consumption, and fasting glucose levels in obese adults: data from the SHINE randomized controlled trial. Journal of behavioral medicine, 39(2), 201213.

Mason, P., \& Lang, T. (2017). Sustainable diets: how ecological nutrition can transform consumption and the food system. New York: Taylor \& Francis.

Mathieu, J.( 2009). What should you know about mindful and intuitive eating?. Journal of the Academy of Nutrition and Dietetics, 109(12), 1982-1987.

Matko, K., \& Sedlmeier, P. (2019). What is Meditation? Proposing an EmpiricallyDerived Classification System. Frontiers in psychology, 10, 2276.

McConnon, A., Fletcher, P. L., Cade, J. E., Greenwood, D. C., \& Pearman, A. D. (2004). Differences in perceptions of functional foods: UK public vs. nutritionists. Nutrition Bulletin, 29(1), 11-18.

McGhee, P., \& Grant, P. (2008). Spirituality and ethical behaviour in the workplace: Wishful thinking or authentic reality. EJBO-Electronic Journal of Business Ethics and Organization Studies, 13(2), 61-69.

McKenzie-Mohr, D., \& Oskamp, S. (1995). Psychology and sustainability: An introduction. Journal of Social Issues, 51(4), 1-14.

McLeod, S. A. (2016). Id, ego and superego. Simply Psychology, 3, 1-4.

Meybeck, A., \& Gitz, V. (2017). Sustainable diets within sustainable food systems. Proceedings of the Nutrition Society, 76(1), 1-11.

Mihas, P. (2019). Qualitative data analysis. In G.W. Noblit (Ed.): Oxford research encyclopedia of education. Oxford: Oxford University Press. 
Minton, E. A., Kahle, L. R., \& Kim, C. H. (2015). Religion and motives for sustainable behaviors: A cross-cultural comparison and contrast. Journal of Business Research, 68(9), 1937-1944.

Monteiro, L. M., Musten, R. F., \& Compson, J. (2015). Traditional and contemporary mindfulness: finding the middle path in the tangle of concerns. Mindfulness, 6(1), 113.

Muller-Eie D. \& Bjorno L. (2016). Urban sustainability and individual behavior. In C.A. Brebbia (Ed.): Sustainable Development (pp. 29-40). Southhampton, UK: WIT Press.

Nagel, T. (1974). What is it like to be a bat?. The philosophical review, 83(4), 435-450.

National Research Council (US). (1982) Committee on Nutrition of the Mother, \& Preschool Child. Alternative dietary practices and nutritional abuses in pregnancy: Summary report. Washington, DC: National Academy press.

Office of the Registrar General \& Census Commissioner. (2001). Age structure and marital status in India. Retrieved June 15th, 2019 from: http://censusindia.gov.in/Census_And_You/age_structure_and_marital_status.aspx

O'Guinn, T. C., \& Belk, R. W. (1989). Heaven on earth: consumption at Heritage Village, USA. Journal of Consumer Research, 16(2), 227-238.

Olsen Marvin, E., Lodwick, D. G., \& Dunlap, R. E. (1992). Viewing the World Ecologically. Boulder, CO: Westview Press.

Omvedt, G. (2003). Buddhism in India: challenging Brahmanism and caste. New Delhi: Sage Publications India.

Orr, D. W. (2002). Four challenges of sustainability. Conservation biology, 16(6), 14571460.

Osborne, J. W. (2015). What is rotating in exploratory factor analysis?. Practical Assessment, Research, and Evaluation, 20(1), 2.

Panno, A., Giacomantonio, M., Carrus, G., Maricchiolo, F., Pirchio, S., \& Mannetti, L. (2018). Mindfulness, pro-environmental behavior, and belief in climate change: the mediating role of social dominance. Environment and Behavior, 50(8), 864-888.

Pargament, K. I. (1999). The psychology of religion and spirituality? Yes and no. The international journal for the psychology of religion, 9(1), 3-16.

Parodi, O., \& Tamm, K. (Eds.). (2018). Personal sustainability: exploring the far side of sustainable development. London: Routledge.

Pawlik V. (2020a). Anzahl der Personen in Deutschland, die sich selbst als Vegetarier einordnen oder als Leute, die weitgehend auf Fleisch verzichten*, von 2014 bis 2019.

online: https://de.statista.com/statistik/daten/studie/173636/umfrage/lebens einstellunganzahl-vegetarier/. (06.06.2020). 
Pawlik V. (2020b). Personen in Deutschland, die sich selbst als Veganer einordnen oder als Leute, die weitgehend auf tierische Produkte verzichten, in den Jahren 2016 bis 2020.2 Available online: https://de.statista.com/statistik/daten/studie/445155/umfrage /umfrage-indeutschland-zur-anzahl-der-veganer/. (21.10.2020).

Payutto, P. (1988). Sammasati: An exposition of right mindfulness. Bangkok: Buddhadhamma Foundation.

Pelletier, L. G., Lavergne, K. J., \& Sharp, E. C. (2008). Environmental psychology and sustainability: Comments on topics important for our future. Canadian Psychology/psychologie canadienne, 49(4), 304.

Pelletier, N., \& Tyedmers, P. (2010). Forecasting potential global environmental costs of livestock production 2000-2050. Proceedings of the National Academy of Sciences, 107(43), 18371-18374.

Percy, W. H., Kostere, K., \& Kostere, S. (2015). Generic qualitative research in psychology. The Qualitative Report, 20(2), 76-85.

Pérez-Sales, P., Eiroa-Orosa, F. J., Olivos, P., Barbero-Val, E., Fernández-Liria, A., \& Vergara, M. (2012). Vivo Questionnaire: A measure of human worldviews and identity in trauma, crisis, and loss-validation and preliminary findings. Journal of Loss and Trauma, 17(3), 236-259.

Phan, U. T., \& Chambers IV, E. (2016). Motivations for choosing various food groups based on individual foods. Appetite, 105, 204-211.

Pietrzyk, K., (2006). "Freeganism: food for Mind, Body and Soul". Available online: http://londoncommons.ca/node/594 (25.10.2020)

Pollard, J., Kirk, S. L., \& Cade, J. E. (2002). Factors affecting food choice in relation to fruit and vegetable intake: a review. Nutrition research reviews, 15(2), 373-387.

Powell, A. (2001). Spirituality and science: a personal view. Advances in Psychiatric Treatment, 7(5), 319-321.

Power, K., \& Mont, O. (2010). The role of formal and informal forces in shaping consumption and implications for sustainable society: Part II. Sustainability, 2(8), 2573-2592.

Purser, R. (2019). McMindfulness: How mindfulness became the new capitalist spirituality. London: Watkins Media Limited.

Purser, R. E., \& Milillo, J. (2015). Mindfulness revisited: A Buddhist-based conceptualization. Journal of Management Inquiry, 24(1), 3-24.

Queen, C. S., \& King, S. B. (Eds.). (1996). Engaged buddhism: buddhist liberation movements in Asia. Albany, NY: State Univ. of New York Press. 
Rahman, M. S. (2012). Young consumer's perception on foreign made fast moving consumer goods: the role of religiosity, spirituality and animosity. International Journal of Business and Management Science, 5(2), 103-118.

Redden, G. (2016). Revisiting the spiritual supermarket: Does the commodification of spirituality necessarily devalue it?. Culture and Religion, 17(2), 231-249.

Reisch, L., Eberle, U., \& Lorek, S. (2013). Sustainable food consumption: an overview of contemporary issues and policies. Sustainability: Science, Practice and Policy, 9(2), 7-25.

Renner, B., Sproesser, G., Strohbach, S., \& Schupp, H. T. (2012). Why we eat what we eat. The Eating Motivation Survey (TEMS). Appetite, 59(1), 117-128.

Resnicow, K., \& Vaughan, R. (2006). A chaotic view of behavior change: a quantum leap for health promotion. International Journal of Behavioral Nutrition and Physical Activity, 3(1), 25.

Rhys Davids, T. W. \& Rhys Davids, C. A. (1966-1971): Dialogues of the Buddha. London: Luzac.

Rice, R. E. (2006). Enhancing the Quality of Teaching and Learning: The US Experience. New Directions for Higher Education, 133, 13-22.

Rinallo, D., Borghini, S., Bamossy, G., and Kozinets, R. V. (2013). When sacred objects go $\mathrm{B} \AA \mathrm{a}(\mathrm{n}) \mathrm{d}$ : Fashion rosaries and the contemporary linkage of religion and commerciality. In D. Rinallo, L. Scott, and P. Maclaran (Eds.), Spirituality and consumption (pp. 29-40). New York: Routledge.

Rodriguez-Rad, C. J., \& Ramos-Hidalgo, E. (2018). Spirituality, consumer ethics, and sustainability: the mediating role of moral identity. Journal of consumer marketing.

Rosenberg, E. L. (2004). Mindfulness and consumerism. In T. Kasser \& A. de Kanner (Eds), Psychology and consumer culture. The struggle for a good life in a materialistic world (pp. 107-125). Washington, DC: American Psychological Association.

Rosenfeld, D. L. (2019). Why some choose the vegetarian option: Are all ethical motivations the same?. Motivation and Emotion, 43(3), 400-411.

Royston, P. (2009). Multiple imputation of missing values: further update of ice, with an emphasis on categorical variables. The Stata Journal, 9(3), 466-477.

Sahdra, B. K., Shaver, P. R., \& Brown, K. W. (2010). A scale to measure nonattachment: A Buddhist complement to Western research on attachment and adaptive functioning. Journal of personality assessment, 92(2), 116-127.

Sainsbury, K., Halmos, E. P., Knowles, S., Mullan, B., \& Tye-Din, J. A. (2018). Maintenance of a gluten free diet in coeliac disease: The roles of self-regulation, habit, psychological resources, motivation, support, and goal priority. Appetite, 125, 356-366. 
Sangharakshita. U. (2003). Living with awareness: A guide to the Satipațhāna Sutta. Birmingham: Windhorse Publications.

Sanne, C. (2002). Willing consumers-or locked-in? Policies for a sustainable consumption. Ecological economics, 42(1-2), 273-287.

Sauer, S., Walach, H., Schmidt, S., Hinterberger, T., Lynch, S., Büssing, A., \& Kohls, N. (2013). Assessment of mindfulness: Review on state of the art. Mindfulness, 4(1), 3-17.

Saxena K. (2019). Buddhist ethical approach to sustainable societies. In: T.D. Thien, \& T.N. Tu (Eds.), Buddhist approach to global education in ethics. (pp. 125-137). Hanoi: Hong Duc Publishing House.

Schipper, J. (2012). Toward a Buddhist sociology: theories, methods, and possibilities. The American Sociologist, 43(2), 203-222.

Schmidt, S. (2011). Mindfulness in east and west-is it the same?. In H. Walach, S. Schmidt, \& W. B. Jonas (Eds.), Neuroscience, consciousness and spirituality (pp. 23-38). Dordrecht: Springer.

Schmitt, T. A. (2011). Current methodological considerations in exploratory and confirmatory factor analysis. Journal of Psychoeducational Assessment, 29(4), 304321.

Schumacher, E. F. (1995). A Guide for the Perplexed. Gütersloh: Random House.

Schwartz, S. H. (1977). Normative influences on altruism. In L. Berkowitz (Ed.), Advances in experimental social psychology (10 vol. 1, pp. 221-279) New York: Academic $P$.

Segal, Z. V., Teasdale, J. D., Williams, J. M., \& Gemar, M. C. (2002). The mindfulnessbased cognitive therapy adherence scale: Inter-rater reliability, adherence to protocol and treatment distinctiveness. Clinical Psychology \& Psychotherapy, 9(2), 131-138.

Shapiro, S. L. (2009). The integration of mindfulness and psychology. Journal of clinical psychology, 65(6), 555-560.

Sharma, C. S., \& Sharma, N. (2017). Relationship between consumers' spirituality and green purchasing intentions: The mediation effect of perceived consumer effectiveness. IIM Kozhikode Society \& Management Review, 6(2), 204-214.

Shwom, R., \& Lorenzen, J. A. (2012). Changing household consumption to address climate change: social scientific insights and challenges. Wiley Interdisciplinary Reviews: Climate Change, 3(5), 379-395.

Simon, H. A. (1955). A behavioral model of rational choice. The quarterly journal of economics, 69(1), 99-118.

Soron, D. (2010). Sustainability, self-identity and the sociology of consumption. Sustainable development, 18(3), 172-181. 
Spaargaren, G., Oosterveer, P., \& Loeber, A. (Eds.). (2013). Food practices in transition: changing food consumption, retail and production in the age of reflexive modernity. New York: Routledge.

Spiller, A., Zühlsdorf, A., \& Nitzko, S. (2017). Instrumente der Ernährungspolitik. Ein Forschungs-überblick - Teil 2. Ernährungs Umschau, 64(4), 204-209.

Stanszus, L. S., Frank, P., \& Geiger, S. M. (2019). Healthy eating and sustainable nutrition through mindfulness? Mixed method results of a controlled intervention study. Appetite, 141, 104325.

Statista (2015). Distribution of average monthly income in households across India in 2015. Retrieved June 15th, 2019 from: https://www.statista.com/statistics/653897/average-monthly-household-incomeindia/.

Statista, (2020). Anzahl der Personen in Deutschland, die sich selbst als Vegetarier einordnen oder als Leute, die weitgehend auf Fleisch verzichten*, von 2014 bis 2019. Retrieved June 6th, 2020 from: https://de.statista.com/statistik/daten/studie/173636/umfrage/lebenseinstellunganzahlvegetarier/.

Statisticalatlas (2018). Overview of the United States. Retrieved June 15th, 2019 from: https://statisticalatlas.com/United-States/Overview.

Stern, P. C. (2000). New environmental theories: toward a coherent theory of environmentally significant behavior. Journal of social issues, 56(3), 407-424.

Stern, P. C., Dietz, T., Abel, T., Guagnano, G. A., \& Kalof, L. (1999). A value-beliefnorm theory of support for social movements: The case of environmentalism. Human ecology review, 81-97.

Story, M., Kaphingst, K. M., Robinson-O'Brien, R., \& Glanz, K. (2008). Creating healthy food and eating environments: policy and environmental approaches. Annual revisions in public health, 29, 253-272.

Subrahmanyan, S., \& Gould, S. (2012). Achieving sustainable consumption through spiritual practices. PURUSHARTHA-A journal of Management, Ethics and Spirituality, 5(2), 79-91.

Tahara, Y. and Shibata, S. 2013. Chronobiology and nutrition. Neuroscience, 253, 7888.

Tang, Y. Y. (2017). Mindfulness Meditation and Behavior Change. In Y. Y. Tang (Ed.), The neuroscience of mindfulness meditation (pp. 35-44). Cham: Palgrave Macmillan.

Tang, Y., Geng, L., Schultz, P. W., Zhou, K., \& Xiang, P. (2017). The effects of mindful learning on pro-environmental behavior: A self-expansion perspective. Consciousness and Cognition, 51, 140-148. 
Tang, Y. Y., Hölzel, B. K., \& Posner, M. I. (2015). The neuroscience of mindfulness meditation. Nature Reviews Neuroscience, 16(4), 213-225.

Tapper, K., Shaw, C., Ilsley, J., Hill, A. J., Bond, F. W., \& Moore, L. (2009). Exploratory randomised controlled trial of a mindfulness-based weight loss intervention for women. Appetite, 52(2), 396-404.

Teddlie, C., \& Tashakkori, A. (2009). Foundations of mixed methods research: Integrating quantitative and qualitative approaches in the social and behavioral sciences. Thousand Oaks, CA: Sage.

Tenenbaum, J. B., Kemp, C., Griffiths, T. L., \& Goodman, N. D. (2011). How to grow a mind: Statistics, structure, and abstraction. Science, 331(6022), 1279-1285.

Thera, N. (2005). The heart of buddhist meditation: Satipațthāna: A handbook of mental training based on the Buddha's way of mindfulness, with an anthology of relevant texts translated from the Pali and Sanskrit. Kandy: Buddhist Publication Society.

Thomas, E. J. (1929). The Path of Purity. Being a translation of Buddhaghosa's Visuddhimagga. By Pe Maung Tin. Part II. Journal of the Royal Asiatic Society, 61(3), 614-615.

Tischler, L. (1999). The growing interest in spirituality in business. Journal of Organizational Change Management.

Tracy, S. J. (2019). Qualitative research methods: Collecting evidence, crafting analysis, communicating impact. Hoboken, NJ: John Wiley \& Sons.

Ulluwishewa, R. (2014). Spirituality and sustainable development. London: Palgrave Macmillan.

Vagh, M. G. 2019. Influence and intimation of yogic diet over the mind. Journal of Yogic, Human Movement and Sport Science 4(1): 878-881.

Vago, D. R., Gupta, R., \& Lazar, S. (2018). How does mindfulness affect cognition? A reflection on confounding issues surrounding mindfulness-based intervention research targeting cognitive outcomes. Current Opinion in Psychology, 28, 143-150.

Vandenberghe, L., \& Costa Prado, F. (2009). Law and grace in Saint Augustine: a fresh perspective on mindfulness and spirituality in behaviour therapy. Mental Health, Religion and Culture, 12(6), 587-600.

Van Egmond, N. D., \& De Vries, H. J. M. (2011). Sustainability: The search for the integral worldview. Futures, 43(8), 853-867.

Vaterlaus, J. M., Patten, E. V., Roche, C., \& Young, J. A. (2015). \# Gettinghealthy: The perceived influence of social media on young adult health behaviors. Computers in Human Behavior, 45, 151-157.

Vaughan, F. (2002). What is spiritual intelligence?. Journal of humanistic psychology, 42(2), 16-33. 
Velez de Cea J.A. (2019) Teaching Buddhist ethics through the life of the Buddha. In: T.D. Thien, \& T.N. Tu (Eds.), Buddhist approach to global education in ethics. (pp. 19-40). Hanoi: Hong Duc Publishing House.

Ver Beek, K. A. (2000). Spirituality: A development taboo. Development in practice, 10(1), 31-43.

Verdugo, V. C. (2012). The positive psychology of sustainability. Environment, Development and Sustainability, 14(5), 651-666.

Vermeir, I., \& Verbeke, W. (2006). Sustainable food consumption: Exploring the consumer "attitude-behavioral intention" gap. Journal of Agricultural and Environmental ethics, 19(2), 169-194.

Wamsler, C., Brossmann, J., Hendersson, H., Kristjansdottir, R., McDonald, C., \& Scarampi, P. (2018). Mindfulness in sustainability science, practice, and teaching. Sustainability Science, 13(1), 143-162.

Werhane, P. H., Hartman, L. P., Moberg, D., Englehardt, E., Pritchard, M., \& Parmar, B. (2011). Social constructivism, mental models, and problems of obedience. Journal of business ethics, 100(1), 103-118.

Werner, A., Spiller, A., \& Meyerding, S. G. (2020). The yoga of sustainable diets: Exploring consumers mind and spirit. Journal of Cleaner Production, 243, 118473.

Wilber, K. (2004). Integral psychology: Consciousness, spirit, psychology, therapy. Nova Religio: The Journal of Alternative and Emergent Religions, 8(2), 125-127.

Willett, W., Rockström, J., Loken, B., Springmann, M., Lang, T., Vermeulen, S., ... \& Jonell, M. (2019). Food in the Anthropocene: the EAT-Lancet Commission on healthy diets from sustainable food systems. The Lancet, 393(10170), 447-492.

Williams, J. M. G. (2010). Mindfulness and psychological process. Emotion, 10(1), 1.

Williams, J. M. G., \& Kabat-Zinn, J. (2011). Mindfulness: diverse perspectives on its meaning, origins, and multiple applications at the intersection of science and dharma. Contemporary Buddhism, 12(1), 1-18.

Whitmarsh, S. (2013). Nonreactivity and metacognition in mindfulness (Doctoral dissertation). Donders Graduate School for Cognitive Neuroscience series (120).

Zelazo, P. D., \& Lyons, K. E. (2012). The potential benefits of mindfulness training in early childhood: A developmental social cognitive neuroscience perspective. Child Development Perspectives, 6(2), 154-160.

Zepeda, L., \& Deal, D. (2009). Organic and local food consumer behaviour: Alphabet theory. International Journal of Consumer Studies, 33(6), 697-705.

Zinnbauer, B. J., Pargament, K. I., Cole, B., Rye, M. S., Butter, E. M., Belavich, T. G., ... \& Kadar, J. L. (1997). Religion and spirituality: Unfuzzying the fuzzy. Journal for the scientific study of religion, 549-564. 


\section{Authors Contributions}

The table below summarizes the scientific contributions to the essays presented within the framework of this dissertation. The contributors are set to order according to their investment into the project.

\begin{tabular}{|c|c|c|c|}
\hline Contribution & Essay 1 & Essay 2 & Essay 3 \\
\hline Conceptualization & AW; Dr. Antje Risius & AW; Prof. Achim. Spiller & AW \\
\hline Creating research design & AW; Dr. Antje Risius & AW; Prof. Stephan Meyerding & AW \\
\hline $\begin{array}{l}\text { Instrument construction and } \\
\text { questionnaire design }\end{array}$ & AW; Dr. Antje Risius & AW; Prof. Achim Spiller & AW \\
\hline Data analysis & AW & AW; Dr. Alexander Silbersdorff & - \\
\hline Transcription of data & Digitalmeister GmbH; AW & - & - \\
\hline $\begin{array}{l}\text { Interpretation of statistical } \\
\text { analysis }\end{array}$ & AW; Dr. Antje Risius & $\begin{array}{l}\text { AW; Dr. Jennifer Lorenz; Prof. Achim } \\
\text { Spiller }\end{array}$ & - \\
\hline $\begin{array}{l}\text { Interpretation of theoretical } \\
\text { material }\end{array}$ & - & - & $\begin{array}{l}\text { AW; Prof. Rupert Gethin; Prof. } \\
\text { Mahesh Deokar }\end{array}$ \\
\hline Proofreading & Merindah Loessl & Hauptstadtstudio Freier Lektoren & Hauptstadtstudio Freier Lektoren \\
\hline
\end{tabular}

AW = Aspasia Werner 


\section{Declaration}

(1) I, hereby, declare that this dissertation has not been presented to any other examining body either in its present or a similar form.

Furthermore, I also affirm that I have not applied for a Ph.D. at any other higher school of education.

Goettingen, 10.08.2020

(Signature)

...ASPASIA WERNER

(Name in block capitals)

(2) I, hereby, solemnly declare that this dissertation was undertaken independently and without any unauthorized aid.

Goettingen, 10.08.2020

(Signature)

ASPASIA WERNER

(Name in block capitals) 